\title{
Dynamic Optimization Algorithms for Baseload Power Plant Cycling under Variable Renewable Energy
}

\author{
Rebecca Hyen Hae Kim \\ West Virginia University, rhkim@mix.wvu.edu
}

Follow this and additional works at: https://researchrepository.wvu.edu/etd

Part of the Process Control and Systems Commons

\section{Recommended Citation}

Kim, Rebecca Hyen Hae, "Dynamic Optimization Algorithms for Baseload Power Plant Cycling under Variable Renewable Energy" (2021). Graduate Theses, Dissertations, and Problem Reports. 8101. https://researchrepository.wvu.edu/etd/8101

This Dissertation is protected by copyright and/or related rights. It has been brought to you by the The Research Repository @ WVU with permission from the rights-holder(s). You are free to use this Dissertation in any way that is permitted by the copyright and related rights legislation that applies to your use. For other uses you must obtain permission from the rights-holder(s) directly, unless additional rights are indicated by a Creative Commons license in the record and/ or on the work itself. This Dissertation has been accepted for inclusion in WVU Graduate Theses, Dissertations, and Problem Reports collection by an authorized administrator of The Research Repository @ WVU.

For more information, please contact researchrepository@mail.wvu.edu. 


\title{
Dynamic Optimization Algorithms for Baseload Power Plant Cycling under Variable Renewable Energy
}

\author{
Rebecca Hyen Hae Kim \\ Dissertation submitted to the \\ Benjamin M. Statler College of Engineering and Mineral Resources \\ at West Virginia University
}

In partial fulfillment of the requirements for the degree of

\author{
Doctor of Philosophy \\ in \\ Chemical Engineering \\ Fernando V. Lima, PhD, Chair \\ Debangsu Bhattacharyya, PhD \\ Richard Turton, PhD \\ Stephen Zitney, PhD \\ Jignesh Solanki, PhD \\ Urmila Diwekar, PhD \\ Department of Chemical and Biomedical Engineering \\ Morgantown, West Virginia \\ 2021
}

Keywords: dynamic real-time optimization, multi-objective optimization, particle swarm optimization, baseload power plant cycling

Copyright 2021 Rebecca Hyen Hae Kim 


\title{
Abstract
}

\section{Dynamic Optimization Algorithms for Baseload Power Plant Cycling under Variable Renewable Energy}

\author{
Rebecca Kim
}

The growing deployment of variable renewable energy (VRE) sources, such as wind and solar, is mainly due to the decline in the cost of renewable technologies and the increase of societal and cultural pressures. Solar and wind power generation are also known to have zero marginal costs and fuel emissions during dispatch. Thereby, the VRE from these sources should be prioritized when available. However, the rapid deployment of VRE has heightened concerns regarding the challenges in the integration between fossilfueled and renewable energy systems. The high variability introduced by the VRE as well as the limited alignment between demand and wind/solar power generation led to the increased need of dispatchable energy sources such as baseload natural gas- and coalfired power plants to cycle their power outputs more often to reliably supply the net load. The increasing power plant cycling can introduce unexpected inefficiencies into the system that potentially incur higher costs, emissions, and wear-and-tear, as the power plants are no longer operating at their optimal design points.

In this dissertation, dynamic optimization algorithms are developed and implemented for baseload power plant cycling under VRE penetration. Specifically, two different dynamic optimization strategies are developed for the minute and hourly time scales of grid operation. The minute-level strategy is based on a mixed-integer linear programming (MILP) formulation for dynamic dispatch of energy systems, such as natural gas- and coal-fired power plants and sodium sulfur batteries, under VRE while considering power plant equipment health-related constraints. The hourly-level strategy is based on a Nonlinear Multi-objective dynamic real-time Predictive Optimization (NMPO) implemented in a supercritical pulverized coal-fired (SCPC) power plant with a postcombustion carbon capture system (CCS), considering economic and environmental objectives. Different strategies are employed and explored to improve computational 
tractability, such as mathematical reformulations, automatic differentiation (AD), and parallelization of a metaheuristic particle swarm optimization (PSO) component.

The MILP-based dynamic dispatch framework is used to simulate case studies considering different loads and renewable penetration levels for a suite of energy systems. The results show that grid flexibility is mostly provided by the natural gas power plant, while the batteries are used sparingly. Additionally, considering the post-optimization equivalent carbon analysis, the environmental performance is intrinsically connected to grid flexibility and the level of VRE penetration. The stress results reinforce the necessity of further considering and including equipment health-related constraints during dispatch.

The results of the NMPO successfully implemented for a large-scale SCPC-CCS show that the optimal compromise is automatically chosen from the Pareto front according to a set of weights for the objectives with minimal interaction between the framework and the decision maker. They also indicate that to setup the optimization thresholds and constraints, knowledge of the power system operations is essential. Finally, the market and carbon policies have an impact on the optimal compromise between the economic and environmental objectives. 


\section{Dedication}

\section{Dedicated to}

\section{God,}

my parents Kyung and Dong,

my sisters Esther and Hanna,

my dear friends Vitor and Amanda,

$$
\text { and my cat - 갈비 }
$$




\section{Acknowledgments}

I would like to thank many people who impacted and influenced my stay as a Ph.D. student at West Virginia University - WVU.

First of all, I would like to thank and gratefully acknowledge Dr. Fernando V. Lima, who was a mentor even before he became my Ph.D advisor. His enthusiasm and knowledge in process systems engineering field has led me to where I am now. His guidance and (great) patience made me believe in myself, grow, and make my voice heard. $\mathrm{He}$ motivated me to pursue extra-curricular activities which later enabled me to establish the WVU Graduate Womxn in Chemical and Biomedical Engineering (GWiCBE) student organization. He also taught me not to take everything so seriously, for my heart's sake. I would also like to thank my PhD committee members: Dr. Debangsu Bhattacharyya, whose open-door policy I fully took advantage of; Dr. Richard Turton, for the support and guidance in our meetings; Dr. Stephen Zitney, for the insightful and encouraging ideas; Dr. Jignesh Solanki, for the organizational tips and for motivating me to always look beyond with academic curiosity; and Dr. Urmila Diwekar, whose virtual open-door policy I also fully took advantage in the last few weeks of my Ph.D.

I would like to thank U.S.-China Clean Energy Research Center and U.S. Department of Energy for their funding support throughout my Ph.D. research work. I would like to acknowledge Dr. Lima, Dr. Turton and Dr. Bhattacharyya once again and also Dr. Xin He, Dr. Yifan Wang, Dr. Sai Vudata Pushpitha and (soon to be Dr.) Vitor Gazzaneo for the useful discussion in the project meetings, paper collaborations, and insightful comments.

During my time in the Control, Optimization, and Design for Energy and Sustainability (CODES) research group, I was blessed to interact and see so many Ph.D students become doctors and many undergraduates become graduate students. I sincerely thank all current and past members of the CODES group, Vitor G., Dr. Juan Carlos Carrasco, Dr. Xin He, Dr. Shuyun Li, Dr. Gaurav Mirlekar, Brent, Ron, San, Selorme, Victor A., Thiago, Daniel, Dr. Bispo, Hunter, lan, and Kevin. Their friendship in G-55 and ZOOM meetings kept me sane and helped me finish my Ph.D during a global pandemic. Thanks also to my friends Vitor G., Amanda, Thais, Jordan, Caio, Tadeu, Park, Natalie, Ana Paula, Minji, and many others for making my life during Ph.D so fun. I would also like to thank 
my partner Joshua, whose unwavering trust and support uplifted me so many times and my therapist Dr. Stephanie H., who navigated and helped maintain my mental health throughout the chaotic pandemic.

Finally and most importantly, I would also like to thank my family for supporting me with love all these years. My mother, Kyung An Kwon, whose strength and perseverance I look up to. My father, Dong Ho Kim, who always trusted and believed in me. My sisters, Esther Kim and Hanna Kim, who support me independently of time and place. At last, my 14-year-old cat 갈비, who has been my emotional companion since my last year at highschool in Brazil.

My gratitude to them is immensurable. 


\section{Table of Contents}

Abstract

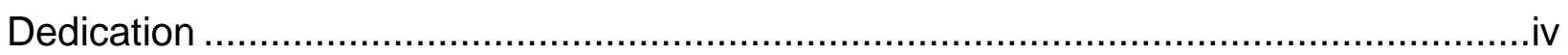

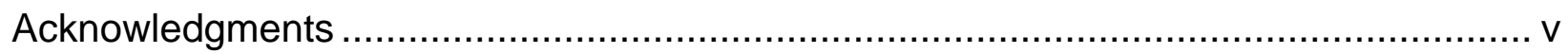

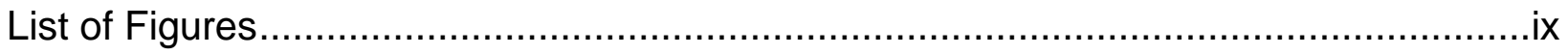

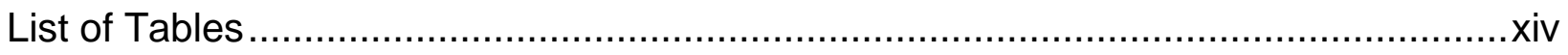

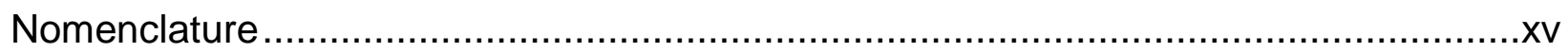

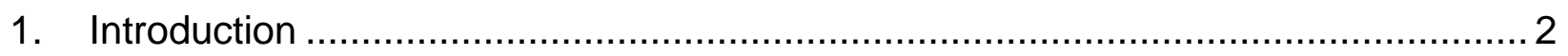

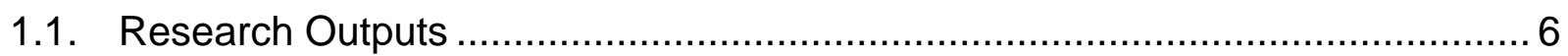

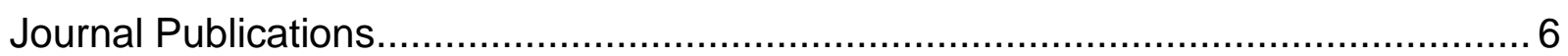

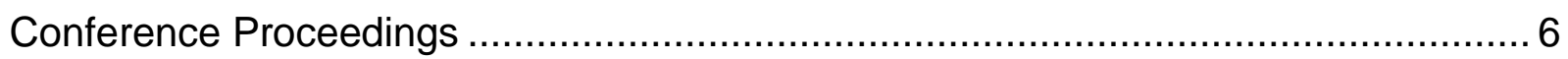

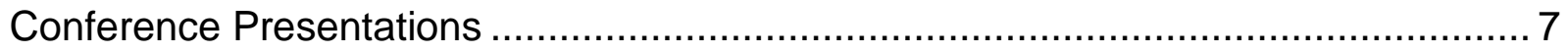

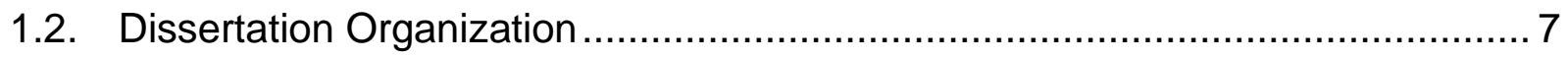

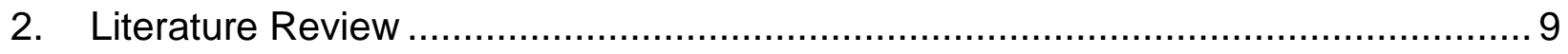

2.1. Hierarchy of Grid Operation and Timeframes............................................. 9

2.2. Particle Swarm Optimization and Parallelized Particle Swarm Optimization in

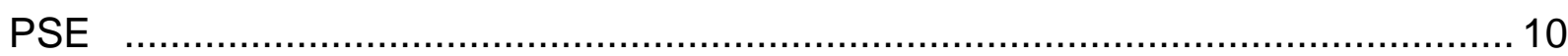

2.3. Multi-objective Optimization in PSE ..................................................... 11

2.4. Dynamic Real-time Optimization in PSE ……......................................... 13

2.4.1. Intersection of DRTO: Brief Review and Terminology Proposition ............ 14

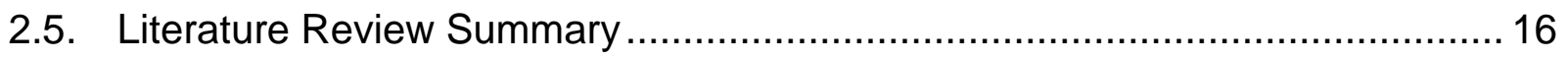

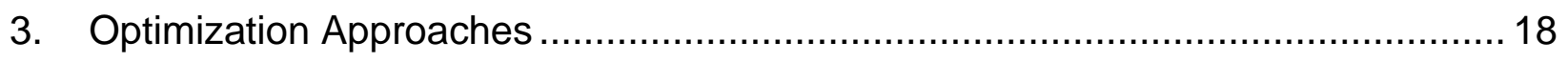

3.1. Nonlinear Multi-objective and Dynamic Real-time Predictive Optimization

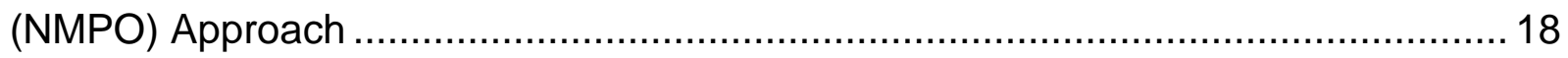

3.1.1. Parallelized Particle Swarm Optimization Formulation ............................ 18

3.1.2. Interior Point Optimizer (IPOPT) Overview .......................................... 22 
3.1.3. Multi-objective Optimization Formulation .............................................. 23

3.1.4. Dynamic Real-time Optimization Formulation....................................... 30

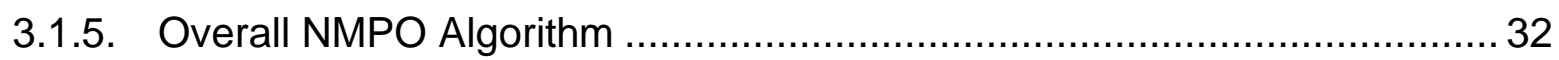

3.2. MILP-based Approach for Dynamic Dispatch of Energy Systems Considering

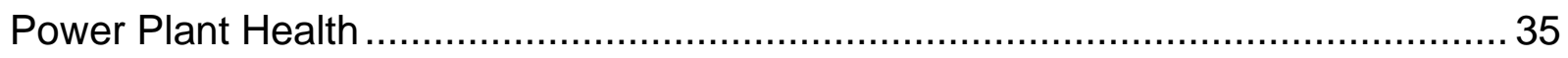

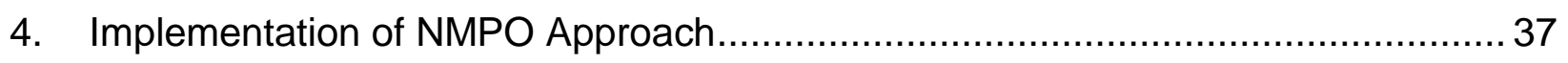

4.1. Supercritical Pulverized Coal-fired Power Plant with MEA-based Carbon

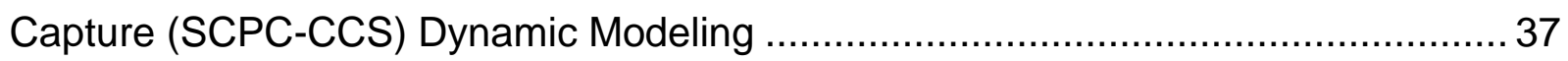

4.2. Nonlinear Optimization of the SCPC-CCS …............................................. 46

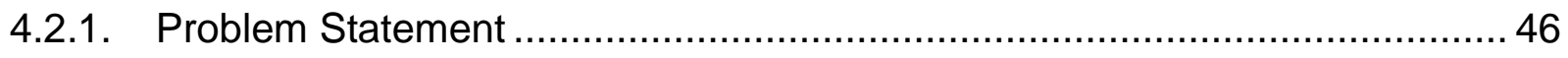

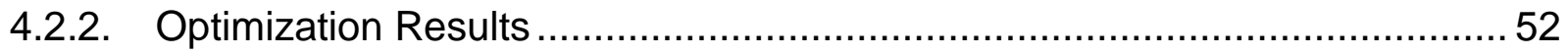

5. Implementation of the MILP-based Dynamic Dispatch considering Power Plant

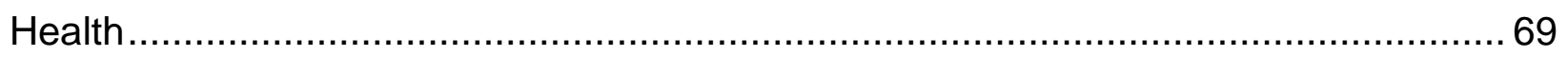

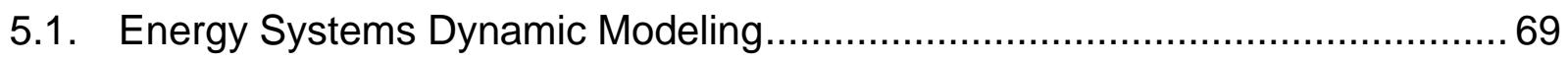

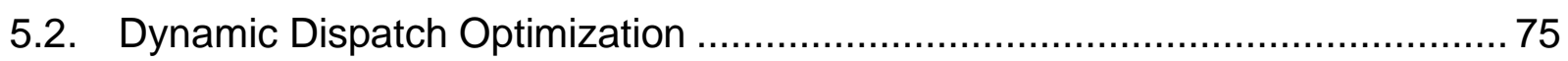

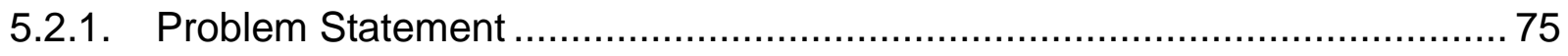

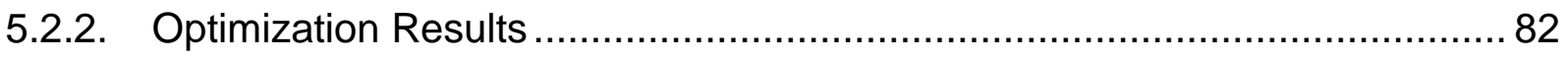

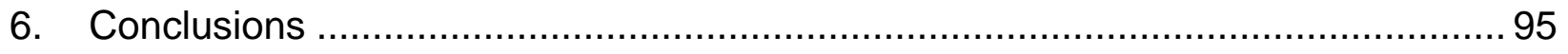

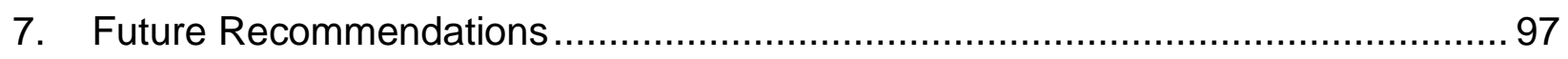

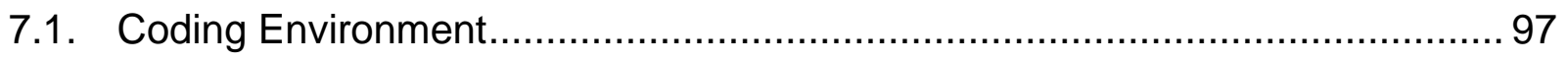

7.2. Optimization Formulation and Connection with MPC-based Controller............99

7.3. Unit Commitment Problem with Equipment Health-related Constraints and

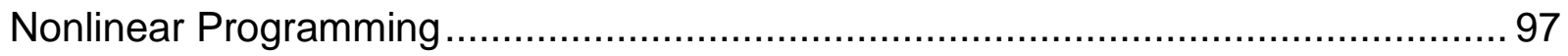

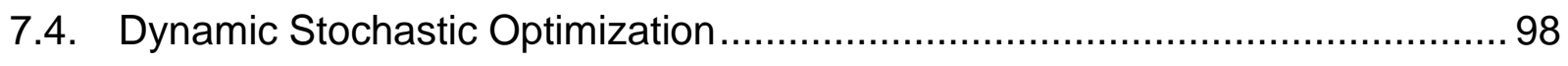

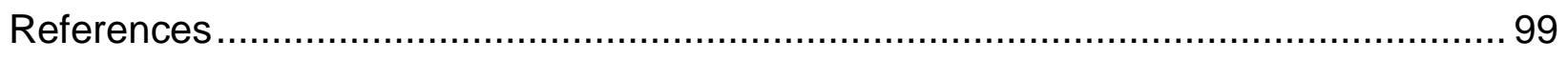




\section{List of Figures}

Figure 1.1. Power load cycling profile for the Cherokee power plant during a wind event on July $2^{\text {nd }}, 2008$ (adapted from Bentek, 2010)

Figure 1.2. Schematic of the interconnected optimization framework considering power plants, intermittent renewables, power economics, and demand....

Figure 2.1. Illustration of the hierarchical multiscale grid operations (adapted from US DOE, 2017) 10

Figure 2.2. a) RTO paired with MPC, b) RTO paired with distributed MPC, c) DRTO paired with MPC, d) DRTO paired with distributed MPC, e) EMPC optionally pairing with RTO, f) DRTO with MPC subproblems paired with distributed MPC ..................................... 16

Figure 3.1. Schematic of the PSO update mechanism in 2-dimensional space ............ 20 Figure 3.2. Pseudocode of synchronous and asynchronous ParPSO approaches ....... 22 Figure 3.3. Weighted metric method search mechanism for different $p$ values (adapted

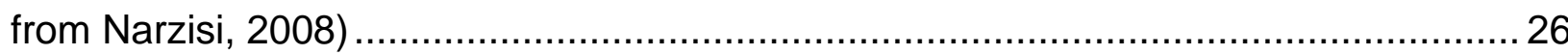

Figure 3.4. $\epsilon$-constrained method search mechanism (adapted from Narzisi, 2008)..... 29

Figure 3.5. Schematic of proposed optimization framework implementation................. 33

Figure 3.6. Pseudocode for the implementation of the NMPO framework..................... 35 Figure 4.1. Supercritical coal-fired power plant with the MEA-based carbon capture ... 39 Figure 4.2. Comparison between 1-hour ahead model prediction with data from highfidelity Aspen model - CCS nonlinear ARX. 44

Figure 4.3. Comparison between 1-hour ahead model prediction with data from highfidelity Aspen model - SCPC linear ARX 45

Figure 4.4. Comparison between 1-hour ahead model prediction with data from highfidelity Aspen model - SCPC nonlinear ARX 46

Figure 4.5. Power demand cycling profile (left axis), electricity price and carbon credit (right axis) for 12-hour horizon .... 49

Figure 4.6. Objective values for implementations with different optimization horizons .. 51 Figure 4.7. Computational times for implementations with different optimization horizons (dots) and the considered real-time constraint (line) ..... 51 Figure 4.8. Feasible space and distribution of optimal compromise and the optimal compromise according to the weight vector along the Pareto front 53 
Figure 4.9. Satisfied SCPC power output constraint

Figure 4.10. Economic objective value for optimization horizon of the economic, environmental, and MTch multi-objective optimizations - Case 1 55 Figure 4.11. Economic objective value for implemented horizon of the economic, environmental, and MTch multi-objective optimizations - Case 1 .............................. 55 Figure 4.12. Environmental objective value for optimization horizon of the economic, environmental, and MTch multi-objective optimizations - Case 1 ...............................56 Figure 4.13. Environmental objective value for implemented horizon of the economic, environmental, and MTch multi-objective optimizations - Case 1 ...............................56 Figure 4.14. Economic objective value for optimization horizon of the economic, environmental, and MTch multi-objective optimizations - Case 2 ..............................56 Figure 4.15. Economic objective value for implemented horizon of the economic, environmental, and MTch multi-objective optimizations - Case 2 ..............................5 56 Figure 4.16. Environmental objective value for optimization horizon of the economic, environmental, and MTch multi-objective optimizations - Case 2 …..........................5 57 Figure 4.17. Environmental objective value for implemented horizon of the economic, environmental, and MTch multi-objective optimizations - Case 2 .............................5 57 Figure 4.18. Main steam operating pressure for economic, environmental and MTch multiobjective optimizations - Case 1. 58

Figure 4.19. Main steam operating temperature for economic, environmental and MTch multi-objective optimizations - Case 1 ...... 58 Figure 4.20. Main steam operating pressure for economic, environmental and MTch multiobjective optimizations - Case 2 59 Figure 4.21. Main steam operating temperature for economic, environmental and MTch multi-objective optimizations - Case 2 59 Figure 4.22. Coal feedrate for Cases 1 and 2 obtained with MTch multi-objective optimization 59 Figure 4.23. CND pump power for Cases 1 and 2 obtained with MTch multi-objective optimization...... 60 Figure 4.24. BFW pump power for Cases 1 and 2 obtained with MTch multi-objective optimization 60 
Figure 4.25. Low-pressure steam flowrate for Cases 1 and 2 obtained with MTch multiobjective optimization

61

Figure 4.26. Lean solvent flowrate for Cases 1 and 2 obtained with MTch multi-objective optimization 61

Figure 4.27. Main steam flowrate for Cases 1 and 2 obtained with MTch multi-objective optimization

Figure 4.28. CCS equivalent work for Cases 1 and 2 obtained with MTch multi-objective optimization .... 62

Figure 4.29. Carbon captured for Cases 1 and 2 obtained with MTch multi-objective optimization 63

Figure 4.30. Lean solvent $\mathrm{CO}_{2}$ loading for Cases 1 and 2 obtained with MTch multiobjective optimization 63

Figure 4.31. Illustrative example of the "bang-bang" effect in the NMPO framework .... 65 Figure 4.32. Economic objective value for optimization horizon of the economic, environmental, and $\epsilon$-constraint multi-objective optimizations - Case 1 66 Figure 4.33. Economic objective value for implemented horizon of the economic, environmental, and $\epsilon$-constraint multi-objective optimizations - Case 1. 66 Figure 4.34. Environmental objective value for optimization horizon of the economic, environmental, and $\epsilon$-constraint multi-objective optimizations - Case 1 66 Figure 4.35. Environmental objective value for implemented horizon of the economic, environmental, and $\epsilon$-constraint multi-objective optimizations - Case 1 66 Figure 4.36. Economic objective value for optimization horizon of the economic, environmental, and $\epsilon$-constraint multi-objective optimizations - Case 2 .... 67 Figure 4.37. Economic objective value for implemented horizon of the economic, environmental, and $\epsilon$-constraint multi-objective optimizations - Case 2 ..... 67 Figure 4.38. Environmental objective value for optimization horizon of the economic, environmental, and $\epsilon$-constraint multi-objective optimizations - Case 2 . 67

Figure 4.39. Environmental objective value for implemented horizon of the economic, environmental, and $\epsilon$-constraint multi-objective optimizations - Case 2 ......................6 67 Figure 5.1. Schematic of the SCPC power plant (adapted from Kim et al., 2020) .........69 69 Figure 5.2. Schematic of a single NaS cell (adapted from Kim et al., 2020)..... 69 
Figure 5.3. Schematic of the NGCC power plant (adapted from Kim et al., 2020) ........ 70

Figure 5.4. Representation of the wind and solar (adapted from Kim et al., 2020)........ 70

Figure 5.5. Plot of capacity (Wh) vs. state of discharge of a single NaS cell ................. 70

Figure 5.6. Plot of state of discharge vs. time for a single NaS cell............................. 71

Figure 5.7. Comparison between 6 minute ahead model prediction with data from highfidelity Aspen model - SCPC total power output ....................................................... 73

Figure 5.8. Comparison between simulations and data from high-fidelity Aspen model -

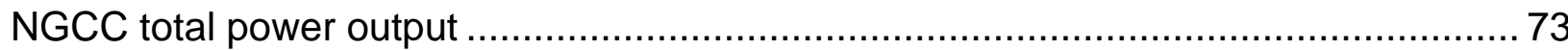

Figure 5.9. Comparison between simulations and data from high-fidelity Aspen model -

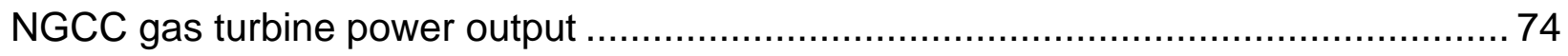

Figure 5.10. Comparison between simulations and data from high-fidelity Aspen model NGCC main steam temperature 74

Figure 5.11. Comparison between simulations and data from high-fidelity Aspen model NGCC gas reheat steam temperature. 74

Figure 5.12. Comparison between simulations and data from high-fidelity Aspen model NGCC drum mechanical stress 74

Figure 5.13. Comparison between simulations and data from high-fidelity Aspen model NGCC drum thermal stress 75

Figure 5.14. 2019 total and modified electricity demand load ................................ 77

Figure 5.15. 2019 and 2050 variable renewable energy penetrations (solar/wind) ....... 77

Figure 5.16. Diagram of a steam drum (including the drum-downcomer junction) ........ 80

Figure 5.17. Power dispatch optimization result for Case 1 ...................................... 83

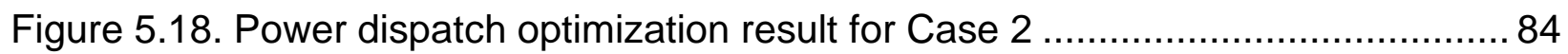

Figure 5.19. Power dispatch optimization result for Case 3 .................................... 85

Figure 5.20. 2050 renewable levels accepted and curtailed for Case 3 ....................... 85

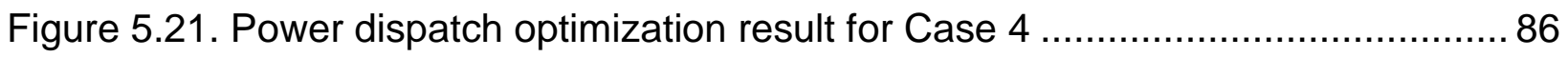

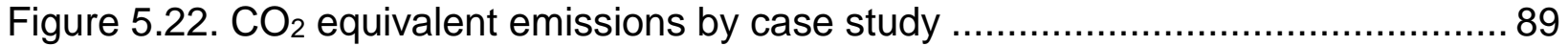

Figure 5.23. Drum body and concentrated tangential stresses during dispatch for Cases

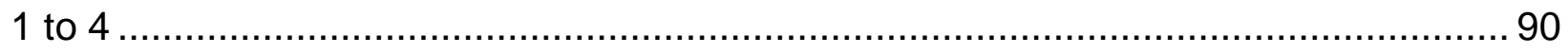

Figure 5.24. Modified electricity demand load for Case 5 ....................................... 91

Figure 5.25. Modified variable renewable energy penetrations (solar/wind) for Case 591 
Figure 5.26. Sensitivity analysis of optimization objective due to NGCC and NGCC 2

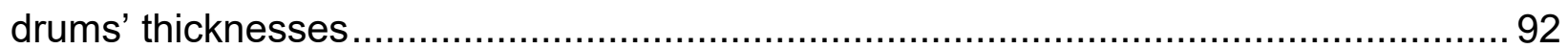

Figure 5.27. Power dispatch optimization result for Case 5 .................................... 93

Figure 5.28. Drum body and concentrated tangential stresses during dispatch for Case 5 93

Figure 5.29. Mechanical and thermal stresses for NGCC for Case 5 .......................... 94

Figure 5.30. Mechanical and thermal stresses for NGCC 2 for Case 5....................... 94 


\section{List of Tables}

Table 4.1. Decision and dependent variables for the SCPC-CCS system

Table 4.2. Regressors of the linear and nonlinear ARX models for SCPC-CCS and correspondence between data and 1-hour ahead predictions.................................... 43

Table 4.3. ParPSO parameters for the NMPO framework........................................ 49

Table 4.4. Optimization parameters for carbon policy scenarios - Cases 1 and 2 2....... 54

Table 5.1. Electricity generation from selected fuels (EIA, 2019a) ............................. 76

Table 5.2. High-pressure drum specification with $\mathrm{MOC}^{1}$ being SA-515 grade $70 \ldots \ldots . . .78$

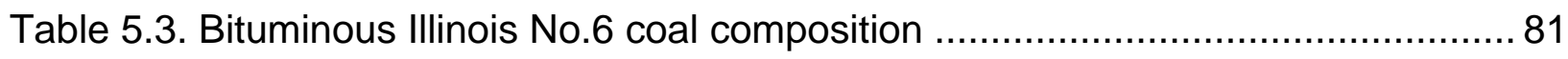

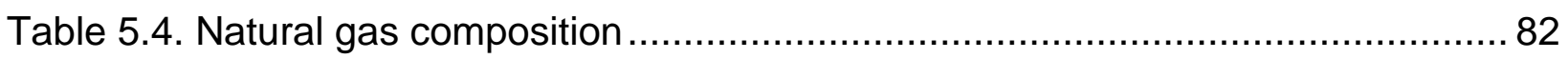




\section{Nomenclature}

\section{Notation}

$a_{1}, \ldots, a_{n_{a}}, b_{1}, \ldots, b_{n_{b}}-$ regression coefficients

$A_{N G C C}, B_{N G C C}, C_{N G C C}, D_{N G C C}-\mathrm{NGCC}$ system matrices

as - scaling coefficient vector

aw - wavelet coefficient vector

bs - scaling dilation vector

bw - wavelet dilation vector

$c$-general nonlinear constraints

$c_{1}-$ cognitive acceleration coefficient

$c_{2}$ - social acceleration coefficient

Carbon credit - value for the carbon captured in a potential cap\&trade scenario

Carbon Captured - amount of $\mathrm{CO}_{2}$ captured

Coal price - price of the coal

Carbon tax - embedded tax in the carbon released into the atmosphere

CCmarket - hypothetical change in the market price value of the $\mathrm{CO}_{2}$ credit $h_{4, \text { TAX }}-\operatorname{tax}$ value imposed to the amount of carbon released

cs - scaling translation matrix

$c W-$ wavelet translation matrix

$d$ - output offset scalar

$d_{m b}-$ branch mean diameter

$d_{m s}$ - main body mean diameter

$E$ - Joint efficiency of the shell-branch connection 
Electricity - price of the electricity in wholesale

$E O R$ - credit due to $\mathrm{CO}_{2}$ utilization for enhanced oil recovery

$f$ - system dynamic model

$f c-N A R X$ nonlinear mapping

$h$ - equality constraints

$h_{2}$ - cost for onshore $\mathrm{CO}_{2}$ transportation pipelines

$h_{3}$ - cost for onshore $\mathrm{CO}_{2}$ storage

$h_{5}-$ value for enhanced oil recovery

$h_{s}-$ equality constraints after adding slack variables

$h_{t}-$ Thermal transmittance for steam

$g-$ inequality constraints

$I T$ - inertia term

$L$ - linear coefficient

maxiter - maximum number of PSO iterations

$n_{a}-$ number of poles

$n_{b}$ - number of zeros

$n_{k}$ - deadtime of the system

$n_{s}, n_{w}-$ number of scaling and wavelet units

$n_{x}, n_{y}-$ maximum input and output lags

$p$ - parameter between $[1, \infty]$

$p_{j}-$ position of the $\mathrm{j}$-th particle of the swarm

$P B_{j}$ - personal best position of the $\mathrm{j}$-th particle of the swarm

$Q, P$ - nonlinear and linear projection matrices 
$r$ - mean of $\chi$ vector

$r_{1}, r_{2}-$ random numbers between $[0,1]$.

$r_{\text {inb }}-$ Branch radius

$r_{\text {inms }}$ - Main body radius

$s$ - slack variables

$s_{b}-$ Branch thickness

$s_{m s}$ - Main body thickness

SB - swarm best position

Storage - cost related to $\mathrm{CO}_{2}$ storage

$T$ - time horizon

$t$ - time or timestep

$t_{o}-$ initial time

$t_{1}$ - implementation time horizon

$t_{2}$ - optimization time horizon

Transportation - cost related to $\mathrm{CO}_{2}$ transportation

$u^{*}$ - ideal vector

$u_{N G C C}-$ NGCC input vector

$v_{j}-$ velocity of the j-th particle of the swarm

$w$ - weight vector

$w_{\mathrm{i}}^{\mathrm{e}-\text { const }}-$ weight vector for the e-constraint method

wn - white noise.

$X-$ decision space

$x$ - decision/input variables 
$x_{1}-$ Coal feedrate

$x_{2}-$ Air flowrate

$x_{3}-$ CND pump power

$x_{4}-$ Main steam pressure

$x_{5}-$ BFW pump power

$x_{6}-$ Low-pressure steam flowrate

$x_{7}-$ Lean solvent flowrate

$x_{N G C C}-$ NGCC state vector

$x_{s}-$ Decision variables augmented with slack variables

$y$ - Dependent/output variables

$y_{1}$ - Power output

$y_{2}-$ Main steam temperature

$y_{3}-$ Flue gas flowrate

$y_{4}-\mathrm{CO}_{2}$ in flue gas flowrate

$y_{5}$ - efficiency of low-pressure turbine $1-\eta\llcorner P 1$

$y_{6}$ - efficiency of low-pressure turbine 2 - nLP2

$y_{7}$ - Main steam flowrate

$y_{8}-\mathrm{CO}_{2}$ capture rate

$y_{9}-$ Lean solvent $\mathrm{CO}_{2}$ loading

$y_{10}-$ CCS overall work

$y_{\text {data }}$ - Dependent/output data

$\hat{y}_{\text {prediction }}$ - Dependent/output prediction by the reduced-order model 
$\bar{y}_{\text {data }}$ - Dependent/output data average

$y_{N G C C}-$ NGCC output vector

$Z$ - Objective space

$z$ - Objective vector

\section{Acronyms}

$A D$ - Automatic Differentiation

ADiMAT - Automatic Differentiation for MATLAB

APD - Aspen Plus Dynamics

ARX - Autoregressive with Exogenous Inputs

BFGS - Broyden-Fletcher-Goldfarb-Shanno

BFW - Boiler Feedwater

CCS - Carbon Capture System

CND - Condenser

DCC - Direct Contact Cooler

D-RM - Dynamic Reduced Models

DRTO - Dynamic Real-Time Optimization

ECON - Economic

EMPC - Economic Model-based Predictive Control

ES - Dispatchable Energy Set

ENV - Environmental

FOM - Full-order model

GOF - Goodness of Fit

IPOPT - Interior Point Optimizer 
LB - Lower bound

MAS - Maximum Allowable Stress

MILP - Mixed-integer Linear Programming

MOC - Material of construction

MOO - Multi-Objective Optimization

MPC - Model Predictive Control

NARX - Nonlinear Autoregressive with Exogenous Inputs

NERC - North American Electric Reliability Corporation

NGCC - Natural-gas Combined Cycle Power Plant

NMPO - Nonlinear Multi-objective and dynamic real-time Predictive Optimization

NRMSE - Normalized Root Mean Squared Error

MTch - Modified Tchebycheff weighted metric

ParPSO - Parallelized Particle Swarm Optimization

PSE - Process Systems Engineering

PSO - Particle Swarm Optimization

ROM - Reduced-order model

SCPC - Supercritical Pulverized Coal-fired Power Plant

SOD - State of Discharge

SQP - Sequential Quadratic Programming

Tch - Tchebycheff weighted metric

UB - Upper bound

UC - Unit Commitment

VRE - Variable Renewable Energy 


\section{Greek letters}

$\alpha$ - extra variable for minmax formulation

$\varepsilon$ - independent identically distributed random variable

$\varepsilon_{1}-$ NaS discharge model deviation term

$\epsilon_{i}$ - parametric value of the $\mathrm{i}$-th objective that it is not the k-th objective

$\eta_{L P_{1}, L P_{2}}$ - efficiencies of low-pressure turbine $(1,2)$

$\theta-$ scaling function

$\lambda_{m}-$ mechanical stress concentration factor

$\lambda_{t}-$ thermal stress concentration factor

$v$ - integer variables

$\sigma_{\theta, b o d y}$ - overall tangential stress

$\sigma_{\theta, b o d y}^{P}-$ tangential stress due to pressure (mechanical stress)

$\sigma_{\theta, b o d y}^{T}$ - tangential stress due to temperature (thermal stress)

$\Phi_{\mathrm{n}}-$ objective function(s)

$\chi$ - vector of regressors

$\psi$ - wavelet function

$\Omega, \Omega_{1}, \Omega_{2}, \gamma$ - constriction factors 


\section{Dynamic Optimization Algorithms for Baseload Power Plant Cycling under Variable Renewable Energy}




\section{Introduction}

Changes in global climate, societal and cultural pressures, in conjunction with the decrease of renewable technologies cost, stimulated an increase of variable renewable energy (VRE) participation in the power generation grid (REN21, 2018). VRE is defined here as any type of renewable energy that cannot be dispatched as needed, i.e., the energy is only available when the fuel source is naturally available, and usually presents intermittency in power output (EIA, 2019a). The most common VRE sources are solar and wind, which are becoming more cost competitive with solar technology costs falling 85\% and wind costs falling 36\% between 2008 and 2016 (Houser et al., 2017). Besides being non-dispatchable technologies when not coupled with energy storage, solar and wind are also known to have limited alignment with electricity demand (USAID-NREL Partnership, 2019). In other words, the period that the VRE is available does not necessarily coincides when the electricity demand peaks. Therefore, to maintain the reliability of energy supply, dispatchable technologies need to be used to supply the net load.

Due to the introduction of such disruptive VRE technologies, even fossil-fired power plants originally designed to operate in constant power output, i.e., baseload thermal power plants, need to cycle their load to maintain the power balance (Bentek, 2010). The cycling of baseload power plant is not ideal, as power plants must be ramped up/down to accommodate the variability. Baseload capacity is mostly provided by coal-fired and nuclear power plants, with the more modern natural gas power plants often performing as load-following power plants. Between nuclear and coal technologies, the baseload coal-fired power plants are chosen to cycle their load due to safety reasons. For instance, Figure 1.1 shows the load profiles from the gas- and coal-fired Cherokee Power Station, in which the coal power generation needs to significantly and rapidly ramp up and down to accommodate the wind event (circled portions), while the gas power generation provides the peak load.

Some of the main concerns associated with an increase of unexpected power plant cycling are: 1) the required power plant minimum operating loads; 2) environmental performance during cycling due to loss of governance of the pollution control units; and 
3) prohibitive ramping rates and lower efficiency, which may increase unplanned operations and maintenance costs (Bentek, 2010; Grol et al., 2015; Wang et al., 2020).

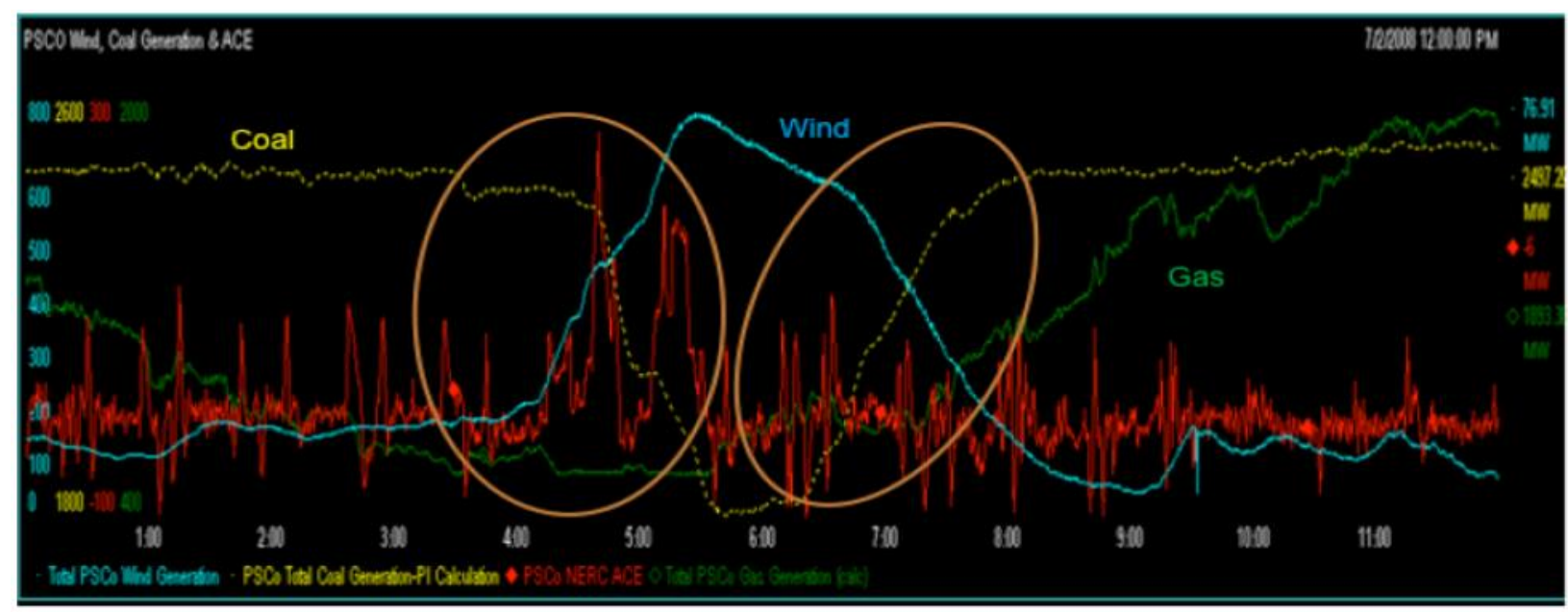

4:00 AM

8:00 AM

Figure 1.1. Power load cycling profile for the Cherokee power plant during a wind event on July $2^{\text {nd }}, 2008$ (adapted from Bentek, 2010)

Considering the challenging task of operating and controlling cycling power plants, the optimal operation of these power plants has recently been a topic of research in the Process Systems Engineering field (Dowell and Shah, 2014; Bankole et al., 2018; He and Lima, 2020). The optimization approach should be implemented at different time scales from the power system operations. In the unit commitment level, operation planners schedule enough units to meet the forecasted demand and manage the reserves, usually between six hours to a few days ahead. Throughout the day, operators and traders usually give hourly schedules to units and will make trades based on economics. As real-time approaches, operators adjust generator schedules to dispatch the quickly changing demand. Units with sufficient ramping capabilities are often used to meet demand on a five-minute to one-hour time basis (Ela and Kemper, 2009).

Therefore, considering that the power plant never reaches steady state due to cycling, the dynamics of these energy systems become relevant to determine the optimal operation policies. Additionally, more than one stakeholder should be considered as the economic, environmental, and power plant health performances are affected during cycling. The North American Electric Reliability Corporation (NERC) has reported that the 
three main identified roots of disturbances in the grid are management/organization (40\%), design/engineering (30\%), and equipment/material (11\%) (NERC, 2020). These three main drivers can at some level be addressed using process systems engineering strategies.

A general optimization and control framework is presented in Figure 1.2. In this framework, the optimizer can play a role at each time scale (daily, hourly, and minute) considering the forcing function information such as forecasted demand, renewable penetration, and power economics, as well as power plant current state and health.

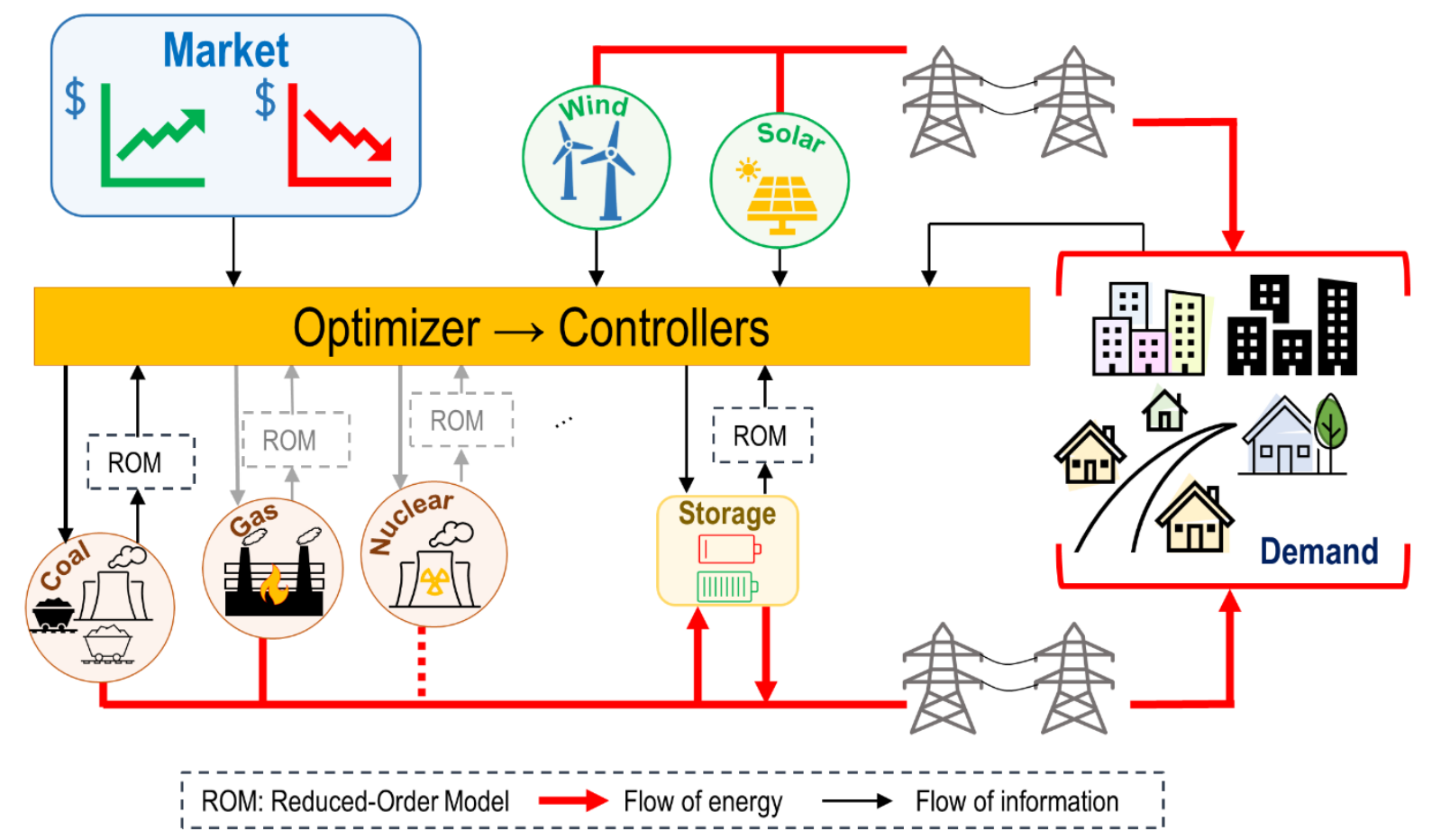

Figure 1.2. Schematic of the interconnected optimization framework considering power plants, intermittent renewables, power economics, and demand

In this context, dynamic optimization that determines optimal power plant output trajectories considering the system dynamics and constraints while minimizing overall objectives is desirable. The performed dynamic optimization can be both linear and nonlinear, as long as the solution can be obtained in real time. The shorter is the time scale, the faster the problem needs to be solved. 
The objective in this research is to design and implement dynamic optimization algorithms for baseload power plant cycling under variable renewable energy penetration. Based on the power generation time scale, dynamic optimization frameworks are developed for the hourly and minute-basis time scales. The following specific aims are addressed in this research:

1) Develop a Dynamic Real-Time Optimization (DRTO) approach for systems subject to variable conditions such as VRE penetration, electricity market prices, and electricity demand. This approach involves the development of dynamic reduced-order models and calculation of optimal output trajectories for online implementation on selected baseload energy systems, such as a carbon capture system (CCS) and supercritical pulverized coal-fired (SCPC) power plants. It also includes the development of a parallelized particle swarm optimization (ParPSO) method in MATLAB environment to initialize the algorithm while learning from the feasible space. A theoretical contribution on the intersection of DRTO with MPC and terminology is also proposed.

2) Augment the DRTO with a Multi-Objective Optimization (MOO) method for systems subjected to the same variable conditions as the DRTO but also taking into account different objectives, e.g., economic and environmental, to find the optima. This approach involves the development of strategies to determine Pareto-optimal trajectories that can be implemented with minimal interaction with the decision maker for online application purposes to selected baseload energy systems, such as a carbon capture system (CCS) and supercritical pulverized coal-fired (SCPC) power plants.

3) Design a mixed-integer and disjunctive programming dynamic optimization approach for dispatch of a suite of energy systems under VRE, such as storage units, natural gas-fired and coal-fired power plants. This approach incorporates power plant health-related constraints during dispatch.

4) Establish an optimization strategy for power plants during cycling. Optimal operation guidance is provided to the power plant clients based on the developed optimization approaches. 


\subsection{Research Outputs}

The specific contributions of this dissertation are: i) development of nonlinear DRTO with economic and environmental objectives for a cycling SCPC plant; ii) develop a Nonlinear Multi-objective dynamic-real time Predictive Optimization (NMPO) framework that determines Pareto-optimal trajectories with minimal interaction with decision maker for large-scale baseload energy systems during cycling; iii) inclusion of power plant health constraint during energy system dispatch under VRE; and iv) theoretical discussion on the intersection of DRTO with MPC in the process systems engineering (PSE) field.

The contributions of this dissertation have resulted in the following products: $\underline{\text { Journal Publications }}$

1. Rebecca Kim and Fernando V. Lima. Nonlinear Multi-objective and dynamic real-time Predictive Optimization (NMPO) for Optimal Operation of Baseload Power Plants under Variable Renewable Energy. In preparation for publication.

2. Rebecca Kim, Yifan Wang, Sai Pushpitha Vudata, Debangsu Bhattacharyya, Fernando V. Lima, and Richard Turton, 2020. Dynamic Optimal Dispatch of Energy Systems with Intermittent Renewables and Damage Model. Mathematics 8(868). doi:10.3390/math8060868.

3. Rebecca Kim and Fernando V. Lima, 2020. A Tchebycheff-based multi-objective combined with a PSO-SQP dynamic real-time optimization framework for cycling energy systems. Chemical Engineering Research and Design 156:180-194, doi: 10.1016/j.cherd.2020.01.020.

\section{Conference Proceedings}

1. Sisi Guo, Rebecca Kim, Fernando V. Lima, Pei Liu, Zheng Li., 2017. Dynamic data reconciliation and optimization methods in coal-fired power plants. In Proceedings of the 2017 International Conference on Coal Science \& Technology and 2017 AustraliaChina Symposium on Energy, September 2017. 


\section{$\underline{\text { Conference Presentations }}$}

1. Rebecca Kim, Sai Pushpitha Vudata, Yifan Wang, Debangsu Bhattacharyya, Fernando V. Lima and Richard Turton. Optimal Dispatch of Energy Systems Considering Penetration of Renewables and Power Plant Health. Oral Presentation at AIChE Annual Meeting, Virtual, November 2020.

2. Rebecca Kim and Fernando V. Lima. Large-scale Dynamic and Multi-Objective Optimization for Power Plant Cycling. Oral Presentation at AIChE Annual Meeting, Virtual, November 2020.

3. Rebecca Kim, Sai Pushpitha Vudata, Yifan Wang, Debangsu Bhattacharyya, Fernando V. Lima and Richard Turton. Scheduling of Baseload Power Plants and Batteries with Integration of Renewables. Poster Presentation at AIChE Annual Meeting, Orlando, FL, USA, November 2019.

4. Rebecca Kim and Fernando V. Lima. Multi-objective and Dynamic Real-time Optimization of Supercritical Coal-fired Power Plant Cycling. Oral Presentation at AIChE Annual Meeting, Orlando, FL, USA, November 2019.

5. Ronald Alexander, Rebecca Kim, and Fernando V. Lima. Simulation, Economic Optimization, and Control of an Autorefrigerated Alkylation Process. Poster Presentation at AIChE Annual Meeting, Pittsburgh, PA, USA, November 2018.

6. Rebecca Kim and Fernando V. Lima. Multi-objective and Dynamic Real-time Optimization for Postcombustion Carbon Capture Processes for Cycling Application. Oral Presentation at AIChE Annual Meeting, Pittsburgh, PA, USA, November 2018.

7. Rebecca Kim and Fernando V. Lima. Nonlinear System Identification and Dynamic Real Time Optimization of Postcombustion $\mathrm{CO}_{2}$ Capture Processes for Cycling Applications. Poster Presentation at AIChE Annual Meeting, Minneapolis, MN, USA, November 2017.

\subsection{Dissertation Organization}

The outline for the remaining chapters of this dissertation includes a general literature review, which is presented in Chapter 2. The theoretical intersection of DRTO with MPC in PSE field is also presented in Chapter 2. All the optimization approaches, which includes the DRTO, the modified Tchebycheff MOO, the $\epsilon$-constraint MOO, the ParPSO, 
and the mixed-integer linear programming (MILP)-based dynamic dispatch, are summarized in Chapter 3. In Chapter 4, two NMPO formulations are implemented in the SCPC with aqueous monoethanolamine (MEA)-based CCS. In Chapter 5, the MILPbased dynamic dispatch algorithm is implemented for a suite of energy systems under various VRE scenarios. This suite of energy systems includes the natural-gas combined cycle (NGCC) power plant, the SCPC, sodium sulfur batteries, and wind and solar input as renewable sources. Finally, the overall conclusions of the dissertation are outlined in Chapter 6 and recommendations for future work are addressed in Chapter 7. 


\section{Literature Review}

This chapter presents a brief overview of the literature reviewed prior and during this research, following the main topics: 1) hierarchy of grid operation timeframes; 2) particle swarm optimization and parallelized particle swarm optimization in PSE; 3) multi-objective optimization in PSE; and 4) dynamic real-time optimization in the PSE field.

\subsection{Hierarchy of Grid Operation and Timeframes}

The time scale of the decision-making process in grid operation ranges from milliseconds to decades, a $10^{12}$ s order range (US DOE, 2017, Pattison et al., 2016). Figure 2.1 shows the main components of the hierarchical multiscale events that occur in grid operations. Overall, the timeframe can be divided in three sections:

i) long-term planning: ranges from decade to months. The main focus is on expanding the grid capacity and transmission lines, planning of power plant construction and decommissioning, and set the necessary conditions to achieve long-term goals such as the carbon and grid resilience goals to withstand challenging conditions.

ii) mid-term planning: ranges from weeks to minutes. The focus here is on managing the installed energy portfolio, scheduling and dispatching existing energy systems, and responding to disruptive penetration of variable renewable energy as well as demand variability to maintain constant power supply. At this level, advanced model-based controllers, real-time and dynamic real-time optimization, and unit commitment problems can be solved to enhance grid performance.

iii) regulatory response: ranges from minutes to seconds. Then, the focus of this time scale is on implementing the setpoints/trajectories planned in the higher order time scale using basic controllers. Protective relay operations also perform in this time scale. At this level, the priority is to safely operate the energy systems, implement the previously calculated planned trajectories, and respond to unexpected events at the millisecond to second range, if needed. 


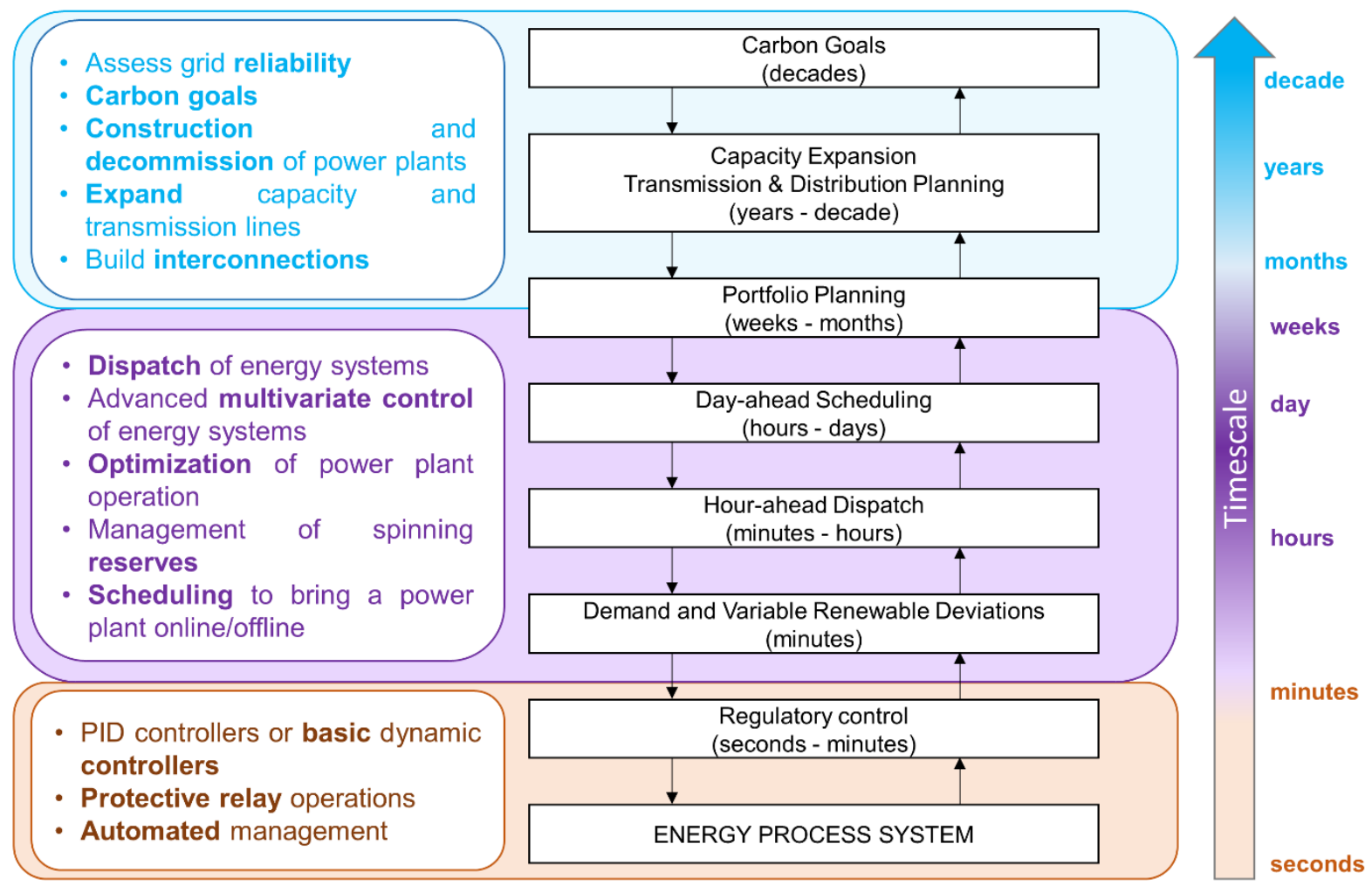

Figure 2.1. Illustration of the hierarchical multiscale grid operations (adapted from US DOE, 2017)

Traditional system operations function within this framework. However, with the recent changes in energy supply, they should adapt to enable a more dynamic and integrated grid by growing interconnections, communications, and data flows, while addressing cybersecurity vulnerabilities, reliability, and resilience.

\subsection{Particle Swarm Optimization and Parallelized Particle Swarm Optimization in} PSE

The mathematical definition of optimization is, given a system or process, to find the best solution of the process in a systematic manner while considering the system constraints. The quality of the solution is assessed by a set of objectives.

A meta-heuristic optimization also known as gradient-free optimization is an algorithm that relies on a heuristic set of rules to generate those solutions. Meta-heuristic programming does not require convexity or derivatives information. The rules are often 
inspired on nature and physical phenomena, such as ant-colony optimization, simulated annealing, genetic and evolutionary algorithms, and particle swarm optimization.

Particularly, the particle swarm optimization is inspired on the social behavior and communication within a bird flocking. The PSO is known to be simple to implement as the update mechanism is essentially guided by two rules and does not involve evolution operators such as crossover and mutation. However, as with any population-based optimization, the PSO relies in a large number of iterations to find the optima which then leads to higher computational times. Due to this limitation, the PSO is largely used to solve offline, steady-state, or low-dimensional size problems, when computational time is not a constraint (Elaiw et al., 2013; Sharafi and EIMekkawy, 2014; Sengupta et al., 2018; Pang et al., 2019).

On the other hand, the simplicity of the PSO facilitates its combination with other optimization techniques. Furthermore, the solution of a problem solved only using a gradient-based optimization algorithm can be sensitive to the initial guess if the problem has multiple local optimal (Elaiw et al., 2013). A multi-start approach could be adopted to address this challenge, which is when the gradient-based algorithm is initiated with different initial guesses and the best solution is retrieved from the pool of solutions. The authors of the cited reference instead propose the hybridization of the PSO with the sequential quadratic programming (SQP) to solve a dynamic economic and environmental dispatch problem with ramping constraints.

In addition to combining the PSO with gradient-based techniques, the PSO algorithm can be easily parallelized in different ways with various topologies to improve the computational time and solve a problem that is time sensitive (Venter and Sobieszczanski-Sobieski, 2005; Sengupta et al., 2018; Pang et al., 2019).

\subsection{Multi-objective Optimization in PSE}

As in many PSE problems, the optimization of cycling energy systems under VRE has more than one stakeholder that should be considered during the decision-making process, such as economic, environmental, safety, and power plant health. In this type of problems, a multi-objective optimization formulation should be employed to return optimal 
compromises between objectives (Diwekar, 2008). However, often only an economic objective is considered during the optimization performed by grid operators.

For instance, during the winter of 2021, the Texas Interconnection managed by the Electric Reliability Council of Texas (ERCOT) faced one of the most challenging weather conditions to date. The conditions were caused by the polar vortex spilling into lower latitudes partly due to the global climate change (Weber and Bleed, 2021). The extreme cold weather affected several power plant equipment and pipelines that were not properly conditioned (ERCOT, 2021a) and ERCOT announced that about $30 \mathrm{GW}$ of generation were forced off the system (ERCOT, 2021b). Therefore, rolling blackouts were implemented to curb demand, lowering the strain on power grids, and left more than an estimated 3 million customers without power (Weber and Bleed, 2021). Previous weather events show that failure to supply energy during extreme weather events have already been suffered by ERCOT in the past, such as the outages in 2011 and 1989 (FERC, 2011). Particularly, the Texas electricity market is known to be unregulated, i.e., customers can pick their electricity providers in a purely market-driven system. However, the analysis of the most recent outage event indicate that the lack of equipment conditioning, which caused the operational inadequacies, can be mostly attributed to cutdown of operational and maintenance costs and lack of preparedness incentives. In other words, the sole minimization of costs led to operating zones that significantly reduced the grid reliability during challenging conditions.

Moreover, considering the global climate changes that directly affect the grid operation in conjunction with the other consequences pointed by the 2018 Intergovernmental Panel on Climate Change (IPCC) report (IPCC, 2018), there is a need to significantly reduce the amount of anthropogenic greenhouse gases, such as the carbon dioxide, in the atmosphere. In this context, the environmental performance of energy system is a critical component as well, and thus should be considered as a separate objective.

A previous study has already acknowledged that multiple stakeholders should be considered during the dynamic optimization of energy system (Trifkovic et al., 2014). The multiple objectives were accounted by using a weighted sum of objectives into a single objective. The weights were chosen based on economic criteria. However, this MOO 
methodology does not guarantee that the optimal solution is a Pareto-optimal point. Another two common ways used to account the multiple objectives are either to adopt the economic objective as the main problem objective and constrain the environmental objective by an arbitrarily set value (Dowell and Shah, 2014), or to assess environmental performance by sensitivity analysis (Bankole et al., 2018). Neither of those pathways consider a methodology that aims to choose a Pareto-optimal compromise.

\subsection{Dynamic Real-time Optimization in PSE}

In the PSE field, the optimization is often performed to improve the steady-state behavior, considering that the system dynamics can be neglected. Under this hypothesis, the system can be described by a set of algebraic equations without time dependency. This assumption greatly simplifies the problem formulation, requiring less computational time when compared to the same problem size that accounts for time dependency. Also, the computational time is usually not a constraint as the optimal solution is not associated to time-dependent circumstances and valid for the steady-state operation.

However, the increasing need of improving process economics, efficiency, and product quality in a globalized market environment motivated the establishment of the real-time optimization (RTO) field which integrates economics and control performance. Often, the RTO is a linear or nonlinear steady-state optimization that calculates optimal setpoints, solved usually in time scale of hours to days. The RTO provides the optimal setpoints to a lower-level advanced controller, which in turn usually contains the system dynamic models (Tosukhowong et al., 2004; Camara et al., 2016; Jamaludin and Swartz, 2017; Li and Swartz, 2018).

One study pointed out that the main challenge of static RTO implementation is the level of uncertainty spread along the system, which often affect the determination of steadystate detection (Camara et al., 2016). Moreover, some systems such as the cycling of energy power plants will never operate in steady state due to the dynamic nature of their forcing functions and constraints. Therefore, to overcome the RTO steady-state assumption shortcomings, dynamics were introduced into the RTO layer, thus emerging the dynamic RTO field (Trifkovic et al., 2014; Pontes et al., 2015; Camara et al., 2016). 
When the DRTO is performed, an optimal trajectory is obtained as the optimization solution rather than an optimal setpoint.

A previous study performed a DRTO and control of a hybrid small-scale energy system which included solar energy, wind energy, battery, electrolyzers, hydrogen storage tanks, and fuel cells. The dynamic model was derived from mass and energy conservation equations and a day-ahead approach was developed. It was concluded that the DRTO layer added a proactive feature to the overall control architecture with high level of flexibility to integrate operational, economic and safety objectives (Trifkovic et al., 2014).

These recent studies (Trifkovic et al., 2014; Pontes et al., 2015; Li and Swartz, 2018) indicate that a DRTO layer has the potential to benefit the operation of large-scale industrial systems. However, the larger is the system, the more challenging becomes to obtain the optimal trajectories in real-time.

\subsubsection{Intersection of DRTO: Brief Review and Terminology Proposition}

In this subsection, a brief review is presented regarding the intersection of DRTO with different fields. Specifically, how the DRTO field was created and evolved with time, and the intersection of DRTO with the model predictive control (MPC) field. Moreover, a formal statement regarding the current interpretation of the DRTO nomenclature, i.e., the DRTO terminology is proposed.

As mentioned above, the RTO field was created to integrate economics and control layer. The classic RTO has a distinct two-level structure usually paired with a control level such as MPC, shown in Figure 2.2a. A recent modification of the structure is the RTO paired with distributed MPC architecture, as shown in Figure 2.2b. At the upper-level, plantwide RTO optimizes plant economics at low execution frequency (time scale of hours). Then, the RTO provides the set points or operation targets to the lower-level controllers. Typically, advanced multivariate control strategies are used to control and track set points at a higher execution frequency (time scale of minutes) (Jamaludin and Swartz, 2017). Although this strategy is applicable to some systems, other systems are not benefitted of such structure as they may never reach steady state due to presence of recycle loops, slow dynamics, or ramping needs. In those cases, the optimal RTO operating conditions may be suboptimal or even infeasible (Trifkovic et al., 2014). 
To overcome the RTO shortcomings due to the steady-state model, dynamics were introduced into the RTO layer to be performed at higher-level frequency, thus emerging the dynamic real-time optimization field (Pontes et al., 2015). Alternatively, economics were introduced into the MPC level, which lead to the field of economic MPC (EMPC) (Biegler, 2009; Angeli et al., 2012, Jamaludin and Swartz, 2017; Oyama and Durand, 2020), with some studies even completely eliminating or incorporating the RTO layer in the EMPC/MPC formulations (Biegler, 2009; Tran et al., 2014). Some of these modified strategies are shown in Figures 2.2c to 2.2e. A recent variation of the DRTO-MPC scheme is to mathematically incorporate the MPC necessary and sufficient Karush-Kuhn-Tucker optimality conditions into the DRTO optimization problem. The reformulation establishes a single-level mathematical program with complementarity constraints in a closed-loop manner (Jamaludin and Swartz, 2017; Li and Swartz, 2018), as shown in Figure 2.2f. Both MPC and DRTO strategies are constrained by their computational time to calculate the trajectories when implemented online.

With the computational and theoretical advances in the process optimization and control, the DRTO term, initially devised to indicate the use of dynamic models in the RTO level, has evolved. This term is sometimes used interchangeably to designate dynamic optimization and control strategies such as the MPC (and its variations) (Thierry and Biegler, 2019). Considering the broad optimization Formulation 2 presented in subsection 3.1.4, there is also no clear mathematical distinction between DRTO and MPC, as both are trying to minimize an objective subjected to dynamic models and constraints to obtain optimal trajectories, with the MPC also subjected to stability, observability, and controllability conditions. Currently, the main distinction between the DRTO and MPC is the time scale that they act upon, however with the same mathematical description. Therefore, this dissertation proposes the following statement to update and provide a structure to the broad DRTO term:

Proposition 1: MPC $\in$ DRTO, but DRTO $\notin$ MPC.

Under this proposition, the field of MPC intersects and it is contained in the DRTO field, but the field of DRTO intersects but it is not contained in MPC field as it also includes 
other areas of research, such as DRTO for dispatch and scheduling of energy systems (Trifkovic et al., 2014; Wang et al., 2020).

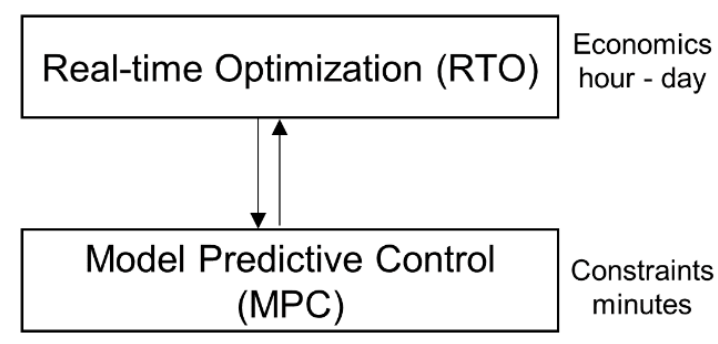

(a)

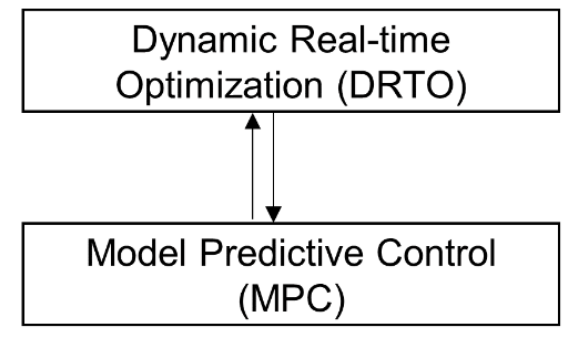

(c)

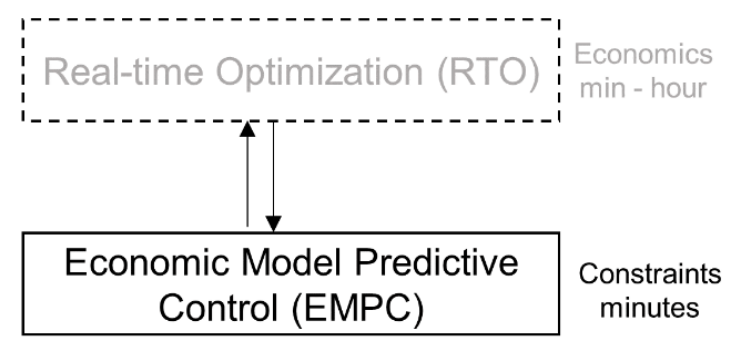

(e)

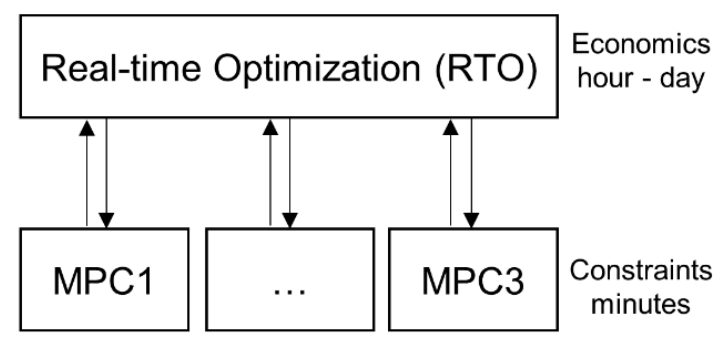

(b)

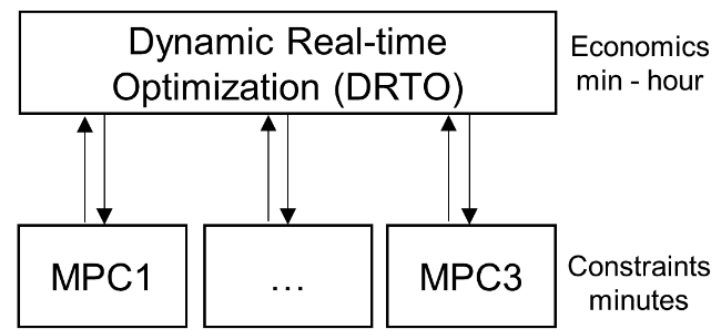

(d)

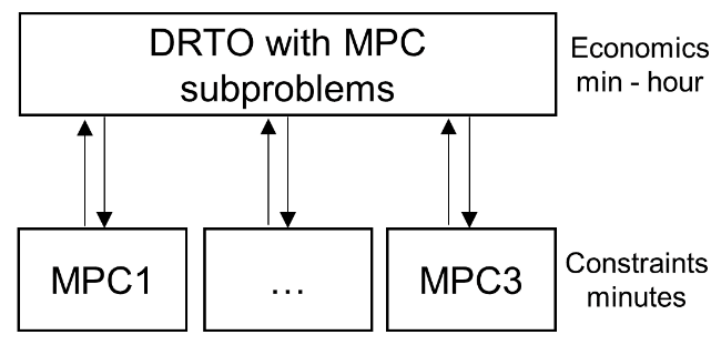

(f)

Figure 2.2. a) RTO paired with MPC, b) RTO paired with distributed MPC, c) DRTO paired with MPC, d) DRTO paired with distributed MPC, e) EMPC optionally pairing with RTO, f) DRTO with MPC subproblems paired with distributed MPC

\subsection{Literature Review Summary}

On the basis of the performed literature review, one of the main challenges of the cycling energy systems is to perform the optimization considering the systems dynamics and multi-objective nature of the problem in a timely manner. While some acknowledged the problem inherent multiplicity of objectives, very few studies incorporate a Pareto-based MOO technique to select the optimal compromise. In the context of coal-fired power 
plants with carbon capture system, selection of optimization time scale with respect to system dynamics is often not discussed. In the context of grid-wide operation, there is a knowledge gap regarding incorporating power plant health-related constraints during the dynamic dispatch of energy systems. The implementations of the developed NMPO and dynamic dispatch algorithms aim to fill these gaps and enable optimal operation of cycling baseload energy systems and optimal dispatch of energy system considering power plant health. 


\section{Optimization Approaches}

In this chapter, the general mathematical background and basic concepts for the proposed optimization frameworks are introduced. Specifically, the Nonlinear Multiobjective and dynamic real-time Predictive Optimization (NMPO) approach and pseudocodes are provided. The formulation of the MILP-based optimal dispatch is also presented.

3.1. Nonlinear Multi-objective and Dynamic Real-time Predictive Optimization (NMPO) Approach

The NMPO is composed of two main structures, the multi-objective optimization and the dynamic real-time optimization. Those structures are solved using two algorithms: the ParPSO and the Interior Point Optimization (IPOPT) (Wächter and Biegler, 2006). The following subsections are divided as follows: 1) ParPSO formulation; 2) IPOPT overview 3) MOO formulation; 4) DRTO formulation; and 5) Overall NMPO Algorithm.

\subsubsection{Parallelized Particle Swarm Optimization Formulation}

In this dissertation, the particle swarm optimization (PSO) is used to find a feasible IPOPT initial guess through learning and exploration of the decision variable space. The PSO is a meta-heuristic method inspired by the movements and communication within a group or swarm, such as insects or birds. Each particle that composes the swarm follows specific lower-level goals without knowledge of the higher-level goal. However, the lowerlevel goals express a meaningful collective behavior towards the higher-level goal. Each particle in the swarm is a potential solution and it is influenced by experiences of the neighboring particles as well as its own experience. Moreover, the global topology is adopted, i.e., each particle communicates with the entire swarm.

The PSO formulation considers how the particles move within a swarm and communicate to locate an optimum in the feasible space. Over time, through the combination of exploration and exploitation of past positions in the search space, the particles cluster or converge together around an optimum. Through exploration, the particles search new regions of the feasible space whereas through exploitation the particles attempt to improve or learn from the known promising regions. After the positions and velocities of 
each particle are initialized, the position of each particle in the next time step is updated recursively, as it is shown in Equations (3.1) and (3.2) (Sengupta et al., 2018):

$$
\begin{gathered}
v_{j}(t+1)=I T * v_{j}(t)+c_{1} * r_{1} *\left(P B_{j}-p_{j}(t)\right)+c_{2} * r_{2} *\left(S B-p_{j}(t)\right) \\
p_{j}(t+1)=p_{j}(t)+v_{j}(t+1)
\end{gathered}
$$

in which $p_{j}$ is the position of the $j$-th particle, $v_{j}$ is the velocity of the $j$-th particle, $P B_{j}$ is the personal best position of the j-th particle, $S B$ is the swarm best position, IT is the inertia term, $c_{1}$ and $c_{2}$ are the cognitive and social acceleration coefficients, and $r_{1}$ and $r_{2}$ are random numbers between $[0,1]$. The terms $I T, c_{1}$, and $c_{2}$ are determined using the constriction factor method. This method was developed from eigenvalue analysis of computational swarm dynamics and it is defined through Equations (3.3) to (3.6) (Sengupta et al., 2018):

$$
\begin{gathered}
I T=\frac{2 \gamma}{|2-\Omega-\sqrt{\Omega(\Omega-4)}|}, \quad \gamma \in[0,1] \\
\Omega=\Omega_{1}+\Omega_{2}, \quad \Omega \geq 4 \\
c_{1}=\operatorname{IT} * \Omega_{1} \\
c_{2}=\operatorname{IT} * \Omega_{2}
\end{gathered}
$$

in which $\Omega, \Omega_{1}, \Omega_{2}, \gamma$ are the constriction factors. Under this method, the swarm convergence is guaranteed with particles decelerating as iteration count increases. The constriction factor $\gamma$ controls the local or global convergence. When $\gamma$ is set close to 1 , particles traverse the search space with a predominant emphasis on exploration. When $\gamma$ is close to 0 the convergence is fast, but the solution quality may vary vastly. Then after a certain number of iterations, the swarm best is obtained. The particle velocities are also constrained at maximum equal to $35 \%$ of the difference of the decision variable's minimum and maximum. From an optimization perspective, the particle represents a potential solution or a trial point. The particle position is fully defined by a set of values within the decision domain $\mathbb{R}^{m}$, i.e., the position is a m-dimensional vector. The particle velocity determined by Equation (3.1) is also a m-dimensional vector that represents a change in the position value guided by the swarm dynamics, i.e., the update mechanism for the trial point. For instance, for a system with 2 inputs that can vary within the range of $[0,1]$, a particle position $\left(p_{1}\right)$ is fully defined as $p_{1}(t)=[0.2,0.3]$. In this case, $p_{1}$ is the 
potential solution. The swarm and personal bests are also $\mathrm{m}$-dimensional vectors updated according to the objective function. A schematic of the PSO update mechanism for one particle in a 2-dimensional space is shown in Figure 3.1.

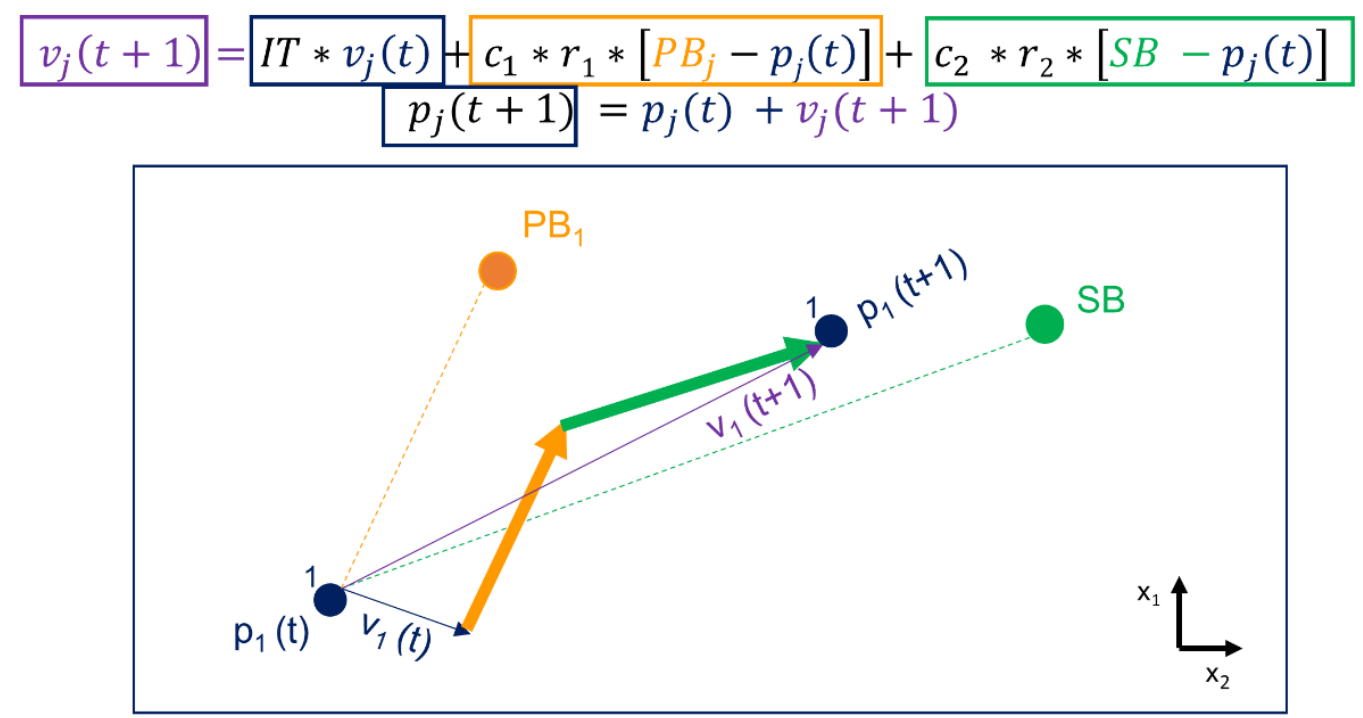

Figure 3.1. Schematic of the PSO update mechanism in 2-dimensional space However, as the number of decision variable increases, the computational time also increases and may lead to prohibitive computational time. Then to partially manage the computational time of a large-scale optimization problem and considering the overall decentralized nature of the PSO, the PSO method is parallelized. Parallel processing is a tool that can be used to efficiently solve non-gradient-based optimization problems and it may even improve the quality of the solutions as well as speed up the execution times (Venter and Sobieszczanski-Sobieski, 2005; Sola, 2010).

The parallelization in this dissertation is performed in MATLAB environment. MATLAB offers two way of parallelization: i) a single desktop system with local workers or multicore desktop workers; and ii) a computer cluster (Carrasco and Lima, 2018). The parallelization is performed in a single desktop system with 8 local cores (Inter ${ }^{\circledR}$ Xeon® CPU E5-1620v3, 3.5 GHz processor). The number of parallel workers should not be confused with the number of PSO particles. The number of particles and workers can be the same, but it is not a necessary condition. 
Currently, there are mainly two ways to perform the parallelization of the PSO: synchronously or asynchronously. In the synchronous parallelization, at each iteration every particle position and velocity are computed first and then the swarm best is updated. In the asynchronous parallelization approach, the PSO swarm best may be updated as information of new particles become available by the workers (Venter and Sobieszczanski-Sobieski, 2005).

The master and worker parallelization configuration is chosen as the parfor and cluster communication in MATLAB 2019 environment do not yet allow direct communication between workers. In the master and worker parallelization, one process is the master as it controls the execution of the other processes.

A test run was performed using the SCPC-CCS as the system with the asynchronous and synchronous parallelization configuration of the PSO. Considering the same number of particles and iterations, the asynchronous ParPSO often obtained a slightly better objective value, i.e., a lower objective value for a minimization formulation, than the synchronous parallelization. Regarding the computational time, the synchronous ParPSO however often finished computing the pre-determined iterations faster than the asynchronous ParPSO, mainly due to the coarse grain nature of the PSO formulation in MATLAB environment. In this environment, there is an imposed communication overhead between the master and the workers. Thus, considering that the optimality of the initial guess is not required and the real-time constraint of the optimization problem, the synchronous ParPSO was chosen to be implemented in the overall framework. Figure 3.2 shows the pseudocode with the main differences between the synchronous and asynchronous parallelizations of the PSO in MATLAB. Although the chosen platform and structure is not necessarily the most efficient way of parallelization (Venter and Sobieszczanski-Sobieski, 2005; Sola, 2010), it provides a lower bound in terms of how much this component could be sped-up in a single desktop parallelization. In this dissertation, an average of 4 -fold speed up is observed for the single desktop parallelization when compared to standard PSO formulation with the same number of particles and maximum iterations. 


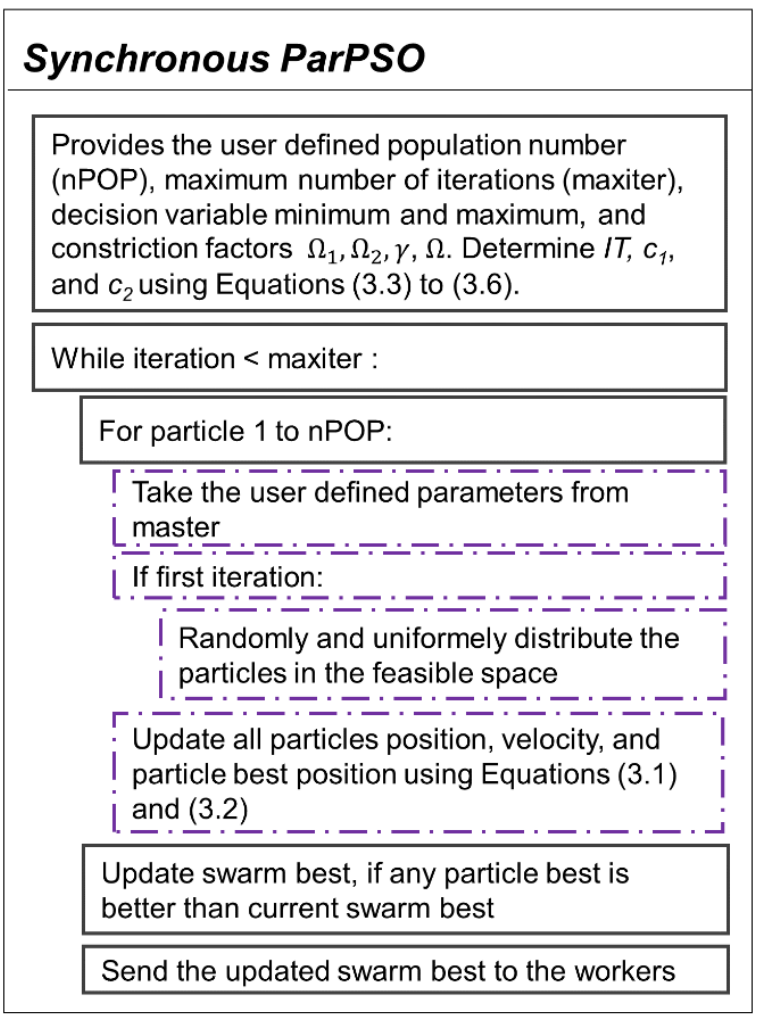

Master task

\section{Asynchronous ParPSO \\ Provides the user defined population number (nPOP), maximum number of iterations (maxiter), decision variable minimum and maximum, and constriction factors $\Omega_{1}, \Omega_{2}, \gamma, \Omega$. Determine $I T, c_{1}$, and $c_{2}$ using Equations (3.3) to (3.6).}

While iteration < maxiter :

For particle 1 to $\mathrm{nPOP}$ :

「 Take the user defined parameters from L master

If first iteration:

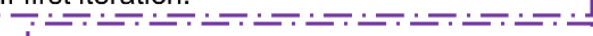

Randomly and uniformely distribute the i particles in the feasible space

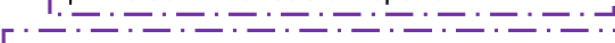

Update all particles position, velocity, and

I particle best position using Equations (3.1) and (3.2)

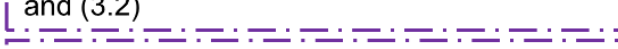

Check if particle best is better than swarm

best; if yes, send particle swarm information I to master

Send the updated swarm best to all the workers

Figure 3.2. Pseudocode of synchronous and asynchronous ParPSO approaches

\subsubsection{Interior Point Optimizer (IPOPT) Overview}

IPOPT is an open-source package designed to solve large-scale nonlinear optimization and it can solve general nonlinear programming problems structured as in Formulation 1:

\section{Formulation (1)}

$$
\min _{x \in X} \Phi(x)
$$

s.t.

$$
\begin{gathered}
c^{L B} \leq c(x) \leq c^{U B} \\
x^{L B} \leq x \leq x^{U B}
\end{gathered}
$$

in which, $\Phi$ is the objective function, $c$ the general nonlinear constraints, $x$ are the $\mathrm{m}$ dimensional decision variables, superscript LB stands for lower bound, and superscript UB stands for upper bound. 
IPOPT is a primal-dual interior-point algorithm with a filter line-search method that aims to find a local solution of large-scale nonlinear optimization problems. IPOPT has built in features such as inertia correction, second-order correction, and feasibility restoration phase that is activated if the trial step is not acceptable or matrix singularity is detected, which provides a robust optimization algorithm for large-scale nonlinear problems (Wächter and Biegler, 2006). IPOPT was developed for the AMPL modeling environment, but it has already been interfaced with Julia, GAMS, Python, C++, C, Java, Fortran, MATLAB, and many others through third parties and in-house packages (Vigerske and Wächter, 2021).

\subsubsection{Multi-objective Optimization Formulation}

The multi-objective or many objectives optimization problems is the simultaneous minimization (or maximization) of two or more conflicting objectives (Diwekar, 2008; Abouhawwash et al., 2020). A general mathematical definition is shown in Equation (3.7):

$$
\min _{x \in X} \Phi_{\mathrm{i}}(x), i=1, \ldots, n
$$

in which $\Phi_{\mathrm{i}}$ is the objective function to be minimized and subscript $n$ denotes the number of objective functions. The $\mathrm{MOO}$ can be defined as $\Phi: X \rightarrow Z$, in which the decision vector $x \in X$ in $\mathbb{R}^{m}$, and the $\mathrm{n}$-dimensional objective vector $\mathrm{z}=\Phi(x) \in \mathrm{Z}$ in $\mathbb{R}^{n}$.

In the MOO problems, the goal is no longer to find an optimal point, rather becomes the search of optimal tradeoffs. Specifically, Pareto-optimal compromise is defined using nondominance concepts (Diwekar, 2008). The dominance and non-dominance concepts are established in Definitions 1 to 4. Pareto set and front are further described in Definitions 5 and 6.

Definition 1: Solution $x_{1}$ dominates $x_{2} \Rightarrow \Phi_{\mathrm{i}}\left(x_{1}\right) \leq \Phi_{\mathrm{i}} \forall i=1, \ldots, n$ and $\exists i \in\{1, \ldots, k\}: \Phi_{\mathrm{i}}\left(x_{1}\right)<\Phi_{\mathrm{i}}\left(x_{2}\right)$.

Definition 2: If $\Phi_{\mathrm{i}}\left(x_{1}\right)<\Phi_{\mathrm{i}}\left(x_{2}\right)$, then $x_{1}$ strictly dominates $x_{2}$.

Definition 3: Solution $x_{3}$ is non-dominated Pareto optimal solution $\Rightarrow \nexists x_{4}: \Phi_{\mathrm{i}}\left(x_{4}\right)$ dominates $\Phi_{\mathrm{i}}\left(x_{3}\right) \forall i=1, \ldots, n$. 
Definition 4: If $\nexists x_{4}: \Phi_{\mathrm{i}}\left(x_{4}\right)$ strictly dominates $\Phi_{\mathrm{i}}\left(x_{3}\right) \forall i=1, \ldots, n$, then $x_{3}$ is weak Pareto optimal solution.

Definition 5: The Pareto set is the set of non-dominated Pareto optimal solutions.

Definition 6: The Pareto front is the set of corresponding objective values of the Pareto set.

In summary, $x_{1}$ is considered Pareto optimal if there exists no feasible decision vector set which would decrease some objective without causing a simultaneous increase in at least one other objective, in a minimization formulation. Pareto optimality is defined with respect to the entire decision variable space, unless otherwise specified. Thus, the Pareto optimal set is essentially a subset of a solution set and the evaluated objective vectors are the Pareto front, in which each vector is nondominated with respect to all objective vectors in the Pareto front (Coello et al., 2007).

Considering that the $\mathrm{MOO}$ is used in conjunction with a DRTO and the solutions need to be obtained within a time window, two methods are chosen to solve the MOO component, namely the modified Tchebycheff weighted metric method and the $\epsilon$-constraint method. Both methods allow to select a Pareto optimal solution, without generating the entire Pareto front, with minimal input from the decision maker.

The following subsections discuss two different ways to obtain weak Pareto optimal solutions with minimal interaction with the decision maker.

\subsubsection{Modified Tchebycheff Weighted Metric Method}

The weighted metric method decomposes the MOO problem into a set of scalar optimization problems. The general formulation of the weighted metric method is shown in Equation (3.8):

$$
\left.\left.\min _{x}\left(\sum_{i}^{n} w_{i} \mid \Phi_{i}(x)-u_{i}^{*}\right)\right|^{p}\right)^{1 / p}
$$

in which, $w$ is a n-dimensional weight vector with $\sum_{i=1}^{n} w_{i}=1$ and $w_{i} \geq 0, u^{*}$ is an ideal vector, and $p$ is a parameter between $[1, \infty]$. The ideal or utopian vector is further defined in Definition 7. 
Definition 7: The ideal or utopian point $u^{*} \in \mathbb{R}^{n}$ is defined as $\left\{u^{*} \in \mathbb{R}^{n}: \mathrm{u}_{i}^{*}=\min _{x} \Phi_{i}(x), i=1, \ldots, n\right\}$.

Figure 3.3 pictures the search mechanism for different values of $p$ for the weighted metric method. As pictured, for convex Pareto front, the compromises can be found with any $p$ value. For non-convex Pareto front, a common instance for nonlinear problems, part of the Pareto front compromises may not be available in the search space for low $p$ values. As the $p$ value increases, more compromises that are part of the non-convex Pareto front become available. Conversely, the higher is the $p$ value, the more non-smooth is the optimization formulation. At $p=\infty$, also known as the Tchebycheff weighted metric method, the minimization formulation in Equation (3.8) reduces to Equation (3.9).

$$
\min _{x} \operatorname{Tch}\left(\Phi(x) \mid w, u^{*}\right)=\max _{x}\left(w_{i}\left|\Phi_{i}(x)-u_{i}^{*}\right|\right)
$$

In the Tchebycheff weighted metric method, the optimal MOO compromise is determined by a specific weight vector and the distance of the chosen Pareto point to the ideal vector. From a determined initial point and specified weight vector, the optimizer iteratively minimizes the maximum distance until it reaches the compromise in the Pareto front and the objective cannot be further improved.

By inspection of Equation (3.9) and Figure 3.3 for $p=\infty$, one can infer that every Pareto point can be a solution of the Tchebycheff formulation by varying the weight vector. As a direct result, any Pareto optimal solution can be systematically found if the Tchebycheff metric is used, even for a non-convex Pareto-front. Therefore, any best compromise could be obtained in an online fashion, minimizing the interaction with the decision maker. 

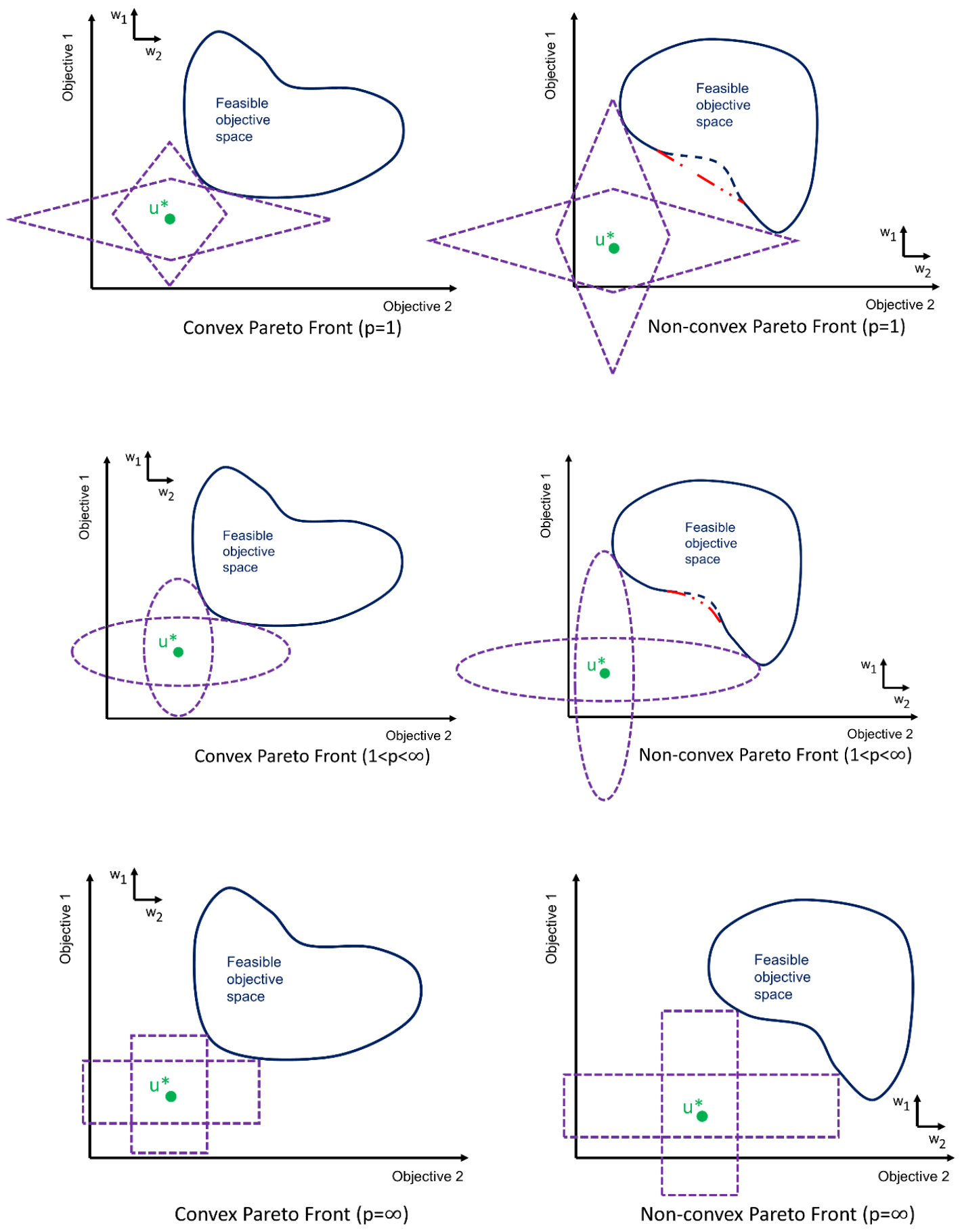

Figure 3.3. Weighted metric method search mechanism for different $p$ values (adapted from Narzisi, 2008) 
The solutions obtained by the conventional Tchebycheff decomposition method with uniform weights are not always uniformly distributed in the Pareto front. Therefore, the modified Tchebycheff (MTch) decomposition method was proposed (Ma et al., 2018) to address this issue. The modified decomposition is constructed as seen in Equation (3.10):

$$
\min _{x} \operatorname{MTch}\left(\Phi(x) \mid w, u^{*}\right)=\max _{1 \leq i \leq n}\left\{\frac{\Phi_{i}(x)-u_{i}^{*}}{w_{i}}\right\}
$$

Due to the property of Equation (3.10) of generating uniformly distributed solutions in the Pareto front when weights are uniformly changed, already reported in the open literature (Ma et al., 2018), the modified Tchebycheff method is adopted in this work when the weights are other than 0 .

As presented in Equation (3.9), a $w_{i}$ close to 1 has an intrinsic bias towards to the $i$-th objective. For the modified Tchebycheff as presented in Equation (3.10), a $w_{i}$ close to 0 has an intrinsic bias towards to the i-th objective. Thus, aiming to have a cohesive method that spans throughout the weight vector values in a continuous manner, including when the weight is equal to zero, a slight modification is proposed to Equation (3.9) to be used alongside Equation (3.10), as shown in Equation (3.11):

$$
\min _{x} \operatorname{Tch}\left(\Phi(x) \mid w, u^{*}\right)=\max _{1 \leq i \leq n}\left\{\left(1-w_{i}\right)\left|\Phi_{i}(x)-u_{i}^{*}\right|\right\}
$$

such that a $w_{i}$ close to 0 has an intrinsic bias towards to be closer to the i-th objective, as in the modified Tchebycheff method shown in Equation (3.10). The overall modified Tchebycheff-based method employed in this work is summarized below:

$$
\begin{array}{cc}
\min _{x} \operatorname{MTch}\left(\Phi(x) \mid w, u^{*}\right)=\max _{1 \leq i \leq n}\left\{\frac{\Phi_{i}(x)-u_{i}^{*}}{w_{i}}\right\} & \text { for } 0<w_{i}<1 \\
\min _{x} \operatorname{Tch}\left(\Phi(x) \mid w, u^{*}\right)=\max _{1 \leq i \leq n}\left\{\left(1-w_{i}\right)\left|\Phi_{i}(x)-u_{i}^{*}\right|\right\} & \text { for } w_{i}=0 ; w_{i}=1
\end{array}
$$

For both $T c h / M T c h$ formulations, it is recommended for the objectives to be normalized, which requires the objectives to be bounded and characterized.

Furthermore, as the MTch formulation is a minimization of a maximization, the optimization problem is not differentiable and non-smooth everywhere, only piecewise as the individual MTch distances are differentiable themselves. Therefore, the minmax 
optimization problem can be reformulated as a differentiable problem by introducing an extra variable $\alpha$ and $n$ nonlinear constraints, as shown in Equations (3.12) and (3.13):

$$
\min _{x \in X, \alpha \in \mathbb{R}^{-}}-\alpha
$$

s.t.

$$
\operatorname{MTch}_{i}+\alpha \leq 0, i=1, \ldots, n
$$

in which, $M T c h_{i}$ for $i=1, \ldots, n$ is the modified Tchebycheff indicator for $i$-th objective value, and $\alpha$ is the extra variable. In this formulation, the optimization is mathematically the same, the objective is linear, and the problem has differentiable objective function and constraints. It was chosen to maintain the $\alpha$ as a negative number as during the DRTO formulation all inequality constraints will be converted to equality constraints by introducing strictly positive slack variables to improve computational performance. Further explanation on the reformulation is provided in subsection 3.1.4.

\subsubsection{2. $\quad \epsilon$-constrained Method}

The $\epsilon$-constrained method is another way to solve a MOO problem by turning it into a set of single-objective optimization problems. In this method, one of the objectives is chosen (for instance $\left.\Phi_{1}\right)$ while the other objectives $\left(\Phi_{i}, i=2, \ldots, n\right)$ are turned into inequality constraints bounded by parametric values $\left(\epsilon_{i}, i=1, \ldots, n-1\right)$. In general terms, each individual optimization is solved, and $x_{i}$ solutions are determined. Then, corresponding values using the $x_{i}$ solution are calculated for the other objective, i.e., $\Phi_{i}\left(x_{k}\right), i=1, \ldots, n ; i \neq k$. The overall formulation is shown in Equations (3.14) to (3.16) (Diwekar, 2008):

$$
\begin{aligned}
& \min _{x} \Phi_{\mathrm{i}}(x) \\
& \epsilon_{i, \text { min }}^{L B} \leq \Phi_{\mathrm{i}} \leq \epsilon_{i, \text { min }}^{U B}, i=1, \ldots, n ; i \neq k \quad \text { for minimization } \\
& \epsilon_{i, \text { max }}^{L B} \leq \Phi_{\mathrm{i}} \leq \epsilon_{i, \text { max }}^{U B}, i=1, \ldots, n ; i \neq k \quad \text { for maximization }
\end{aligned}
$$

in which $\epsilon_{i}$ is the parametric value of the $\mathrm{i}$-th objective that it is not the k-th objective, subscript min is for minimization, and subscript max is for maximization. Theoretically, the objectives inequalities are bounded. The lower bound is their minimum value obtained by the individual single objective optimizations $(i=1, \ldots, n)$. The upper bound is the minimum 
corresponding value from the solution of the $k$-th single objective optimization $\left(\Phi_{i}\left(x_{k}\right), i=1, \ldots, n ; i \neq k\right)$ multiplied by a weight variable that represents the decision maker willingness to compromise. The bounds for the minimization and maximization formulations are shown in Equations (3.17) and (3.18).

$$
\begin{gathered}
\epsilon_{i, \text { max }}^{U B}=\epsilon_{i, \text { min }}^{L B}=\Phi_{i}\left(x_{i}\right), i=1, \ldots, n ; i \neq k \\
\epsilon_{i, \text { max }}^{L B}=\epsilon_{i, \text { min }}^{U B}=w_{\mathrm{i}}^{\epsilon-\text { const }} \Phi_{i}\left(x_{k}\right), i=1, \ldots, n ; i \neq k
\end{gathered}
$$

in which, $w_{\mathrm{i}}^{\epsilon-\text { const }}$ the weight vector for the e-constraint method and $w_{\mathrm{i}}^{\epsilon \text {-const }} \in[0,1]$. Although bounded, the only necessary constraints in the algorithm are the Equations (3.19) and (3.20) as these are the constraints that may be active during the optimization. Figure 3.4 pictures the search mechanism for the $\epsilon$-constrained method.

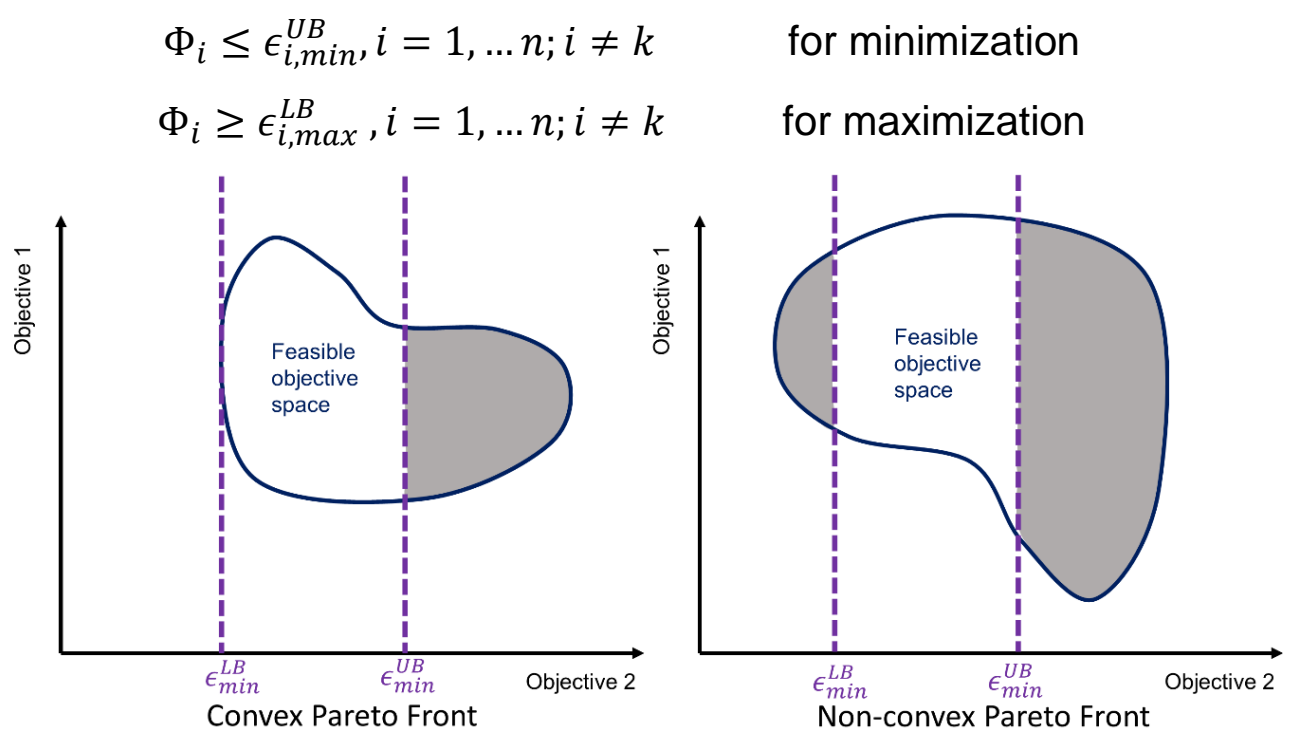

Figure 3.4. $\epsilon$-constrained method search mechanism (adapted from Narzisi, 2008) 


\subsubsection{Dynamic Real-time Optimization Formulation}

The general mathematical DRTO is defined in Formulation 2:

\section{Formulation (2)}

$$
\min _{x \in X} \Phi(x, t)
$$

s.t.

$$
\begin{gathered}
\frac{d x}{d t}=f(x, t) \\
h(x, t)=0 \\
g(x, t)<0
\end{gathered}
$$

in which, $f$ is the dynamic model that describes the system, $h$ are the equality constraints, $g$ are the inequality constraints, $x$ are the decision variables, and $t$ is the time.

As mentioned previously, the main concern while implementing a DRTO strategy in a large-scale system is the real-time constraint to obtain a solution. Furthermore, if the DRTO is extended with a MOO framework, both MOO problems would require optimization of every objective to be performed. For instance, for two objectives ( $\mathrm{i}=2$ ), the MTch MOO method solves $5(2 \mathrm{i}+1)$ DRTO problems: to find the minimum (i), the maximum (i), and the compromise (1). Whereas the $\epsilon$-constraint method performs at least $3(i+1)$ DRTO problems: to find the minimum (i) and the compromise (1).

The optimal trajectories are determined using dynamic programming considering the maximum principle and Bellman's principle of optimality. The principle of optimality states that the minimum or maximum value of a function is dependent on the initial state and the initial time (Diwekar, 2008). Therefore, the size of the optimization problem is associated to the optimization horizon. As the time horizon increases, so does the scale of the problem.

\section{Strategies to improve computational performance}

For gradient-based optimization, the slowest part of the algorithm tends to be the calculation of the first- and second-order derivatives. Besides the analytical way, derivatives can be obtained essentially in three ways: symbolic, numerical, or automatic. Symbolic differentiation is based on manipulating mathematical expressions to obtain the derivatives. If the problem is relatively simple or linear, symbolic differentiation can be 
used to obtain accurate derivatives. However, for a nonlinear problem or even a more complex framework, symbolic differentiation will hinder the computational time. Numerical differentiation determines approximation of derivatives using finite differences. However, this method can also be slow and there is a tradeoff between accuracy and employed step size. Automatic differentiation (AD) is based on the principle that every computer program is fundamentally a sequence of elementary arithmetic operations. The chain rule from calculus is then applied repeatedly to obtain any derivative order in automatic, efficient, and accurate manner. Therefore, the objective and constraint first-order derivatives are obtained using $A D$ techniques in this work. Without loss of generality, Formulation 2 is modified to Formulation 4 by adding slack variables $s$, as shown in Formulation 3 . Then the $x_{s}$ vector becomes the new decision variable vector and includes the $x$ decision variable vector and the slack variables.

Formulation (3)

$$
\min _{x \in X} \Phi(x, t)
$$

s.t.

$$
\begin{gathered}
h(x, t)=0 \\
g(x, t)+s=0 \\
s \geq 0
\end{gathered}
$$

Formulation (4)

$$
\min _{x_{s} \in X} \Phi\left(x_{s}, t\right)
$$

s.t.

$$
\begin{gathered}
h_{s}\left(x_{s}, t\right)=0 \\
x_{s}=[x, s]
\end{gathered}
$$

From Formulation 4, Automatic Differentiation for MATLAB (ADiMAT) (Bischof et al., 2002) package is used to obtain the objectives $(\Phi)$ and constraint $\left(h_{s}\right)$ first-order derivatives $\left(\nabla \Phi, \nabla h_{s}\right)$. Then those derivatives are provided to the optimization algorithm used to solve the DRTO problems, the Interior Point Optimizer (IPOPT). In this dissertation, considering the same tolerances, speed up within the range of 80 to 120 times faster is observed when compared to the default IPOPT finite differences. Regarding the second-order derivatives, IPOPT uses the Broyden-Fletcher-Goldfarb-Shanno (BFGS) algorithm to update the Hessian. IPOPT was connected to MATLAB using the open-source OPTI toolbox (Currie and Wilson, 2012). Note that by adding the slack variables, the size of the problem is increased.

IPOPT performance can also be hindered if the scale of the decision variables, dependent variables, and objective values vary vastly. Improvement of computational time is 
observed after the normalization of decision and dependent variables as well as the objective functions. Finally, as shown in Figure 1.2, linear and nonlinear reduced-order models of high-fidelity Aspen Plus Dynamics first-principles models can also be generated for online implementation purposes.

\subsubsection{Overall NMPO Algorithm}

The proposed approach to optimize large-scale process under cycling and different objectives is the Nonlinear Multi-objective and dynamic real-time Predictive Optimization (NMPO), solved with ParPSO and IPOPT algorithms. The ParPSO finds a feasible initial guess with respect to the trajectory for the IPOPT. The ParPSO also explores and learns from the available feasible space. Then, the feasible guess is used to seek optimality with respect to the whole trajectory in the DRTO solved using IPOPT and ADiMAT. At last, the individual optimal trajectories are used in the MOO component to find the optimal compromise, also solved using IPOPT.

The proposed execution of the overall NMPO algorithm is shown in Figure 3.5. This architecture provides prediction capabilities and time for the algorithm to act upon the forcing function information as they become available. Forcing function is considered here as any function/variable that can influence the optimization solution that is not a decision variable for the optimizer, such as electricity price and demand for the current application. Specifically, the algorithm has 2 horizons: the optimization and the implementation time horizons. At first, the optimizer generates a trajectory with respect to the optimization time horizon, but only the trajectory up to the implementation horizon is applied to the system. After the implementation, the forcing function predictions information can be updated, and the trajectories are recalculated. The overall framework is then implemented in a rolling horizon manner. Therefore, the calculation time horizon of the trajectories must be shorter than the implementation horizon.

Therefore, the NMPO approach aims to address a gap that has not yet been tackled by existing methods. The approach incorporates a MOO method that searches Paretooptimal compromises by design using either the Modified Tchebycheff or the $\epsilon$-constraint methods. The NMPO also incorporates strategies to improve computational performances such as $A D$ and parallelization. Additionally, the NMPO updates the 
forecast of the forcing functions as time evolves. The NMPO is one of the first efforts that incorporates all these elements (MOO, DRTO, and computational tractability) to address the cycling of baseload power plants under VRE penetration.

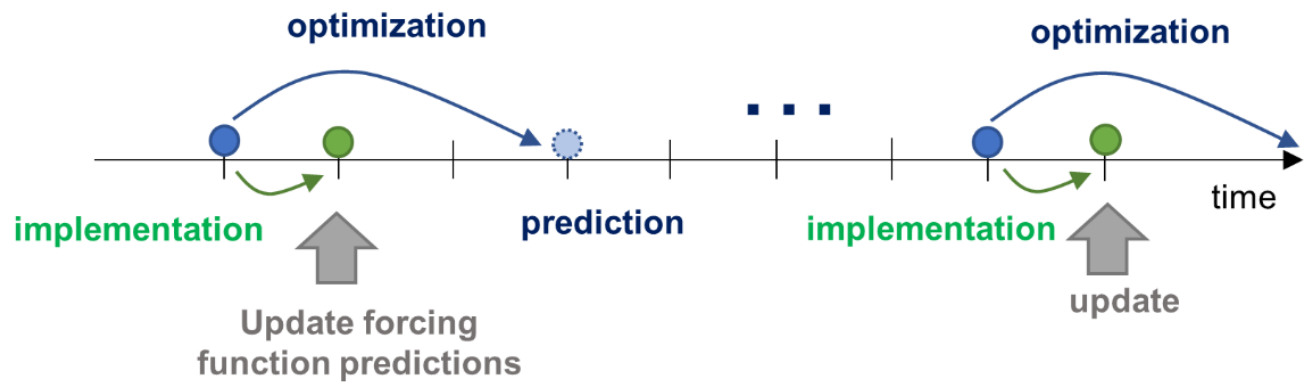

Figure 3.5. Schematic of proposed optimization framework implementation

Figure 3.6 outlines the proposed NMPO algorithm for baseload systems under cycling conditions that consists of the seven steps described below:

Initialization: The dynamic system model and forcing functions, the optimization and implementation time horizons, the ParPSO parameters, and the weight vector determined by the decision maker are sent to the optimizer to initialize the overall algorithm.

(i) The time is set to $t=t_{0}$, which refers to the initial time of the NMPO dynamic programming.

(ii) The MATLAB parallel environment is initialized with 8 workers (1 worker/core) in a synchronous architecture. The ParPSO particles are created, randomly and uniformly distributed in the feasible space. Random velocities are also attributed for each particle. The swarm best position thus far is determined from their randomly assigned initialization.

(iii) After initialization, the position and velocities are updated recursively for a maximum number (maxiter) of iterations. At the end of the iterations, the feasible solution for the optimization horizon is sent to IPOPT as initial guess. The ParPSO step is terminated after a predetermined number of iterations is reached.

(iv) The DRTO problem derivatives are obtained using ADiMAT. Then the optimal trajectories up to time $t_{2}$, maximum, and minimum are calculated for each objective using 
IPOPT connected to MATLAB through the OPTI toolbox. The DRTO/IPOPT step is terminated after the set dual infeasibility and complementarity tolerances are satisfied.

(v) The MOO problem derivatives are obtained using ADiMAT. Then, either the $\epsilon$-constraint or the Tchebycheff-based $\mathrm{MOO}$ method is used to formulate the optimization problem. The optimal compromise is chosen considering the weight vector. The MOO/IPOPT step is terminated after the set dual infeasibility and complementarity tolerances are satisfied.

(vi) After the trajectories for the optimization horizon are determined, such trajectories are partially implemented considering the implementation horizon $t_{1}$ and the time horizon is updated as $t=t+t_{1}$.

(vii) Assessment if NMPO has reached $t=T$ :

1. If yes, exit the optimizer framework.

2. If no, then repeat steps (ii)-(vii) until the criterion $t=T$ is satisfied. 


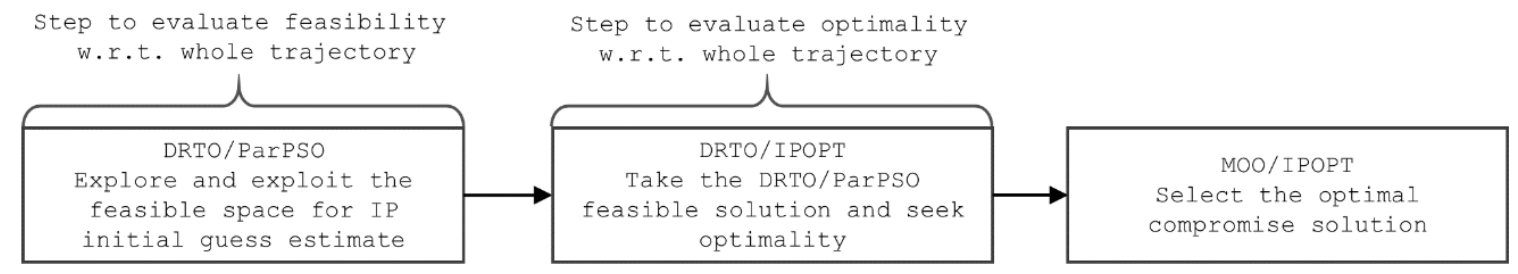

(i)

\section{START}

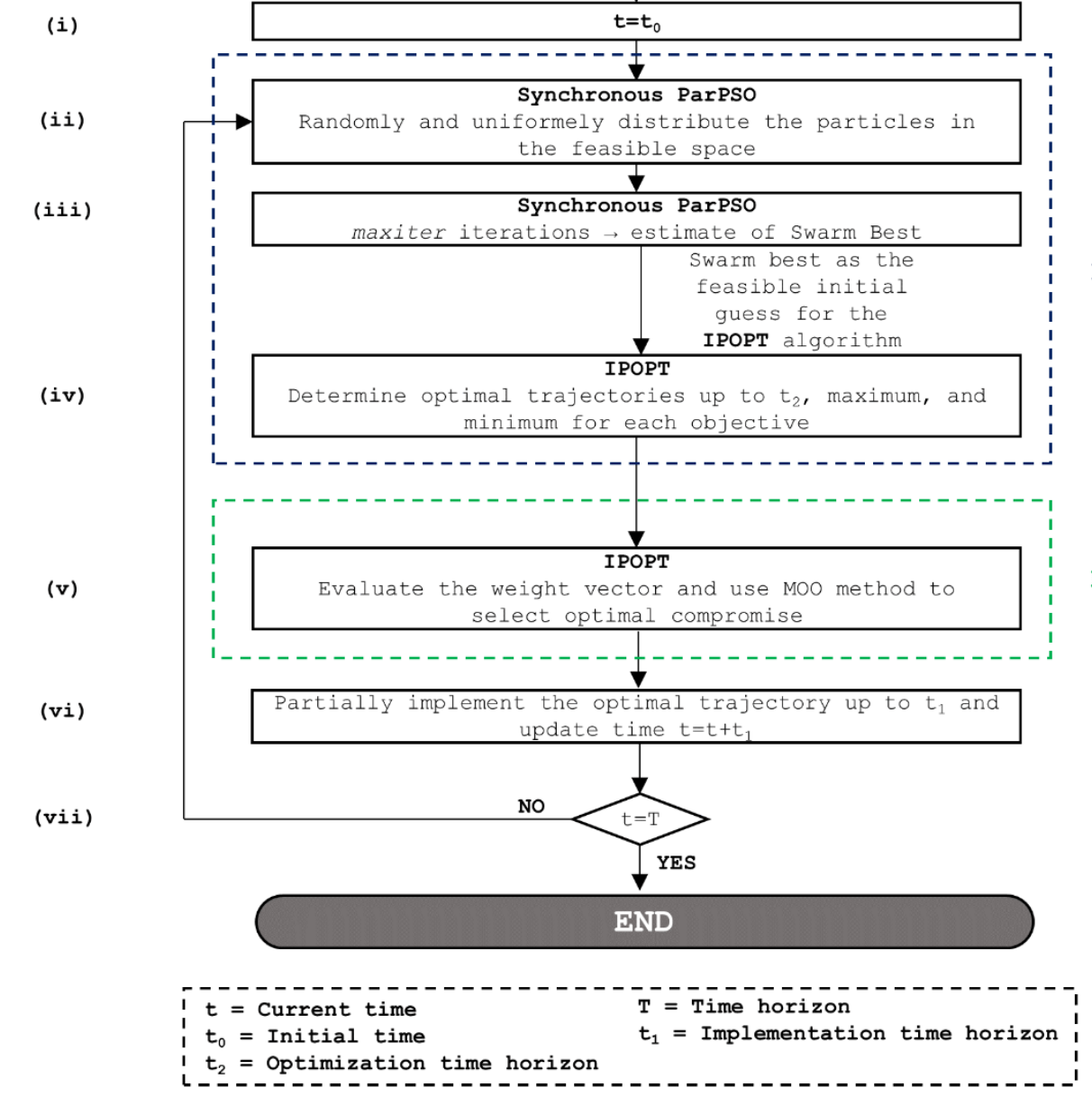

Figure 3.6. Pseudocode for the implementation of the NMPO framework

\subsection{MILP-based Approach for Dynamic Dispatch of Energy Systems Considering Power Plant Health}

The mixed-integer linear programming (MILP) is employed when some of the decision variables are constrained to be an integer value at the optimal solution. The integer can be any value from the $\mathbb{Z}$ set. Often, to model Boolean constraints, the integer is binary 
$\{0,1\}$, which represents TRUE or FALSE alternatives. Then the constraints are formulated employing the disjunction operators (and, or) for logical propositions (Morales-España et al., 2015).

In this dissertation, the focus of the MILP formulation is to perform a 6-minute ahead dispatch for 30 days that responds to demand and variable renewable deviation while considering the dynamic models of the energy systems and power plant health-related constraints. The integer variables are introduced to include a logical constraint regarding system operation during optimization. Considering the goal of this algorithm, Formulation 2 can be adapted to incorporate integer variables as shown in Formulation 5:

\section{Formulation (5)}

$$
\min _{x \in X} \Phi(x, v, t)
$$

s.t.

$$
\begin{gathered}
\frac{d x}{d t}=f(x, v, t) \\
h(x, v)=0 \\
g(x, v) \leq 0 \\
x \in X, v \in \mathbb{Z}
\end{gathered}
$$

in which $v$ are the integer variables, i.e., $v \in \mathbb{Z}$. This same framework can be extended to perform a unit commitment problem in which each energy system is represented by a set of binary variables, as already performed in literature (Morales-España et al., 2015).

Furthermore, the models are developed in MATLAB and the optimization algorithm is solved using branch and bound in the intlinprog subroutine. The models used in the dynamic dispatch are linear reduced-order models of the high-fidelity Aspen Plus Dynamic first-principles models. A perfect demand forecast of at least 6 min ahead of time is assumed.

The overall framework could be adapted to incorporate the most updated forcing function data accordingly. The energy systems are assumed to be always committed at this level by an independent scheduler; thus, shutdown/startup procedures are not considered during the dispatch. The specific power health related constraints are based on NGCC mechanical and thermal stresses and they are further defined in Chapter 5. 


\section{Implementation of NMPO Approach}

In this chapter, the details of the NMPO implementation are provided. Specifically, a brief description of the SCPC-CCS system and the method used to reduce the order of the high-fidelity SCPC-CCS first-principles model are performed. Then the nonlinear optimization results are presented.

4.1. Supercritical Pulverized Coal-fired Power Plant with MEA-based Carbon Capture (SCPC-CCS) Dynamic Modeling

The application used in the NMPO framework is the SCPC with monoethanolamine (MEA)-based carbon capture system (SCPC-CCS), for which a simplified diagram is shown in Figure 4.1. The SCPC-CCS is designed to operate in baseload conditions, i.e., for a constant power output. The dynamic model was built in Aspen Plus Dynamics (Zhang et al., 2016; He and Lima, 2020) and it was based on the Cases 12A/12B of the 2015 NETL report (NETL, 2015). The SCPC net capacity is 550MWe and uses a single $24.1 \mathrm{MPa} / 593^{\circ} \mathrm{C} / 593^{\circ} \mathrm{C}$ superheat/reheat cycle. The SCPC-CCS can be subdivided in three main sections: (i) feed processing; (ii) emission control units; and (iii) combined cycle power generation.

In the feed processing, the air is preheated and conditioned before it is fed with coal into the boiler. Then the generated heat in the boiler produces the steam for power generation. The boiler flue gas stream is sent to the pollution control section.

In the emission control, the particulate of the flue gas is removed by the bag house. The flue gas is driven by induced draft fan to the desulfurization unit, where sulfur and NOx are removed from the flue gas. Afterwards, the flue gas is forwarded to the carbon capture system (CCS), where the flue gas is initially conditioned through a set of pumps and direct contact cooler (DCC) to suitable pressure and temperature to enter in the absorber. After the conditioning, the flue gas is sent to the absorber where the $\mathrm{CO}_{2}$ is absorbed into the MEA solvent (lean solvent) and the cleaner flue gas is vented to the atmosphere with lower $\mathrm{CO}_{2}$ content. The MEA solvent rich in $\mathrm{CO}_{2}$ then goes to the stripper where the solvent is regenerated and lean MEA solvent is recycled back to the absorber. The 
resulting high-purity $\mathrm{CO}_{2}$ can be stored or conditioned for other purposes, by pressurizing it using a multistage compressor.

The energy penalty for $\mathrm{CO}_{2}$ removal is significant due to the multistage compressors and the required thermal energy to regenerate the solvent in the stripper reboiler. Specifically, the thermal energy for solvent recovery is obtained by extracting low-pressure steam from the power plant.

Finally, in the combined cycle power generation, the superheated steam coming from the boiler passes through high-,intermediate-, and low-pressure turbines. After the intermediate-pressure stage, part of the low-pressure steam is extracted and provided to the CCS to regenerate the solvent in the stripper reboiler. Before returning to the boiler, the boiler feedwater is heated by a series of integrated heat recovery subsystem (NETL, 2015; Zhang et al., 2016; He and Lima, 2020).

For dynamic optimization purposes, the high-fidelity first-principles model is simplified for computational tractability. There are some toolboxes available that facilitate the generation of reduced-order models. For instance, Dynamic Reduced Models (D-RM ${ }^{\mathrm{TM}}$ ) Builder is a toolbox that generates dynamic reduced-order models based on the structures of decoupled $\mathrm{A}-\mathrm{B}$ net and the nonlinear autoregressive moving average models. The D-RM ${ }^{\mathrm{TM}}$ can be directly connected to Aspen Custom Modeler or generate the reduced models from user-provided data (Ma et al., 2016; CCSI, 2018). The MATLAB System Identification ${ }^{\mathrm{TM}}$ is another toolbox that generates linear and nonlinear dynamic reduced models from user-provided data, with connectivity to Simulink. In this toolbox, there is an array of linear model structures, such as linear autoregressive with exogenous inputs (ARX), autoregressive moving average with exogenous inputs, state-space models, transfer functions, and many others. Regarding the nonlinear models, the structures are nonlinear ARX, Hammerstein-Wiener, and grey-box models. 


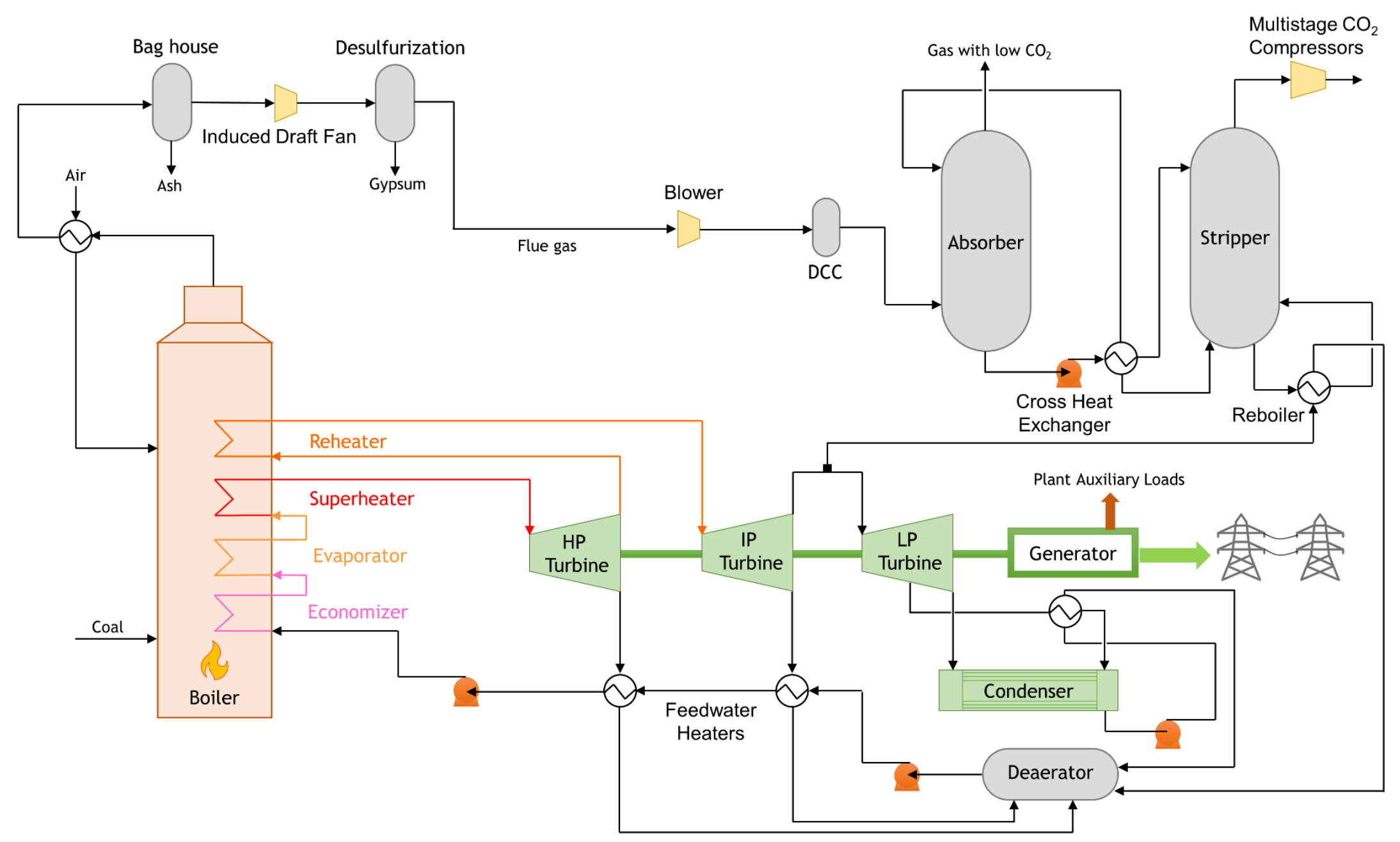

Figure 4.1. Supercritical coal-fired power plant with the MEA-based carbon capture 
In this dissertation, as the SCPC-MEA power plant input/output relationship is a mix of linear and nonlinear behaviors and the optimization is in MATLAB environment, the MATLAB System Identification ${ }^{\mathrm{TM}}$ Toolbox is chosen to generate the reduced-order models seamlessly. In particular, the linear and nonlinear AutoRegressive with eXogenous inputs (ARX/NARX) models, with wavelet networks are selected to represent the nonlinearities with standard and custom regressors.

The general input-output relationship given by a NARX structure is described by the nonlinear Equation (4.1) below:

$$
y(t)=f c\left(y(t-1), \ldots, y\left(t-n_{y}\right), x(t), x(t-1), \ldots, x\left(t-n_{x}\right)\right)+\varepsilon(t)
$$

in which $f c$ is a nonlinear mapping, $y$ are the dependent variables, $\varepsilon$ is an independent identically distributed random variable, and $n_{x}$ and $n_{y}$ are the maximum input and output lags, respectively (Wei et al., 2003). It is assumed that the nonlinear mapping $f c$ can be characterized as a finite set of hierarchical correlated functions expanded in terms of the lagged output and input variables. Equations (4.2) to (4.5) show the general structure of the wavelet network:

$$
\begin{gathered}
\chi=\left[y(t-1), \ldots, y\left(t-n_{y}\right), x(t), x(t-1), \ldots, x\left(t-n_{u}\right)\right] \\
f c(\chi)=(\chi-r) P L+a s_{1} \times \theta\left(b s_{1}(\chi-r) Q-c s_{1}\right)+\cdots \\
+a s_{n_{s}} \times \theta\left(b s_{n_{s}}(\chi-r) Q-c s_{n_{s}}\right)+a w_{1} \times \psi\left(b w(\chi-r) Q-c w_{1}\right) \\
\left.+\cdots+a w_{n_{w}} \times \psi\left(b w_{n_{w}}(\chi-r) Q-c_{n_{w}}\right)\right)+d \\
\theta(\chi)=\exp \left(-0.5 \chi^{T} \chi\right) \\
\psi(\chi)=\left(\operatorname{dim}(\chi)-\chi^{T} \chi\right) \times \exp \left(-0.5 \chi^{T} \chi\right)
\end{gathered}
$$

in which, $\chi$ is a I-dimensional vector of regressors, $Q$ and $P$ are I-by-q and I-by-p nonlinear and linear projection matrices determined by the principal component analysis of estimation data, the 1-by-I vector $r$ is the mean of $\chi$ computed from the estimation data. Also, $n_{s}$ and $n_{w}$ are the number of scaling and wavelet units, $L$ is a p-by- 1 linear coefficient, as is ns-by-1 scaling coefficient vector, aw is a nw-by-1 wavelet coefficient vector, $b s$ is a ns-by-1 scaling dilation vector, bw is a nw-by- 1 wavalet dilation vector, cs is a ns-by-q scaling translation matrix, $c w$ is a nw-by-q wavelet translation matrix, and $d$ is the output offset scalar. The $\theta$ and $\psi$ are the scaling and wavelet functions, respectively (Mathworks, 2021). 
Regarding the input-output ARX relationship, the general structure is described by Equation (4.6) below:

$$
\begin{aligned}
& y(t)+a_{1} y(t-1)+\cdots a_{n_{a}} y\left(t-n_{a}\right) \\
& \quad=b_{1} x\left(t-n_{k}\right)+\cdots+b_{n_{b}} x\left(t-n_{b}-n_{k}+1\right)+w n(t)
\end{aligned}
$$

in which $n_{a}$ is the number of poles, $n_{b}$ is the number of zeros, $n_{k}$ is the deadtime of the system, also known as the delay, $a_{1}, \ldots, a_{n_{a}}$ and $b_{1}, \ldots, n_{b}$ are the regression coefficients, and $w n(t)$ is the white noise at time $t$.

The input or decision variables and the output or dependent variables for the SCPC-CCS power plant are shown in Table 4.1. The CCS equivalent work is assumed as the total power required for the CCS to operate and it is the sum of to the power supplied to the blowers and compressors as well as the equivalent work associated with the thermal energy supplied to the reboiler of the stripper. Therefore, the dimensions of input $x$ output variables are currently $7 \times 10$.

Table 4.1. Decision and dependent variables for the SCPC-CCS system

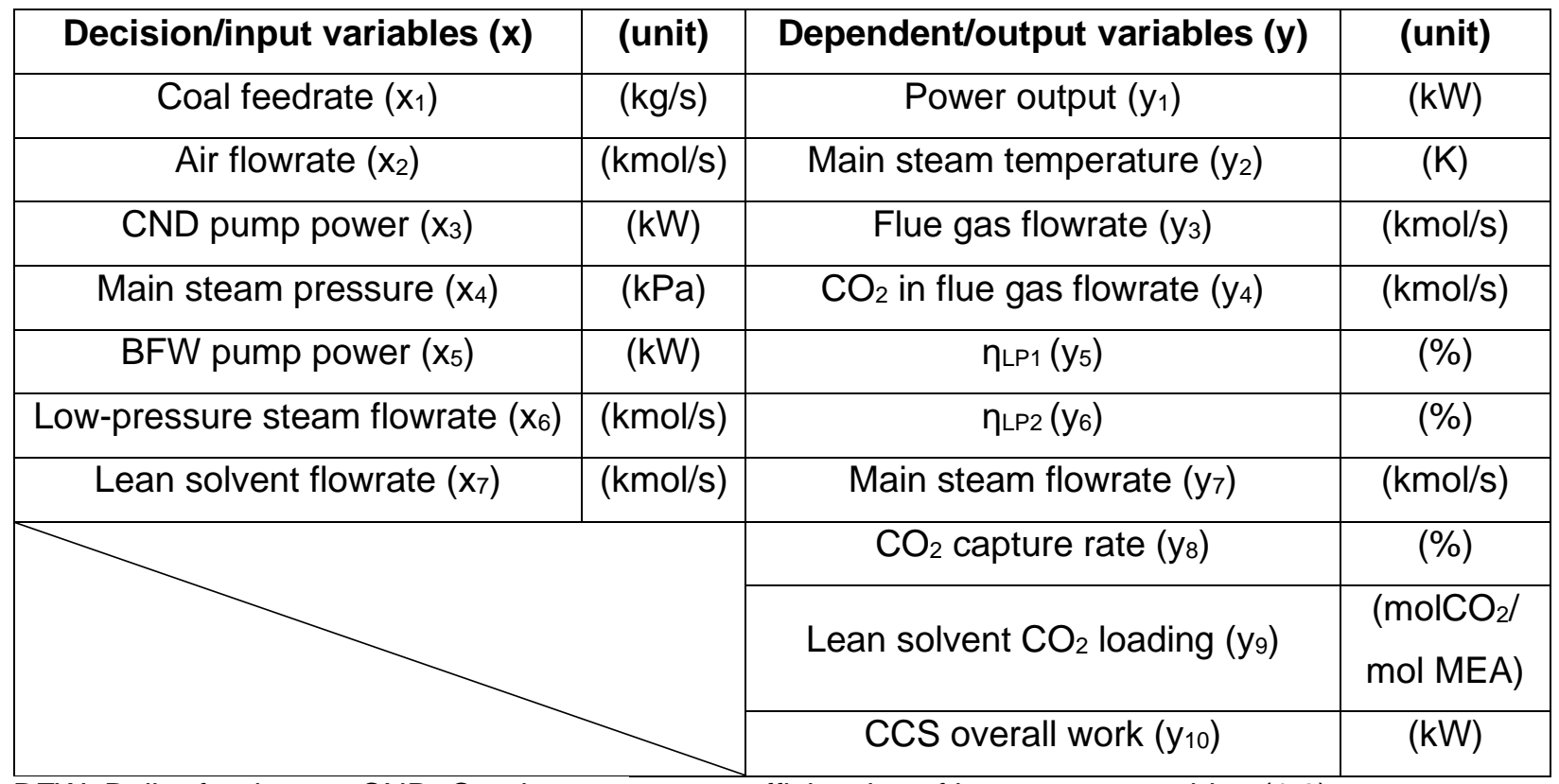

BFW: Boiler feedwater; CND: Condenser; १LP1,LP2: efficiencies of low-pressure turbine $(1,2)$.

Standard and custom regressors are used to predict the dependent variables value, as shown in Table 4.2. The data used for system identification is generated from the high- 
fidelity first-principles model. The designed sequence to excite the decision variables is based on pseudo-random binary signals (PRBS).

The goodness of fit (GOF) standard used to determine the correspondence between the 1-hour ahead prediction with the data from the high-fidelity model in Aspen® Dynamics is defined in Equations (4.7) and (4.8). The standard is defined based on the normalized root mean squared error (NRMSE) (Ljung, 2021).

$$
\begin{aligned}
& \text { NMRSE }=\frac{\left\|y_{\text {data }}-\hat{y}_{\text {prediction }}\right\|}{\left\|y_{\text {data }}-\bar{y}_{\text {data }}\right\|} \\
& \text { GOF }=(1-N M R S E) \times 100 \%
\end{aligned}
$$

In which, $y_{\text {data }}$ is the dependent/output data from the high-fidelity model, $\hat{y}_{\text {prediction }}$ is the dependent/output prediction by the reduced-order model, and $\bar{y}_{\text {data }}$ is the average of the dependent/output data from the high-fidelity model. The NMRSE is recommended to be used when comparing different models. The GOF can vary between $-\infty$ to $100 \%$. A GOF value equal to 0 indicates that the ROM model is as good at fitting as a constant line equal to the data mean. A GOF value equal to $100 \%$ indicates a perfect fit (Ljung, 2021). The ROM is chosen based in the GOF values, focusing mainly on the predictions of power output $\left(G O F\left(y_{1}\right)=91.13 \%\right)$ and the $\mathrm{CO}_{2}$ capture rate $\left(G O F\left(y_{8}\right)=91.61 \%\right)$.

Particularly, due to the deadtime of approximately 30 minutes in the response of the CCS to varying inputs, the PRBS test is designed to introduce step tests for the low-pressure steam, $\mathrm{CO}_{2}$ in flue gas, and lean solvent flowrates, i.e., the decision variables of the CCS, every 1 hour and the responses of the CCS dependent variables ( $y_{8}$ to $\left.y_{10}\right)$ are recorded. As a mathematical way of representing this behavior in the ROM, cosine and sine functions are used as regressors for the CCS dependent variables $\left(y_{8}\right.$ to $\left.y_{10}\right)$.As the dynamics of the feed processing and generation are faster, the PRBS has introduced steps in the coal feedrate, air flowrate, condenser pump power, main steam pressure, and boiler feedwater pump power input variables every 15 minutes. Particularly, the flue flowrate $\left(y_{3}\right)$ is measured after the desulfurization process. 
Table 4.2. Regressors of the linear and nonlinear ARX models for SCPC-CCS and correspondence between data and 1-hour ahead predictions

\begin{tabular}{|c|c|c|c|}
\hline $\begin{array}{c}\text { Dependent } \\
\text { variable }\end{array}$ & Custom regressors & Standard regressors & GOF (\%) \\
\hline $\mathrm{y}_{1}$ & $\begin{array}{c}\mathrm{x}_{1}(\mathrm{t}-1) \times \mathrm{x}_{5}(\mathrm{t}-1) ; \mathrm{x}_{1}(\mathrm{t}) \times \mathrm{x}_{5}(\mathrm{t}) \\
\mathrm{x}_{1}(\mathrm{t}) \times \mathrm{x}_{1}(\mathrm{t}-1) ; \mathrm{x}_{5}(\mathrm{t})^{2} \\
\mathrm{x}_{5}(\mathrm{t}-1)^{2}\end{array}$ & $\begin{array}{l}\mathrm{y}_{1}(\mathrm{t}-1, \mathrm{t}-2, \mathrm{t}-3) ; \mathrm{x}_{1}(\mathrm{t}, \mathrm{t}-1, \mathrm{t}-2) \\
\mathrm{x}_{3}(\mathrm{t}, \mathrm{t}-1, \mathrm{t}-2) ; \mathrm{x}_{4}(\mathrm{t}, \mathrm{t}-1, \mathrm{t}-2) \\
\left.\mathrm{x}_{5}(\mathrm{t}, \mathrm{t}-1, \mathrm{t}-2)\right) ; \mathrm{x}_{6}(\mathrm{t}, \mathrm{t}-1, \mathrm{t}-2)\end{array}$ & $91.13 \%$ \\
\hline $\mathrm{y}_{2}$ & $\mathrm{x}_{1}(\mathrm{t}, \mathrm{t}-1) ; \mathrm{y}_{2}(\mathrm{t}-1)^{2}$ & $\begin{array}{c}\mathrm{y}_{2}(\mathrm{t}-1, \mathrm{t}-2, \mathrm{t}-3) ; \mathrm{x}_{4}(\mathrm{t}) \\
\mathrm{x}_{5}(\mathrm{t}, \mathrm{t}-1)\end{array}$ & $86.98 \%$ \\
\hline $\mathrm{y}_{3}$ & $\begin{array}{l}\mathrm{x}_{1}(\mathrm{t})^{2} ; \mathrm{x}_{2}(\mathrm{t})^{2} \\
\mathrm{x}_{1}(\mathrm{t}) \times \mathrm{x}_{1}(\mathrm{t}-1)\end{array}$ & $\begin{array}{c}\mathrm{y}_{3}(\mathrm{t}-1, \mathrm{t}-2, \mathrm{t}-3) ; \mathrm{x}_{1}(\mathrm{t}, \mathrm{t}-1, \mathrm{t}-2, \mathrm{t}-3) \\
\mathrm{x}_{2}(\mathrm{t}, \mathrm{t}-1,-2, \mathrm{t}-3) ; \mathrm{x}_{4}(\mathrm{t}, \mathrm{t}-1, \mathrm{t}-2)\end{array}$ & $70.45 \%$ \\
\hline $\mathrm{y}_{4}$ & $\mathrm{y}_{4}(\mathrm{t}-1) ; \mathrm{x}_{1}(\mathrm{t})$ & - & $92.43 \%$ \\
\hline $\mathrm{y}_{5}$ & $\begin{array}{c}\mathrm{x}_{5}(\mathrm{t})^{2} ; \mathrm{x}_{5}(\mathrm{t}-1)^{2} \\
\mathrm{x}_{5}(\mathrm{t}) \times \mathrm{x}_{5}(\mathrm{t}-1)\end{array}$ & $\mathrm{y}_{5}(\mathrm{t}-1, \mathrm{t}-2, \mathrm{t}-3) ; \mathrm{x}_{5}(\mathrm{t}, \mathrm{t}-1, \mathrm{t}-2)$ & $75.79 \%$ \\
\hline $\mathrm{y}_{6}$ & $\begin{array}{c}\mathrm{x}_{5}(\mathrm{t})^{2} ; \mathrm{x}_{5}(\mathrm{t}-1)^{2} \\
\mathrm{x}_{5}(\mathrm{t}) \times \mathrm{x}_{5}(\mathrm{t}-1)\end{array}$ & $\mathrm{y}_{6}(\mathrm{t}-1, \mathrm{t}-2, \mathrm{t}-3) ; \mathrm{x}_{5}(\mathrm{t}, \mathrm{t}-1, \mathrm{t}-2)$ & $74.13 \%$ \\
\hline$y_{7}$ & $\begin{array}{c}\mathrm{y}_{7}(\mathrm{t}-1, \mathrm{t}-2) ; \mathrm{x}_{1}(\mathrm{t}, \mathrm{t}-1, \mathrm{t}-2) \\
\mathrm{x}_{4}(\mathrm{t}, \mathrm{t}-1, \mathrm{t}-2) ; \mathrm{x}_{5}(\mathrm{t}, \mathrm{t}-1, \mathrm{t}-2)\end{array}$ & - & $88.07 \%$ \\
\hline $\mathrm{y}_{8}$ & $\begin{array}{c}\mathrm{x}_{6}(\mathrm{t}, \mathrm{t}-1) ; \mathrm{x}_{6}(\mathrm{t}-2)^{2} \\
\sin \left(\mathrm{x}_{7}(\mathrm{t}-3)\right)^{2}\end{array}$ & $\begin{array}{c}\mathrm{y}_{8}(\mathrm{t}-1, \mathrm{t}-2, \mathrm{t}-3) ; \\
\mathrm{y}_{9}(\mathrm{t}-1, \mathrm{t}-2, \mathrm{t}-3) ; \\
\mathrm{y}_{10}(\mathrm{t}-1, \mathrm{t}-2, \mathrm{t}-3) ; \\
\mathrm{y}_{4}(\mathrm{t}, \mathrm{t}-1, \mathrm{t}-\mathrm{t}-2) ; \mathrm{x}_{6}(\mathrm{t}, \mathrm{t}-1, \mathrm{t}-2)\end{array}$ & $91.61 \%$ \\
\hline y9 & $\begin{array}{c}\cos \left(\mathrm{y}_{9}(\mathrm{t}-3)\right) ; \sin \left(\mathrm{y}_{9}(\mathrm{t}-3)\right)^{2} \\
\sin \left(\mathrm{x}_{6}(\mathrm{t})\right)^{2}\end{array}$ & $\begin{array}{c}\mathrm{y}_{8}(\mathrm{t}-1) ; \mathrm{y}_{9}(\mathrm{t}-1, \mathrm{t}-2) ; \\
\mathrm{y}_{10}(\mathrm{t}-1) ; \mathrm{y}_{4}(\mathrm{t}, \mathrm{t}-1, \mathrm{t}-2) \\
\mathrm{x}_{6}(\mathrm{t}, \mathrm{t}-1, \mathrm{t}-2) ; \mathrm{x}_{7}(\mathrm{t}, \mathrm{t}-1, \mathrm{t}-2)\end{array}$ & $92.68 \%$ \\
\hline$y_{10}$ & $\mathrm{y}_{4}(\mathrm{t})^{2 ;} \cos \left(\mathrm{y}_{9}(\mathrm{t}-3)\right)$ & $\begin{array}{c}\mathrm{y}_{8}(\mathrm{t}-1) ; \mathrm{y}_{8}(\mathrm{t}-2) ; \mathrm{y}_{9}(\mathrm{t}-1) ; \\
\mathrm{y}_{9}(\mathrm{t}-2), \mathrm{y}_{10}(\mathrm{t}-1) ; \mathrm{y}_{10}(\mathrm{t}-2) ; \mathrm{y}_{10}(\mathrm{t}- \\
3) ; \mathrm{y}_{4}(\mathrm{t}), \mathrm{y}_{4}(\mathrm{t}-1) ; \\
\mathrm{y}_{4}(\mathrm{t}-2) ; \mathrm{x}_{6}(\mathrm{t}) ; \mathrm{x}_{6}(\mathrm{t}-1), \mathrm{x}_{6}(\mathrm{t}-2) ; \\
\mathrm{x}_{7}(\mathrm{t}) ; \mathrm{x}_{7}(\mathrm{t}-1) ; \mathrm{x}_{7}(\mathrm{t}-2)\end{array}$ & $90.38 \%$ \\
\hline
\end{tabular}

Overall, the SCPC-CCS is represented by 1 ARX model and 2 NARX models. The pairwise correspondence between the 1-hour ahead prediction with the data from the high-fidelity model in Aspen ${ }^{\circledR}$ Dynamics is in the range $74 \%$ to $95 \%$. The lower 
correspondence is mainly for the variables in the SCPC part of the NARX model, which shows a highly nonlinear behavior and cross-interactions between the decision and dependent variables. The predictions of the reduced models are structurally similar to the behavior observed in the data from the high-fidelity model during PRBS step tests, as shown in Figures 4.2. to 4.4. Therefore, such models are considered suitable for the plantwide optimization framework. After the system identification was performed, the input and output variables data are normalized for better computational tractability of the gradientbased optimization.

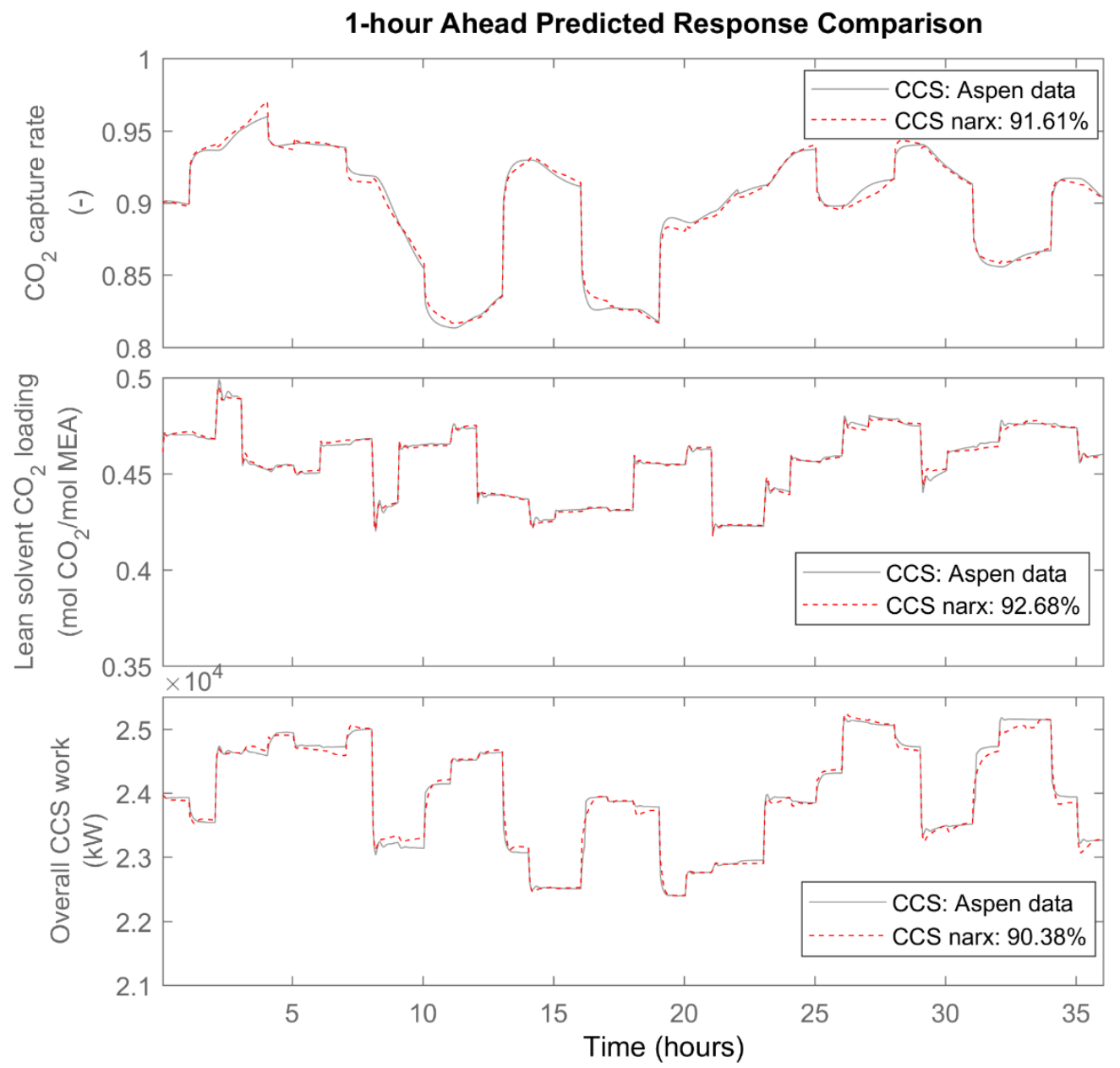

Figure 4.2. Comparison between 1-hour ahead model prediction with data from highfidelity Aspen model - CCS nonlinear ARX 


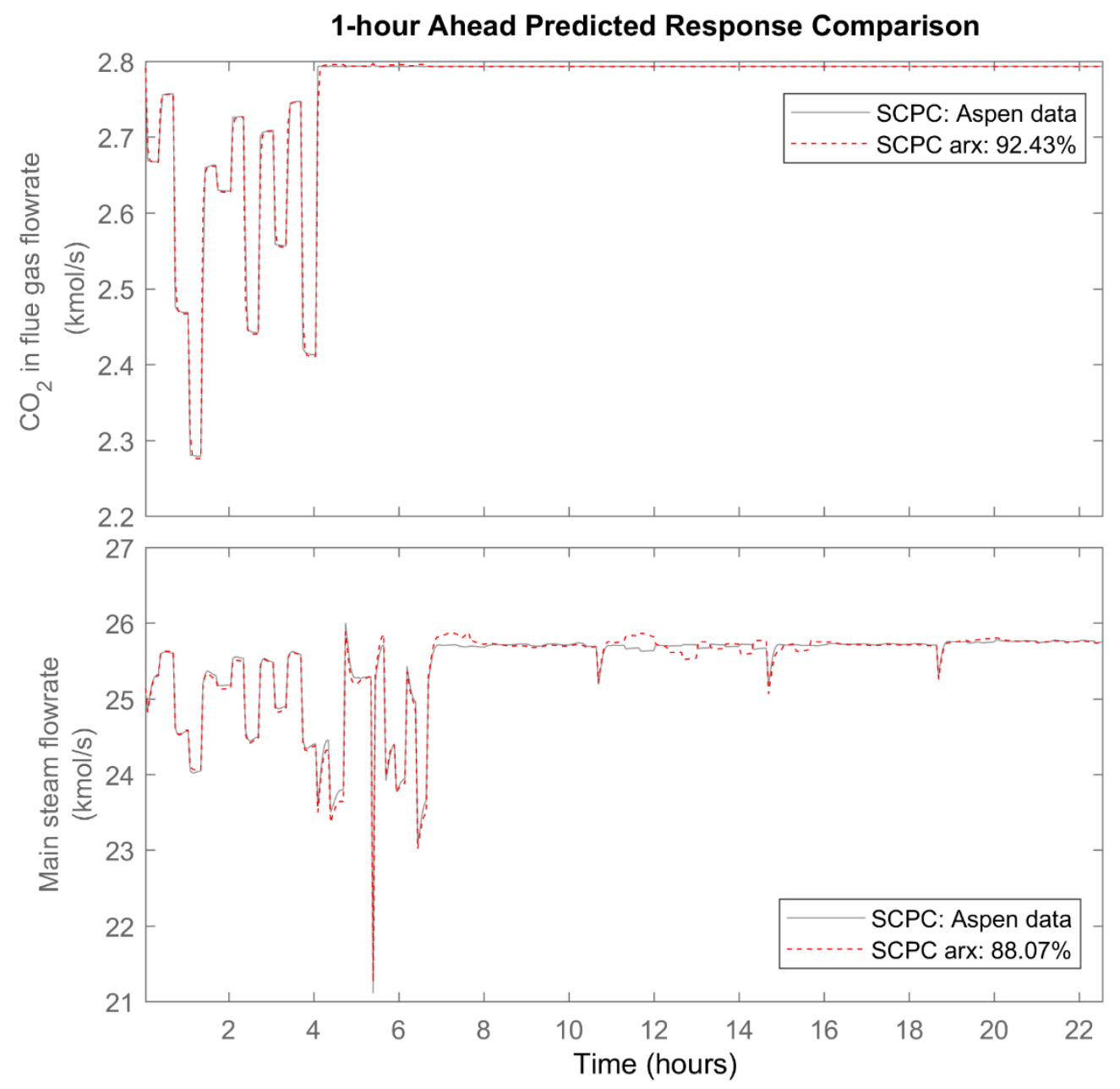

Figure 4.3. Comparison between 1-hour ahead model prediction with data from highfidelity Aspen model - SCPC linear ARX 


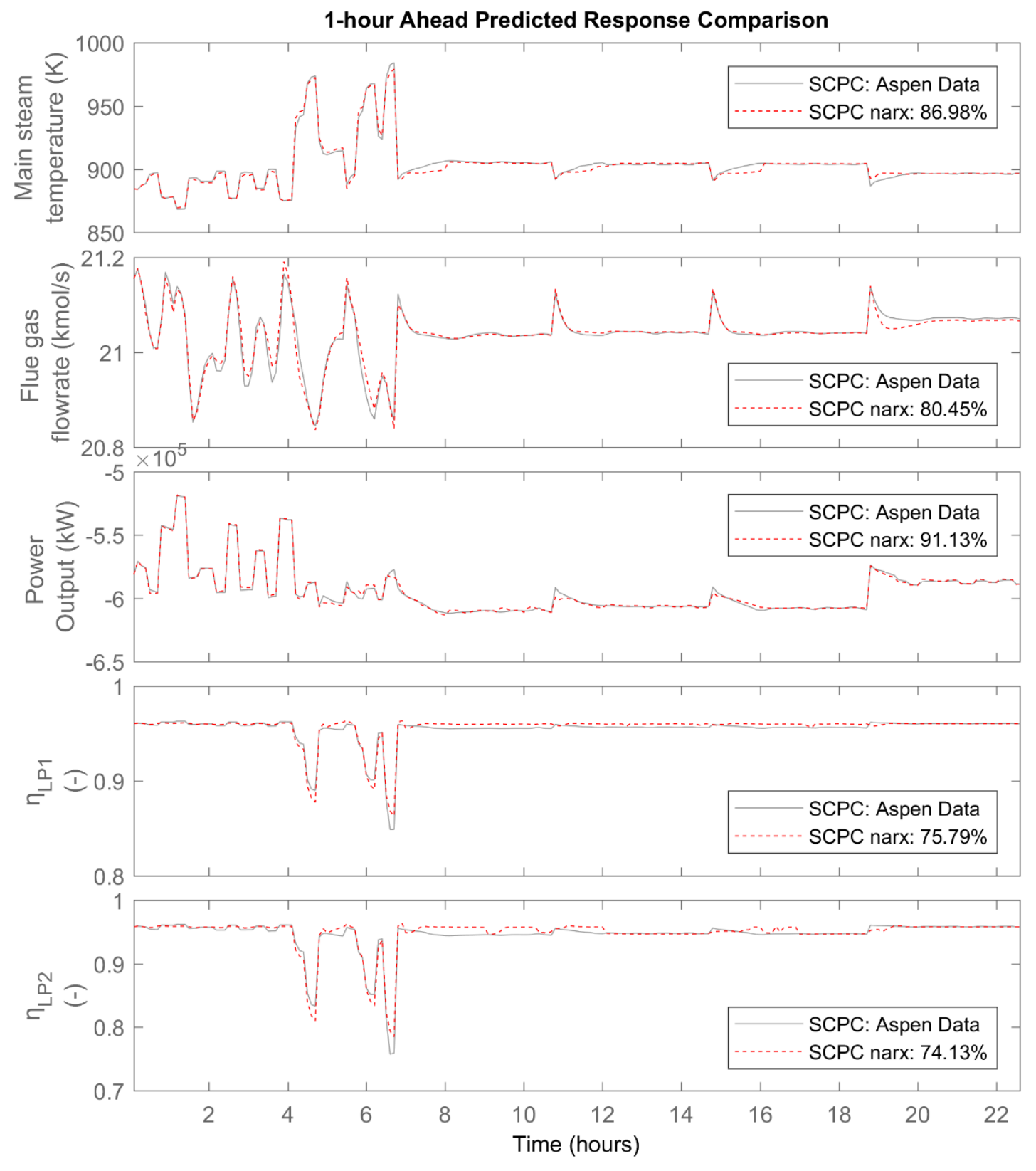

Figure 4.4. Comparison between 1-hour ahead model prediction with data from highfidelity Aspen model - SCPC nonlinear ARX

\subsection{Nonlinear Optimization of the SCPC-CCS}

\subsubsection{Problem Statement}

The objectives of the NMPO framework are economic (ECON) and environmental (ENV). The economic objective aims to minimize overall power plant operating cost while the environmental objective aims to maximize the amount of carbon being captured. 
Equations (4.9) and (4.10) show the definition of the objectives. The optimization problem has additional system constraints per time step (4), shown in Equations (4.11) to (4.14). Including the slack variables of the 3 inequality constraints in Equations (4.12) to (4.14), the optimization problem has 10 decision variables per time step. For instance, for an optimization horizon of $t_{2}$ and time step of $\Delta \mathrm{t}$, there are $10 t_{2} \Delta \mathrm{t}^{-1}$ decision variables, $4 t_{2} \Delta \mathrm{t}^{-1}$ constraints, and the first order-derivatives have the dimensions

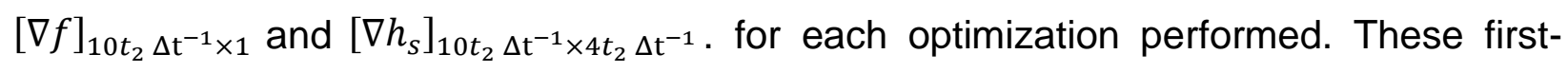
order derivatives need to be obtained and all optimizations solved within the implementation time horizon. The search space for the optimal solution is considered the same as the normalized input ranges for the system identification, unless otherwise specified.

$$
\begin{gathered}
\min \operatorname{ECON}(\varphi)=\Delta t \sum_{t}\left(\begin{array}{c}
\text { Coal price }- \text { Electricity } \\
+ \text { Transportation }+ \text { Storage } \\
-E O R-\text { Carbon credit }+ \text { Carbon tax }
\end{array}\right) \\
\max \operatorname{ENV}(\varphi)=\Delta t \sum_{t}\left(\mathrm{CO}_{2}\right. \text { in flue gas flowrate } \\
\left.\times \mathrm{CO}_{2} \text { capture rate }\right) \\
\text { Power output }=\text { Demand } \\
\eta_{\mathrm{LP} 1} \geq \eta_{\min } \\
\eta_{\mathrm{LP} 2} \geq \eta_{\min }
\end{gathered}
$$

in which $\Delta t$ is the discretization time step of $0.1 \mathrm{~h}$. Regarding the economic objective, the Coal price is the price of the coal (US\$/h), Electricity represent the wholesale of the electricity (US\$/h), Transportation is the cost related to $\mathrm{CO}_{2}$ transportation, such as onshore/off-shore and length of pipelines, and Storage is the cost related to $\mathrm{CO}_{2}$ storage, such as onshore/off-shore and reuse of wells. Moreover, for carbon policies, EOR is the credit due to $\mathrm{CO}_{2}$ utilization for enhanced oil recovery, Carbon credit is the value for the carbon captured in a cap\&trade scenario, and Carbon tax is the tax embedded in the carbon released into the atmosphere. Regarding the environmental objective, Carbon Captured is the amount of $\mathrm{CO}_{2}$ captured. 
As this formulation includes costs from different years, all cost values are scaled considering the 2019 Chemical Engineering Plant Cost Index (CEPCI) (CEP, 2021) and normalized to the same basis chosen as year 2000, as shown in Equation (4.15).

$$
\operatorname{Cost}_{2019}=\text { Cost }_{\text {ref }} \times \frac{\text { CEPCI }_{2019}}{\text { CEPCI }_{\text {ref }}}
$$

Specifically, the terms in the objective function are further defined in Equations (4.16) to (4.22):

$$
\begin{gathered}
\text { Coal price }=x_{1} \times \text { price }_{\text {coal }} \\
\text { Electricity }=y_{1} * \text { overall price }_{\text {electricity }} \\
\text { Transportation }=y_{4} \times y_{8} \times h_{2} \\
\text { Storage }=y_{4} \times y_{8} \times h_{3} \\
\text { EOR }=h_{5} \times y_{4} \times y_{8} \\
\text { Carbon Credit }=\text { CCmarket } \times y_{4} \times y_{8} \\
\text { Carbon tax }=h_{4, T A X} \times y_{4}^{2}\left(1-y_{8}\right)
\end{gathered}
$$

in which $h_{2}$ is the recommended cost for onshore $\mathrm{CO}_{2}$ transportation pipelines (US\$/ton $\mathrm{CO}_{2} / 250 \mathrm{~km}$ ); $h_{3}$ is the recommended cost for onshore $\mathrm{CO}_{2}$ storage (US $\$$ /ton $\mathrm{CO}_{2}$ ); CCmarket is the hypothetical change in the market price value of the $\mathrm{CO}_{2}$ credit according to the amount of carbon captured (US\$/ton $\left.\mathrm{CO}_{2}\right) ; h_{4, T A X}$ is the tax value imposed to the amount of carbon released (US\$/ton $\mathrm{CO}_{2}$ ) based on existing similar policies (CTC, 2021); and $h_{5}$ is the recommended value for enhanced oil recovery according to the amount of carbon captured (US\$/ton $\mathrm{CO}_{2}$ ). Recommended values for the costs/credits associated with Transportation, Storage and EOR are obtained from the literature (Rubin et al., 2015).

The forcing function profiles of the carbon credit, electricity price (PJM, 2019), and electricity demand (PJM, 2019) used in this work are shown in Figure 4.5. The price of coal from Illinois Basin considered is in average $35.30 \$$ /ton (EIA, 2021). If the $\mathrm{CO}_{2}$ is sold as EOR, there is no cost regarding carbon storage. Also, the threshold values in Equations (4.12) to (4.14) are $T_{\max }=915 \mathrm{~K}$ and $\eta_{\min }=70 \%$. 


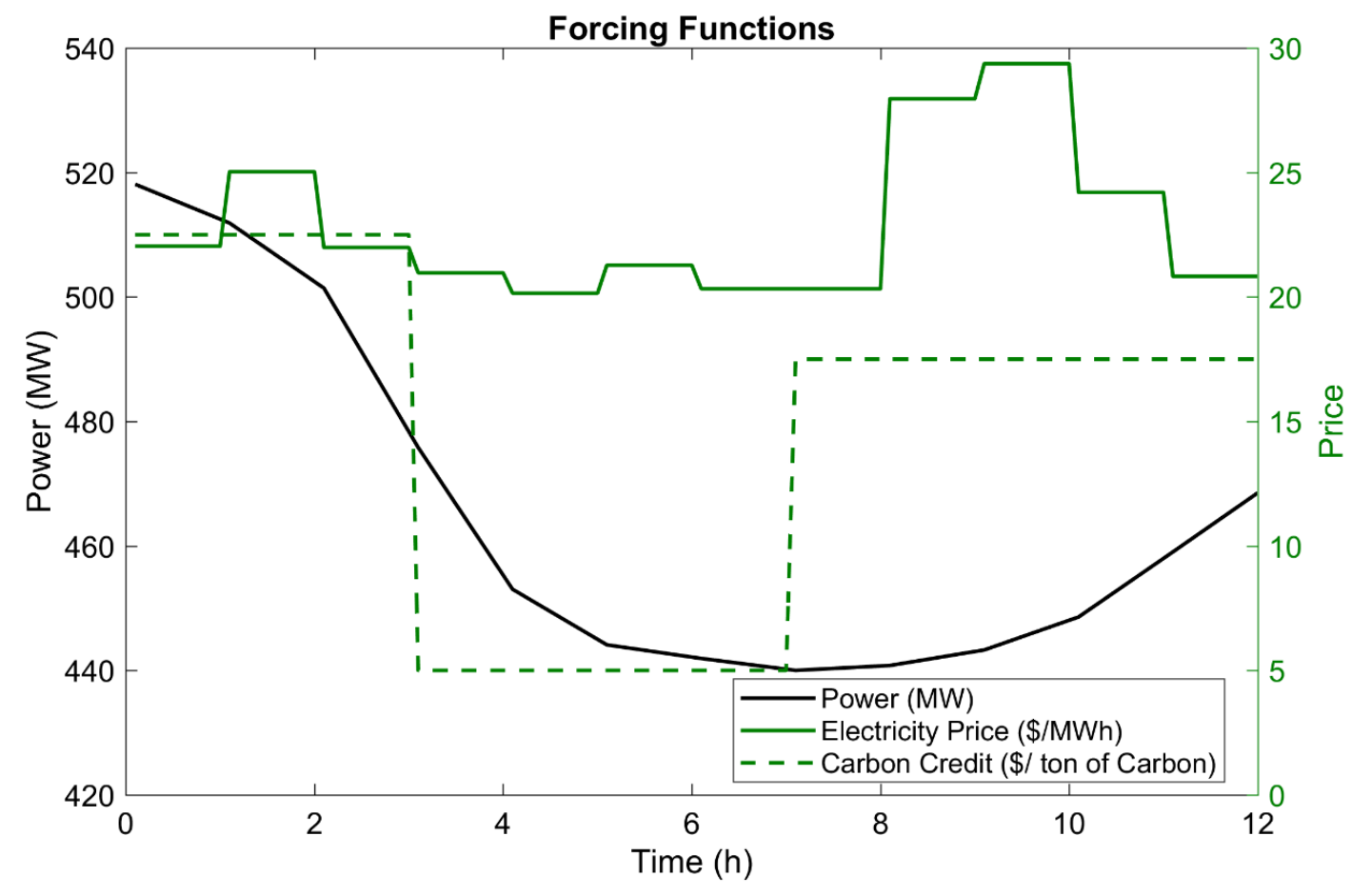

Figure 4.5. Power demand cycling profile (left axis), electricity price and carbon credit (right axis) for 12-hour horizon

The ParPSO parameters are shown in Table 4.3. Considering these values, a reasonable feasible guess is always found at the end of the maximum number of iterations.

Table 4.3. ParPSO parameters for the NMPO framework

\begin{tabular}{|c|c|c|}
\hline Parameters & Economic Objective & Environmental Objective \\
\hline$\Phi_{1}$ & 2.1 & 2.3 \\
\hline$\Phi_{2}$ & 2.2 & 2.3 \\
\hline$\gamma$ & 0.97 & 0.96 \\
\hline Particle in swarm & 18 & 12 \\
\hline Maximum iterations & 10 & 8 \\
\hline Parallel workers & 8 & 8 \\
\hline
\end{tabular}


Regarding the constraints in the PSO formulation, the equality constraint defined in Equation (4.11) is not enforced as the initial guess for the IPOPT only requires feasibility with respect to the inequality constraints. The inequality constraint violation was enforced by using a static penalty function, which heavily hinders the objective function if the constraints are violated and steer the particles away from unfavorable regions.

Also, considering that the electricity price predictions tend to be less accurate farther they are from the current time, an opportunity variable $\left(w_{o p}\right)$ was implemented as the cost of opportunity. A similar strategy has already been adopted in literature (Bankole et al., 2018) to somehow account for uncertainty in long time horizons without increasing the computational time. The cost of opportunity is considered as the cost of forfeiting a "rightnow" gain to achieve an overall higher gain due to potential future conditions; however, as the time horizon moves, the costs may also change considerably, and the future scenario may never occur. Therefore, the role of this new variable is to introduce a bias in the algorithm such that it will slightly favor the earlier (or more accurate) electricity prices when compared to later electricity prices within an optimization horizon, as shown in Equation (4.23):

$$
\text { Overall price }_{\text {electricity }}=w_{o p} * \text { price }_{\text {electricity }}
$$

in which, $w_{o p}$ is a weight vector in which each element has maximum value of 1 and price is the vector with the electricity prices $(\$ / \mathrm{kWh})$. In this case, the $w_{o p}=\left[\begin{array}{ll}1.00 & 0.950 .90\end{array}\right]$, as the maximum simulated optimization horizon is $3 \mathrm{~h}$.

The implementation horizon is $1 \mathrm{~h}$ based on the time scale that the forcing functions usually change. In the current application, the forcing functions are likely to change in an hourly manner, as shown in Figure 4.5. With the implementation horizon being $1 \mathrm{~h}$, the NMPO can operate with the most updated forecasted values for the forcing functions. Therefore, the real-time constraint to obtain the optimal trajectories is $1 \mathrm{~h}$.

To determine the optimization horizon, three horizons of $1 \mathrm{~h}, 2 \mathrm{~h}$, and $3 \mathrm{~h}$ are tested using the modified Tchebycheff weighted metric method for $\mathrm{T}=4 \mathrm{~h}$ simulated horizon, considering equal weights to each objective $\left(w_{1}=w_{2}=0.5\right)$. As a remark, the simulation with optimization horizon of $1 \mathrm{~h}$ still calculates the optimal trajectory for that time interval but without any prediction capability, as the optimization and the implementation horizons are 
the same. Considering the minimization formulation, results in Figure 4.6 show the benefit of having an optimization horizon longer than the implementation horizon. In particular, a prediction capability allowed the algorithm to set up optimal conditions ahead of time to reach lower costs while maintaining the environmental performance.

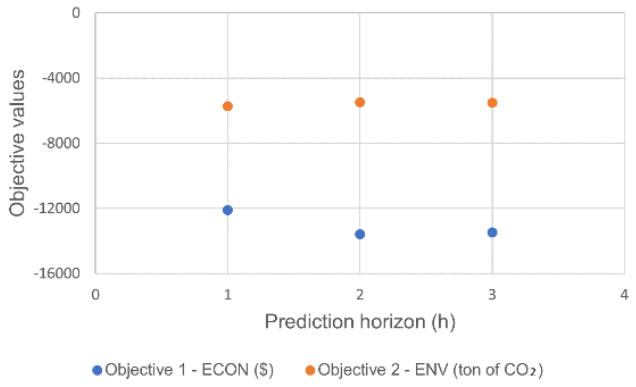

Figure 4.6. Objective values for implementations with different optimization horizons

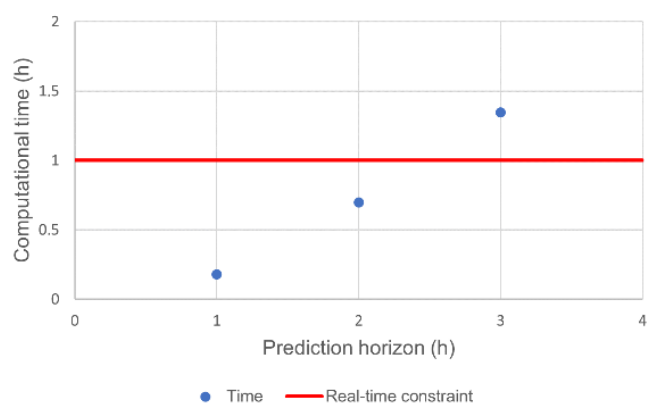

Figure 4.7. Computational times for implementations with different optimization horizons (dots) and the considered realtime constraint (line)

Figure 4.7 presents the average computational times to calculate the four piecewise implementation horizon and respective optimization horizons of $1 \mathrm{~h}, 2 \mathrm{~h}$, and $3 \mathrm{~h}$. The result shows that the longer is the optimization horizon, the longer is the computational time. At times (e.g., for an optimization horizon of $3 \mathrm{~h}$ ), the real-time constraint implementation is violated without significant gain in the objectives' values.

This exploratory assessment demonstrated that the most suitable optimization and implementation horizons while applying this framework depends on the problem forcing functions time scale and the energy systems models. Considering the current application and results, the chosen optimization horizon is of $2 \mathrm{~h}$.

For an optimization horizon of $2 \mathrm{~h}$ and time step of $0.1 \mathrm{~h}$, there are 200 decision variables, 80 constraints, and the first order-derivatives have the dimensions $[\nabla f]_{200 \times 1}$ and $\left[\nabla h_{s}\right]_{200 \times 80}$. for each optimization performed. These first-order derivatives need to be obtained and all optimizations solved within the implementation time horizon of $1 \mathrm{~h}$. The NMPO method performs up to 5 per optimization implementation horizon of $1 \mathrm{~h}$. 
After the parameters are set, four case studies are performed using the different multiobjective methods, modified Tchebycheff and the $\epsilon$-constraint method.

\subsubsection{Optimization Results}

Prior to the full implementation, a study is performed for the Tchebycheff MOO component when selecting the optimal compromise considering different weight vectors as a proof of concept to assess the distribution of compromises. This study is performed in the carbon capture subsystem and it is considered that the flue gas flowrate is proportional to the power demand, shown in Figure 4.5. The optimal compromises chosen by the MOO component are plotted against a previously mapped objective space. Afterwards, four case studies are presented regarding the full SCPC-CCS system. The scenarios analyzed correspond to the optimal trajectories up to $\mathrm{T}=12 \mathrm{~h}$ with optimization horizon of $\mathrm{t}_{2}=2 \mathrm{~h}$, and implementation horizon of $\mathrm{t}_{1}=1 \mathrm{~h}$, considering different carbon capture policies and different MOO methods, namely the modified Tchebycheff and the $\epsilon$-constraint method.

Regarding the preliminary study, the entire feasible space is first mapped using exhaustive search to delineate the Pareto Front (shown in blue in Figure 4.8). Then, weight values are uniformly changed and attributed in the modified Tchebycheff-based MOO formulation to assess the effect of this selection on the optimal distribution of compromises. The optimal compromises for the different weights (shown as red dots in Figure 4.8) are plotted and superimposed to the previously mapped feasible space. All values are scaled considering their respective positions to the offline mapping, which is also scaled using $w_{1}=w_{2}=0.5$.

From the analysis of Figure 4.8, the optimal compromises are relatively well distributed along the Pareto front with no signs of clustering for different weight vectors, although an increase of sensitivity can be detected closer to the extremity of $w_{1}=1$. Under this method, there is no need to compute the entire Pareto front while still selecting a Pareto-optimal compromise. However, caution should be used if the weight vector is disproportional, such as $\mathrm{w}_{1}=0.1$ and $\mathrm{w}_{2}=0.9$. 


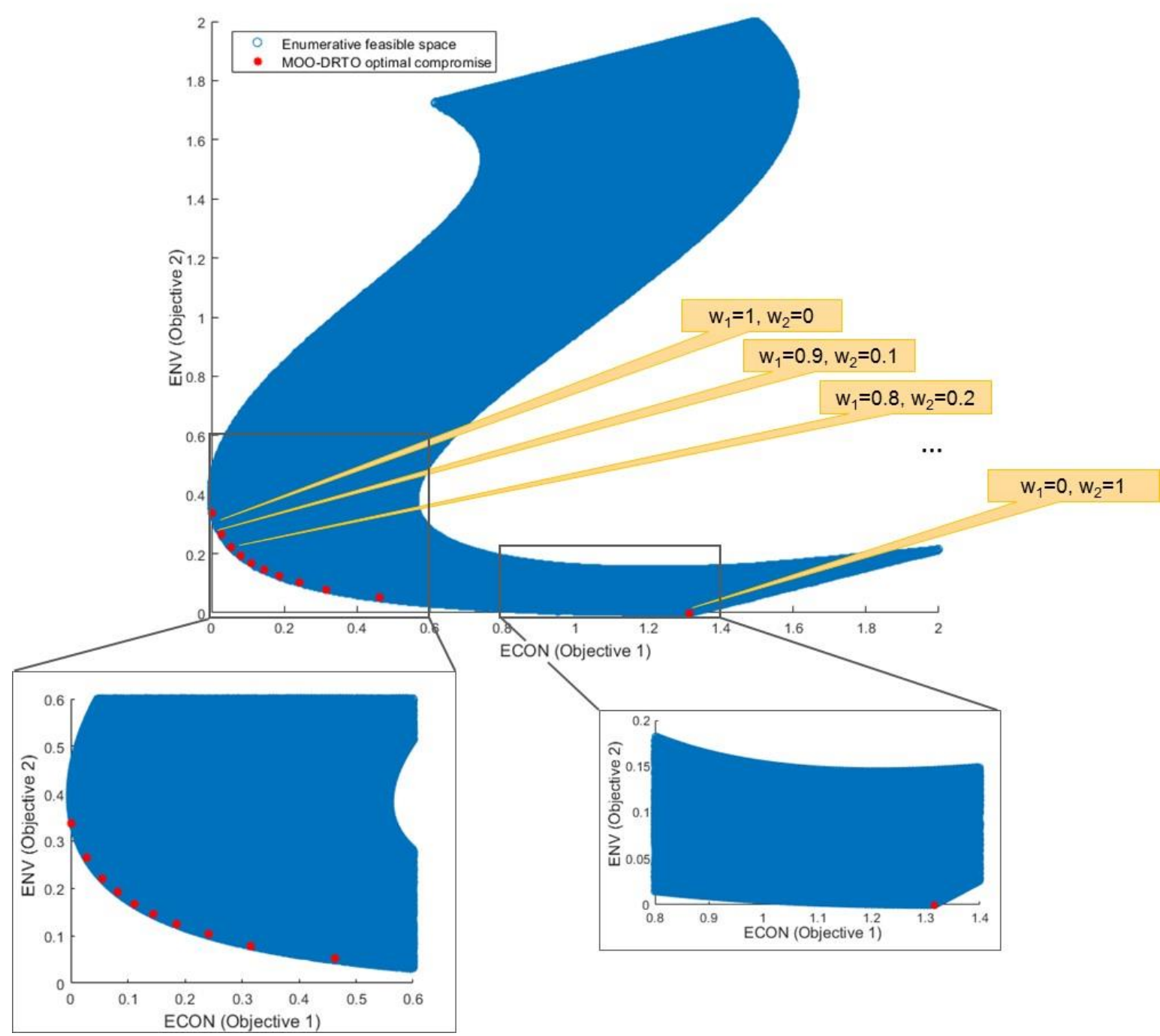

Figure 4.8. Feasible space and distribution of optimal compromise and the optimal compromise according to the weight vector along the Pareto front

Regarding the case studies, two carbon policy scenarios are simulated in both $\mathrm{MOO}$ methods. The CEPCI adjusted values of the parameters adopted are shown in Table 4.4. Case 1 represents a scenario with absence of market for the captured $\mathrm{CO}_{2}$ as EOR and absence of carbon tax policy. Case 2 represents a scenario with greater incentives for capturing carbon with available market for $\mathrm{CO}_{2}$ as $\mathrm{EOR}$ and high taxation on carbon, which is within the range of current policies such as the FUTURE Act Policy 45Q (FUTURE Act, 2017). The weights adopted for the modified Tchebycheff and the $\epsilon$-constraint formulation are $w_{1}=w_{2}=w_{2}^{\epsilon \text {-const }}=0.5$. Specifically, the ECON objective 
is chosen as the MOO objective and the ENV objective is reformulated as a constraint for the $\epsilon$-constraint method.

Table 4.4. Optimization parameters for carbon policy scenarios - Cases 1 and 2

\begin{tabular}{|c|c|c|c|}
\hline Parameters & Unit & Case 1 & Case 2 \\
\hline$h_{2}$ & $\left(2019 \mathrm{US} \$ /\right.$ ton $\left.\mathrm{CO}_{2}\right)$ & 5.2472 & 1.8205 \\
\hline$h_{3}$ & $\left(2019 \mathrm{US} \$ /\right.$ ton $\left.\mathrm{CO}_{2}\right)$ & 13.9212 & 7.4960 \\
\hline$h_{4, T A X}$ & $\left(2019 \mathrm{US} \$ /\right.$ ton $\left.\mathrm{CO}_{2}\right)$ & 0 & 32.1259 \\
\hline$h_{5}$ & $\left(2019 \mathrm{US} \$ /\right.$ ton $\left.\mathrm{CO}_{2}\right)$ & 0 & 42.8345 \\
\hline
\end{tabular}

Figure 4.9 shows that the power output constraint is satisfied at all times for Cases 1 and 2 during the optimizations considering the economic objective, environmental objective, and the multi-objective problem formulated using the modified Tchebycheff and the $\epsilon$-constraint methods.

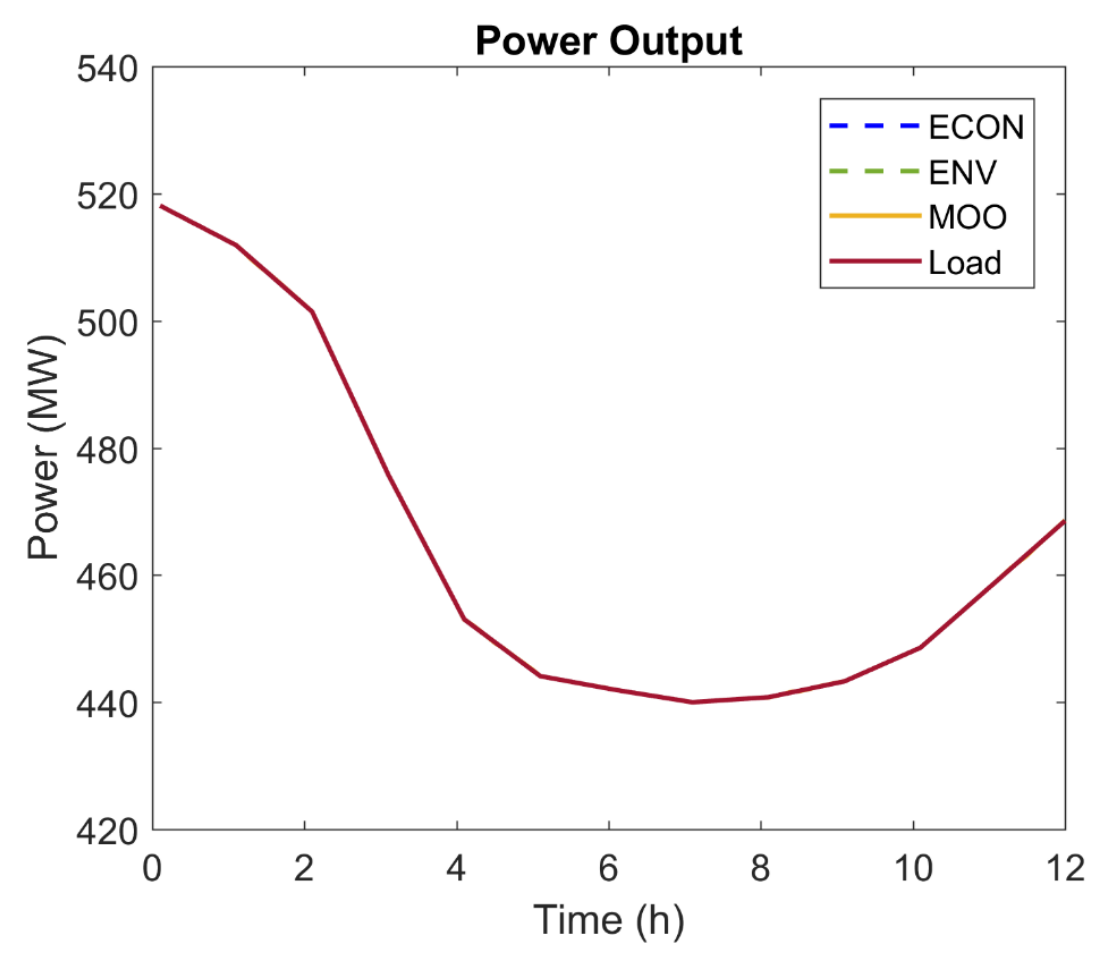

Figure 4.9. Satisfied SCPC power output constraint 
Figures 4.10 to 4.17 show the objective values for the optimization and implementation horizons of the economic, environmental, and the modified Tchebycheff-based NMPO for Cases 1 and 2. In Figures 4.10 and 4.11 for Case 1 and Figures 4.14 and 4.15 for Case 2 , the economic optimization always obtains the lowest objective value while the environmental optimization operates under higher costs, mostly due to the operation of CCS. The MTch MOO objective values remain balanced between both objectives. Corresponding behavior is observed in Figures 4.12 and 4.13 for Case 1 and Figures 4.16 and 4.17 for Case 2, in which the environmental optimization always obtains the highest value for carbon captured and the economic optimization not necessarily attempt to maximize the carbon captured, with MTch MOO again remaining in between both objectives. Furthermore, the results presented in Figures 4.10 to 4.17 show that the objective values between the optimization and implementation horizon are proportional to each other. The generated trajectories by the MTch MOO do not present a "bang-bang" behavior and remain balanced between the economic and environmental objectives. The "bang-bang" effect is considered in this dissertation as the effect of the trajectories going from one extreme to the other in a cyclic manner and in a short amount of time.

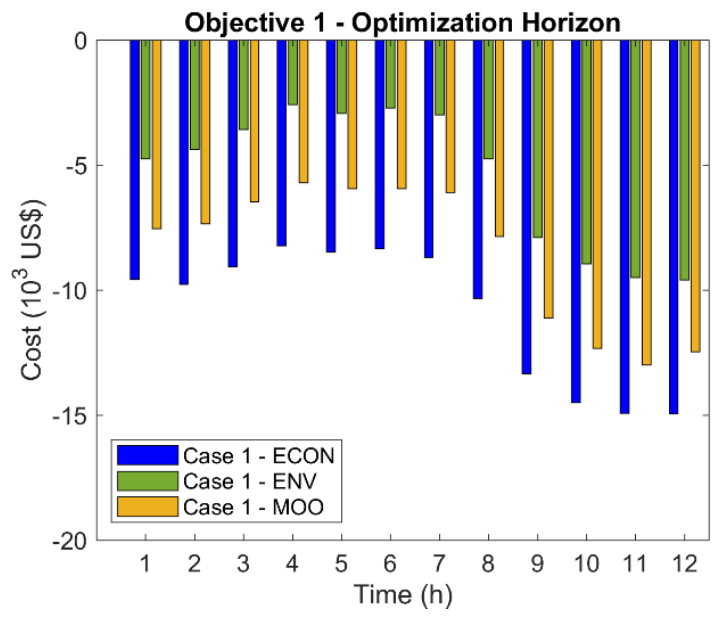

Figure 4.10. Economic objective value for optimization horizon of the economic, environmental, and MTch multi-objective optimizations - Case 1

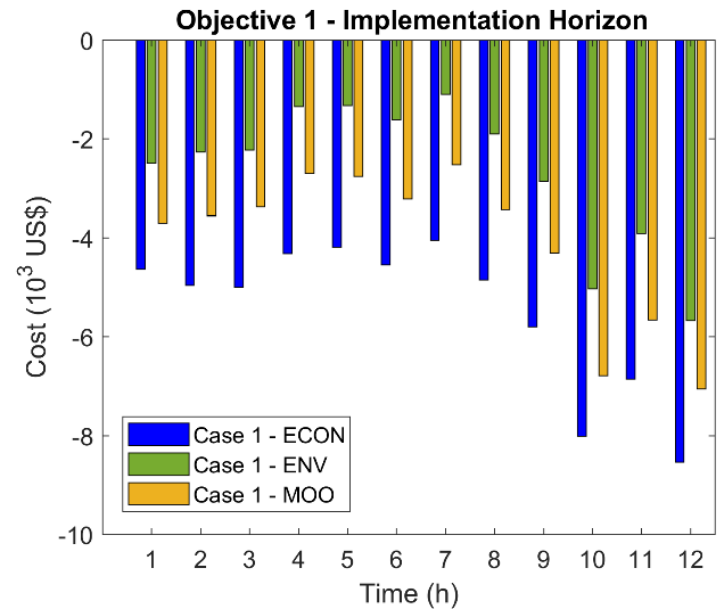

Figure 4.11. Economic objective value for implemented horizon of the economic, environmental, and MTch multi-objective optimizations - Case 1 


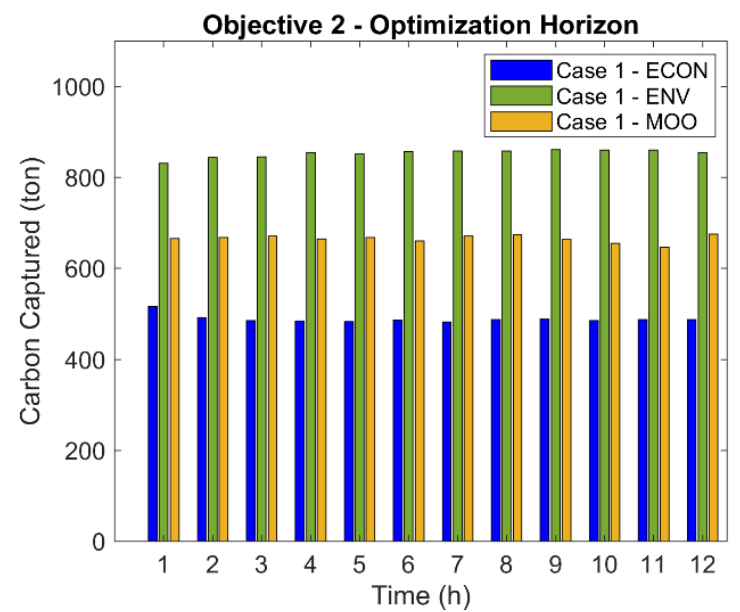

Figure 4.12. Environmental objective value for optimization horizon of the economic, environmental, and MTch multi-objective optimizations - Case 1

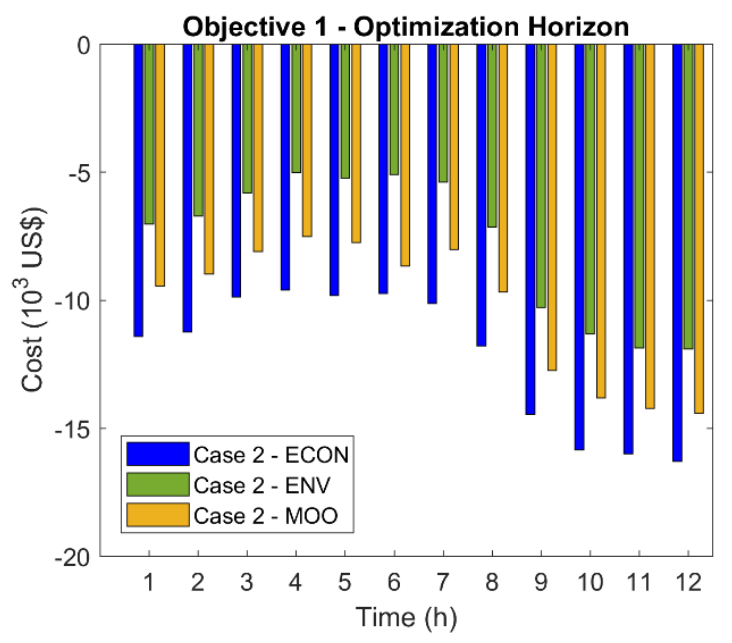

Figure 4.14. Economic objective value for optimization horizon of the economic, environmental, and MTch multi-objective optimizations - Case 2

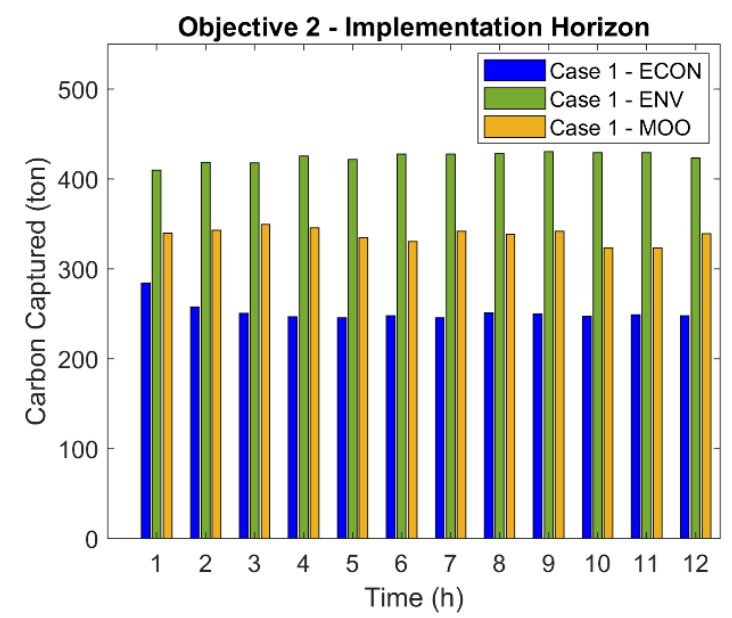

Figure 4.13. Environmental objective value for implemented horizon of the economic, environmental, and MTch multi-objective optimizations - Case 1

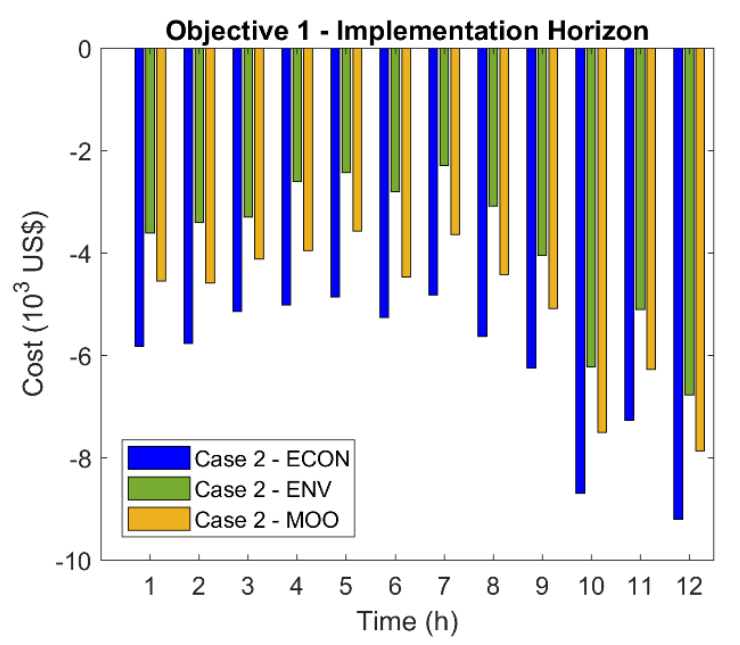

Figure 4.15. Economic objective value for implemented horizon of the economic, environmental, and MTch multi-objective optimizations - Case 2 


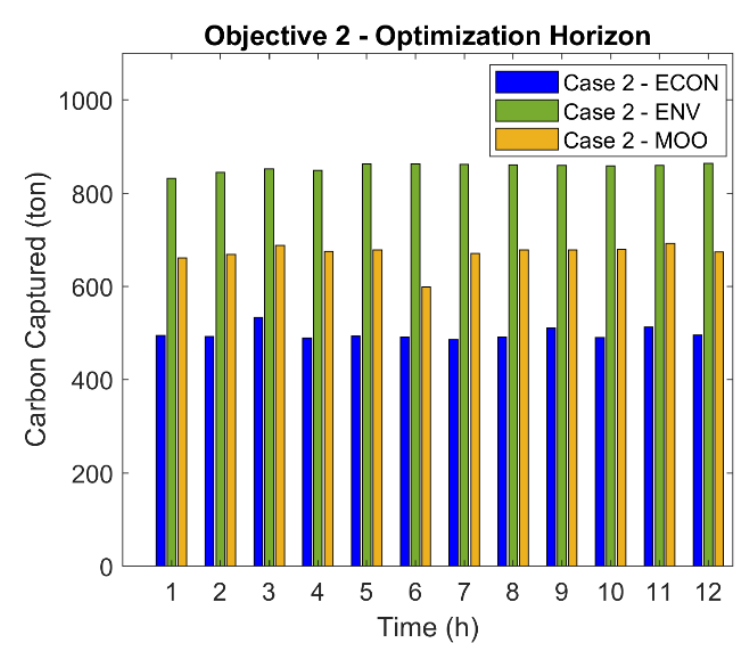

Figure 4.16. Environmental objective value for optimization horizon of the economic, environmental, and MTch multi-objective optimizations - Case 2

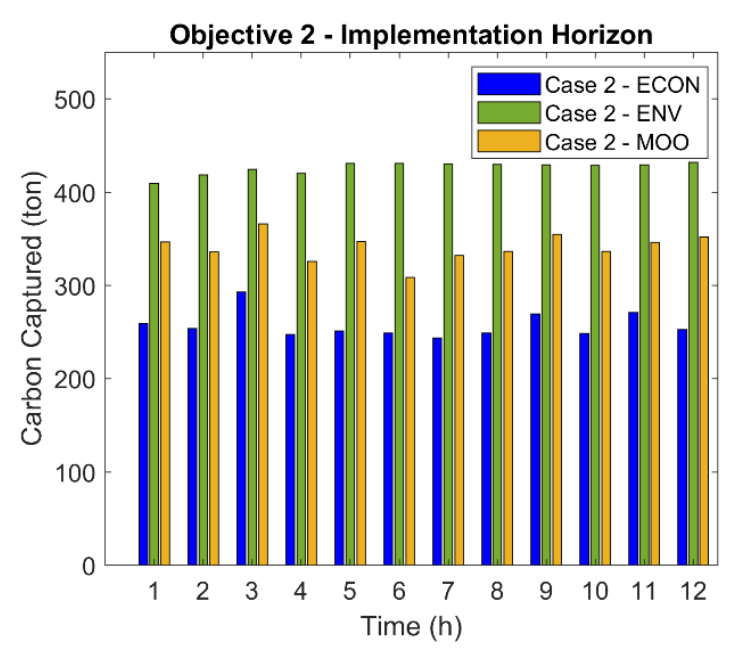

Figure 4.17. Environmental objective value for implemented horizon of the economic, environmental, and MTch multi-objective optimizations - Case 2

Considering the objective values of Cases 1 and 2, the economic carbon incentives do not significantly affect the standalone environmental optimization as the ENV objective do not account for any economic parameter in the objective. Such incentives, however, affect the economic objective standalone optimization and, consequently, the MOO optimization result. For the 12-hour horizon of Case 2, there was an increase of approximately 65 ton of $\mathrm{CO}_{2}$ captured for the economic optimization and 63 ton of $\mathrm{CO}_{2}$ captured for the MOO compromise when compared to Case 1.

In general, the economic optimization operates the power plant at lower auxiliary power and reduces the amount of low-pressure steam extracted from the turbines for solvent recovery. It also operates at lower main steam pressure and higher main steam temperature when compared to the environmental optimization, as depicted in Figures 4.18 to 4.21 . The results obtained using the NMPO framework are similar to a sliding pressure strategy, which relies on the principle that a rotating turbine requires less pressure as load is reduced and vice-versa. A fast-response load reserve can be accessed by opening a throttle valve or an admission valve. 
However, most baseload power plants being cycled under VRE are existing power plants that are not designed to be operated in varying temperature and pressure. For instance, recommended modifications to change from constant to sliding operating mode include, but are not limited to, furnace resizing, equipment reinforcements, construction material, inclusion of new throttle and admission valves. Therefore, considering that the baseload SCPC-CCS is designed to operate at constant pressure and temperature, an input constraint on the main steam pressure is considered in this study, by limiting the value of the main steam pressure to be higher than 25.4 MPa and lower than 26.4 MPa. This analysis shows that, although the compromise between objectives is automatically selected by the NMPO framework, chemical engineering concepts and specific power plant operations knowledge are key to properly employ the developed framework. Moreover, the main steam temperature is always above $840 \mathrm{~K}$, which could be a limiting value depending on the age and construction material of the pipelines. Thus, the varying operating temperature and pressure motivate the implementation in the future of an equipment stress-based constraint, similar to the constraints included in the dynamic dispatch approach detailed in Chapter 5.

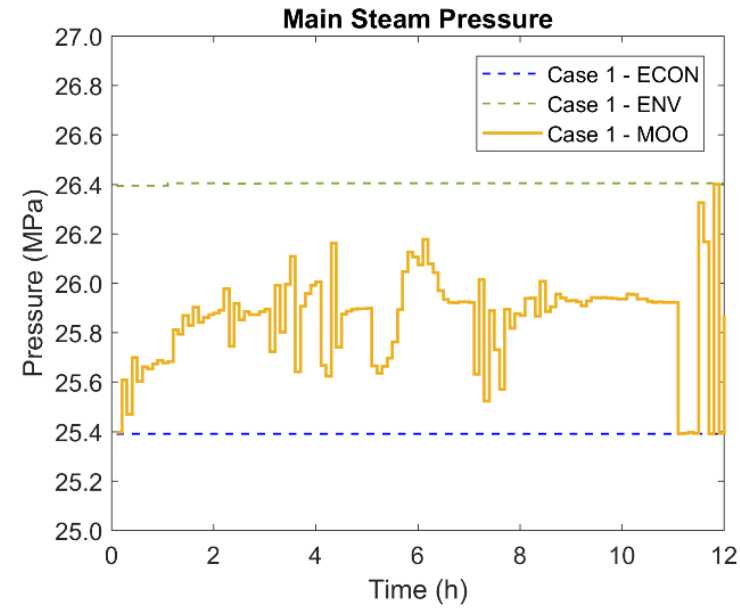

Figure 4.18. Main steam operating pressure for economic, environmental and MTch multi-objective optimizations -

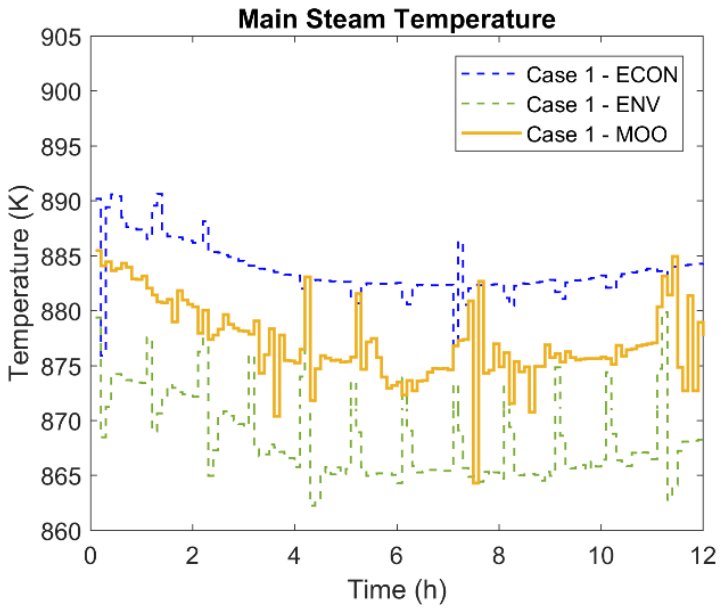

Figure 4.19. Main steam operating temperature for economic, environmental and MTch multi-objective optimizations -

Case 1 


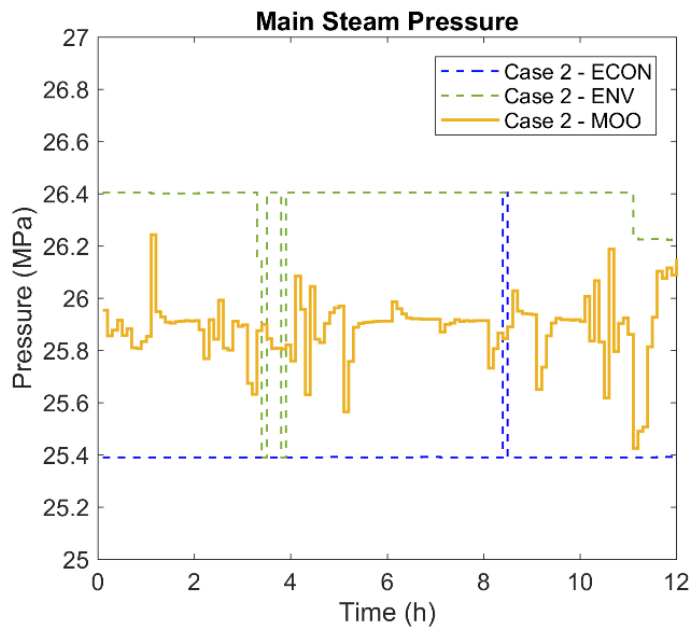

Figure 4.20. Main steam operating pressure for economic, environmental and MTch multi-objective optimizations -

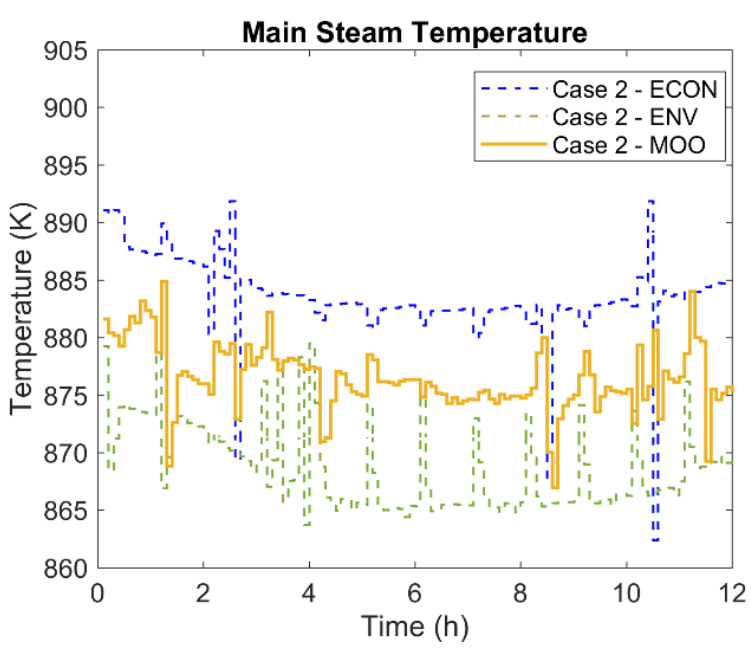

Figure 4.21. Main steam operating temperature for economic, environmental and MTch multi-objective optimizations -

Case 2

The most relevant MTch MOO input and output trajectories for Cases 1 and 2 are shown in Figures 4.22 to 4.30. The outputs are the main steam flowrate, carbon captured, lean solvent $\mathrm{CO}_{2}$ loading, and the low-pressure steam extracted for solvent recovery.
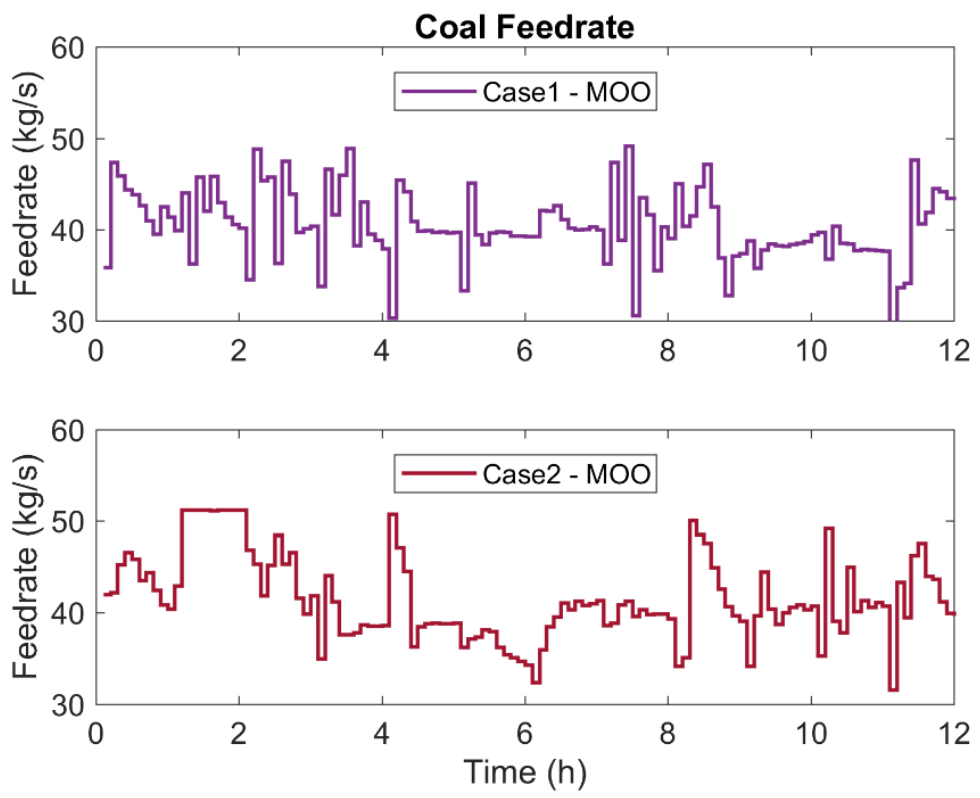

Figure 4.22. Coal feedrate for Cases 1 and 2 obtained with MTch multi-objective optimization 

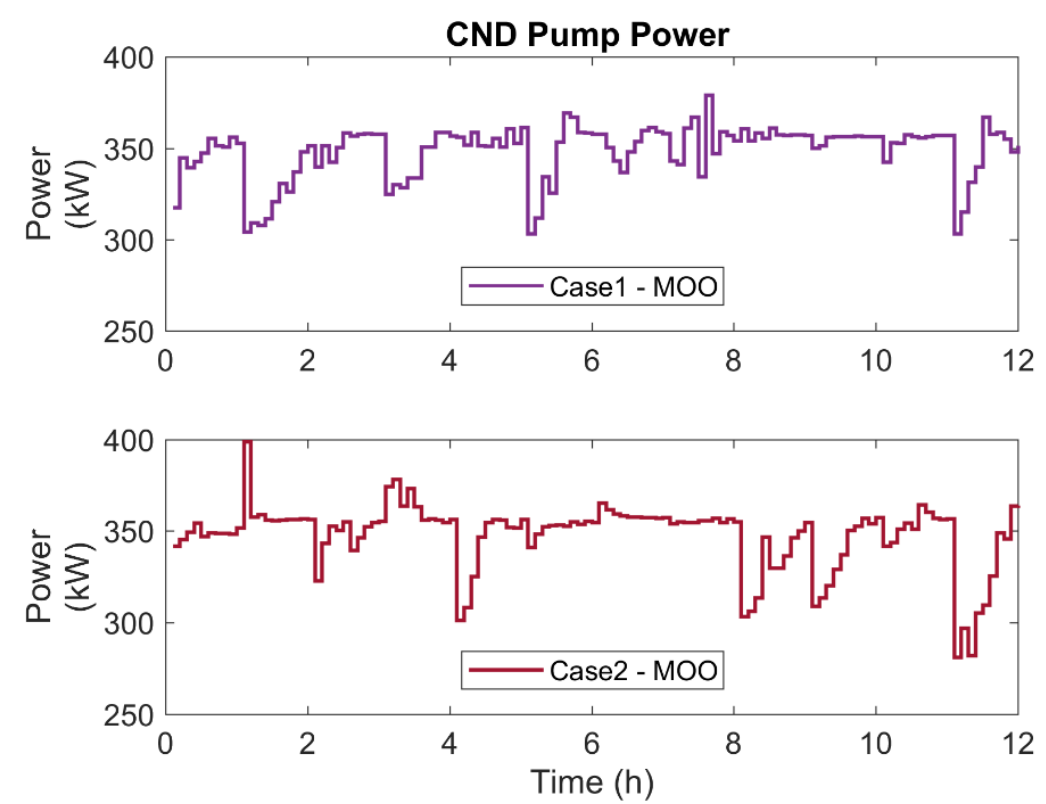

Figure 4.23. CND pump power for Cases 1 and 2 obtained with MTch multi-objective optimization
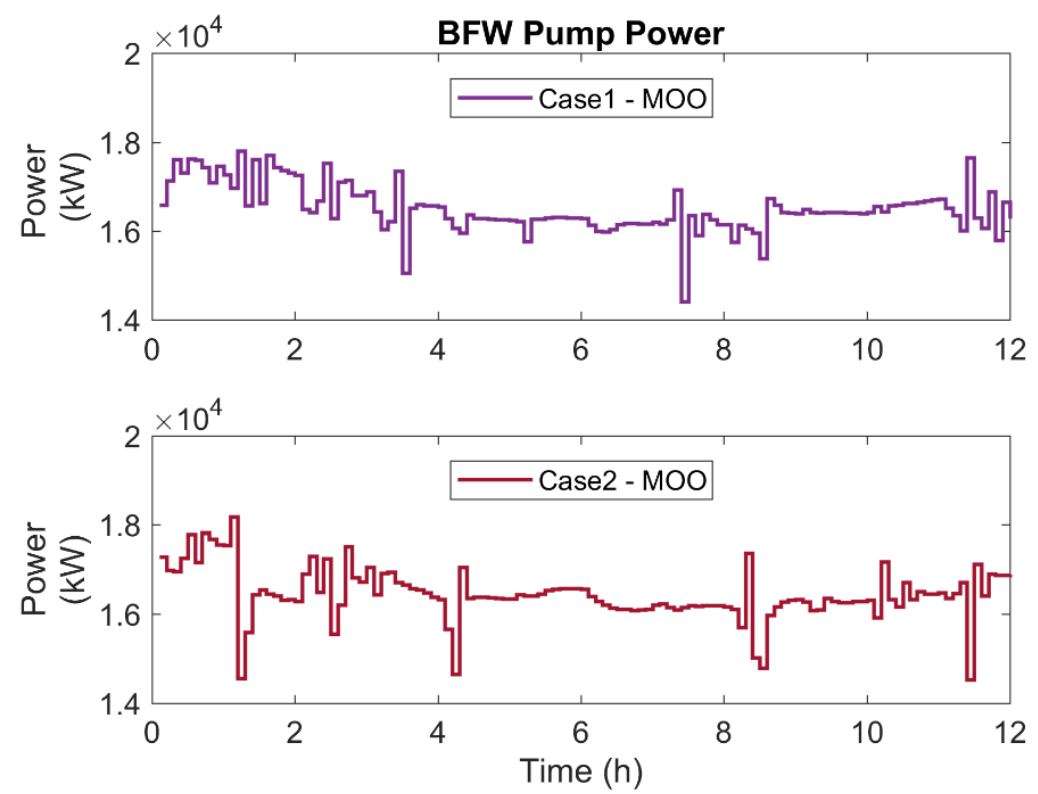

Figure 4.24. BFW pump power for Cases 1 and 2 obtained with MTch multi-objective optimization 

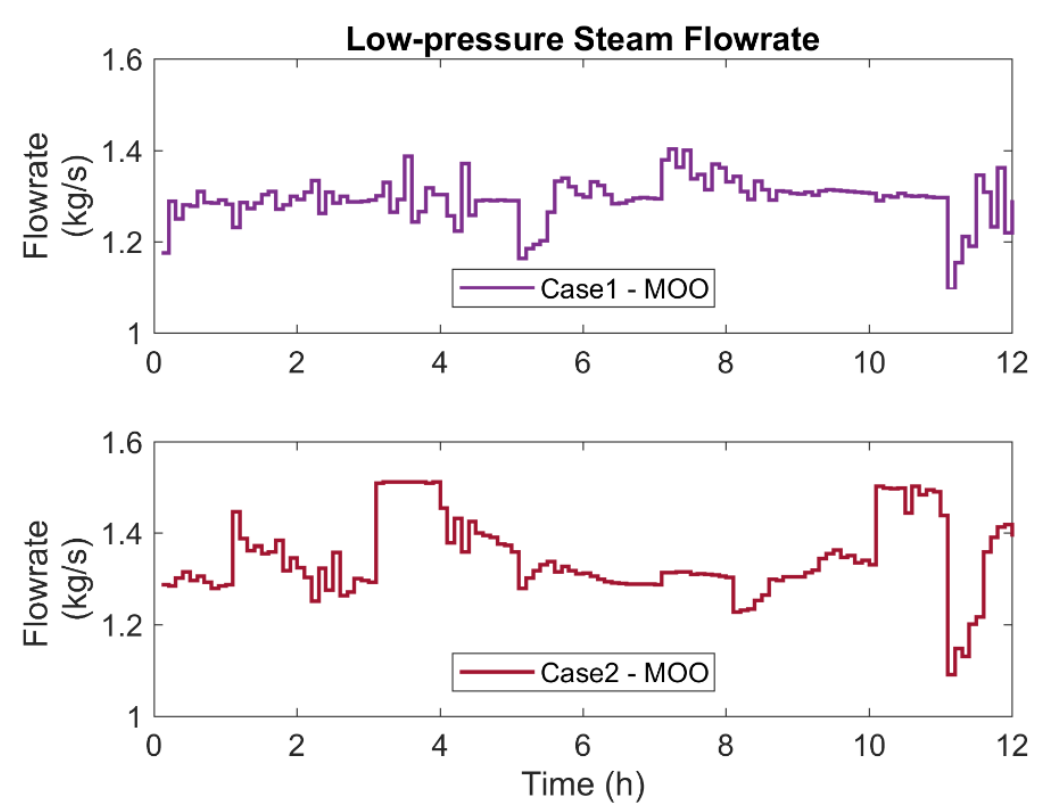

Figure 4.25. Low-pressure steam flowrate for Cases 1 and 2 obtained with MTch multi-objective optimization
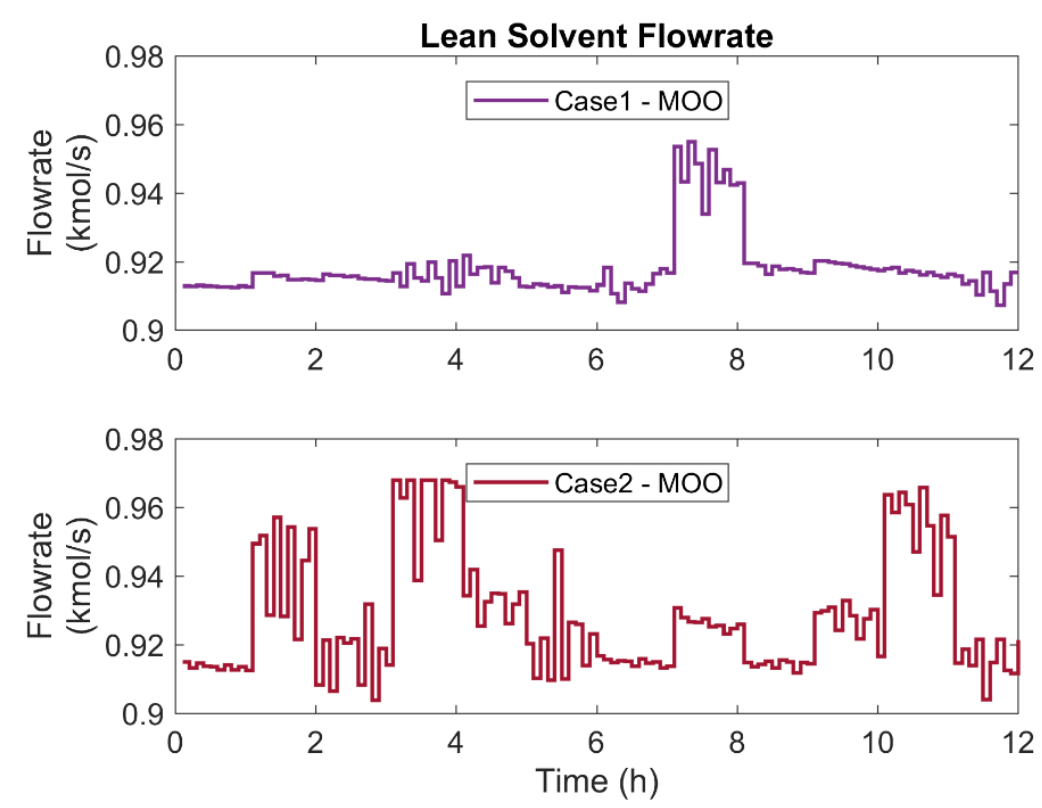

Figure 4.26. Lean solvent flowrate for Cases 1 and 2 obtained with MTch multiobjective optimization 

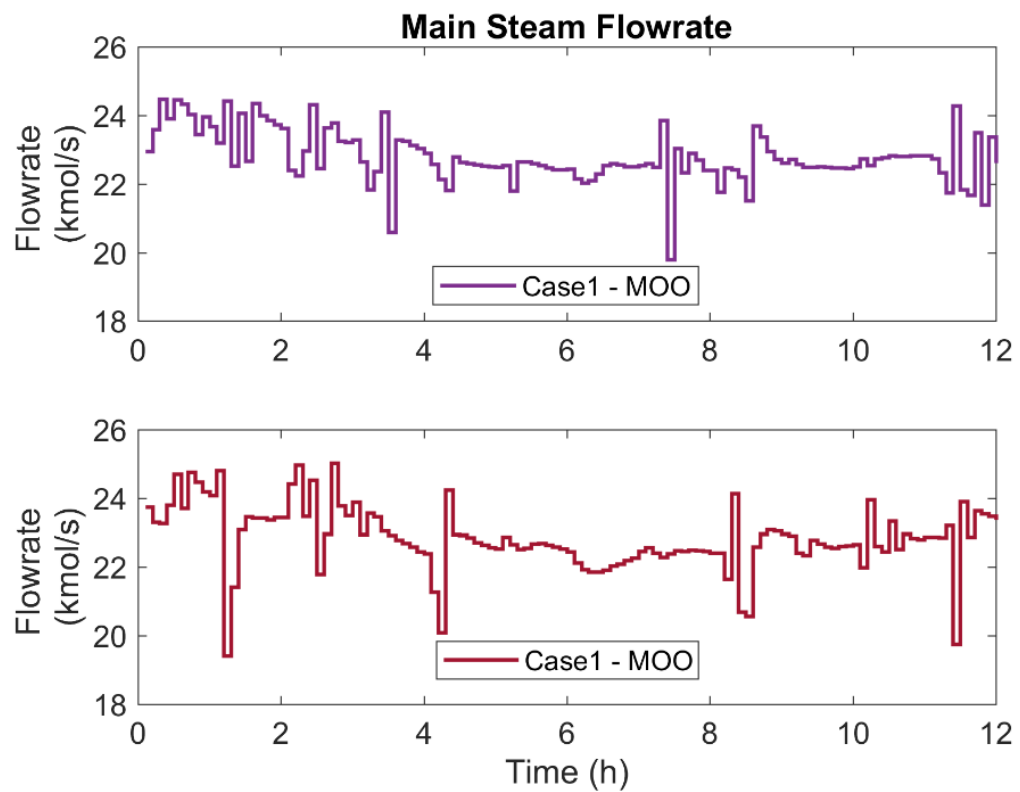

Figure 4.27. Main steam flowrate for Cases 1 and 2 obtained with MTch multiobjective optimization
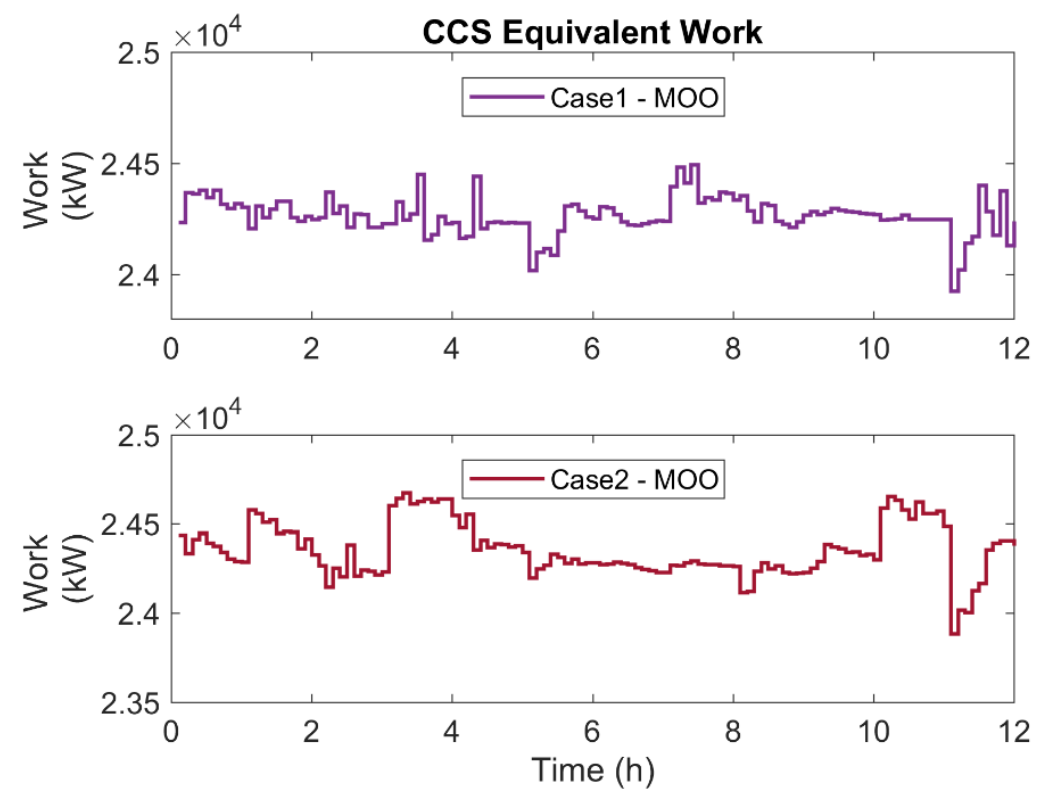

Figure 4.28. CCS equivalent work for Cases 1 and 2 obtained with MTch multiobjective optimization 

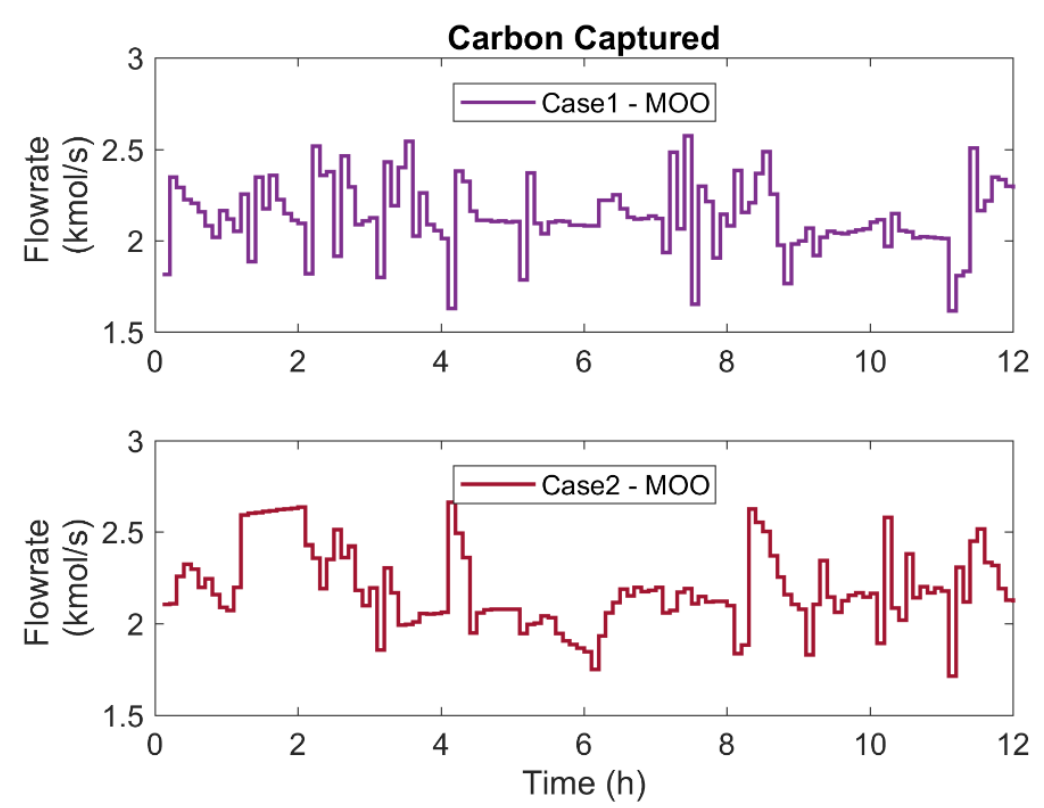

Figure 4.29. Carbon captured for Cases 1 and 2 obtained with MTch multi-objective optimization
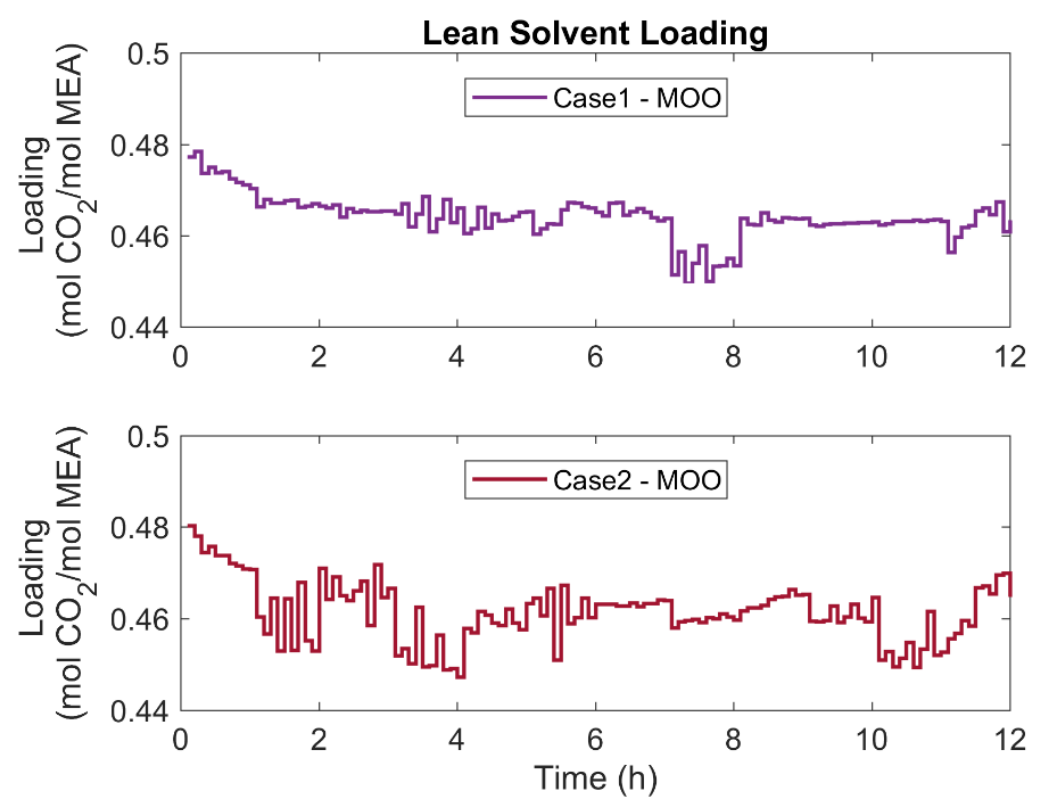

Figure 4.30. Lean solvent $\mathrm{CO}_{2}$ loading for Cases 1 and 2 obtained with MTch multiobjective optimization 
The MOO output trajectories confirm that carbon economic incentives influenced in the compromise selection. There is an overall increase of low-pressure steam extracted from the turbines, higher CCS equivalent work, and higher amount of carbon captured in Case 2 when compared to Case 1 . The lean solvent $\mathrm{CO}_{2}$ loading and the main steam flowrate trajectories remain relatively similar for both cases. Additionally, the efficiencies of the low-pressure turbines are always higher than $85 \%$. The trajectories obtained by this methodology is derived from an open-loop simulation. Therefore, for real world application, it is recommended pairing the NMPO with a multivariable controller to close the loop by sending the plant state back to the NMPO, such as in the open literature studies (Jamaludin and Swartz, 2017; Li and Swartz, 2018).

Regarding the $\epsilon$-constraint-based NPMO, a challenge is faced as a "bang-bang" effect is detected during its implementation. Considering that the NMPO has two horizons, it is not desired for the generated trajectory to satisfy the objectives separately. Figure 4.31 illustrates the "bang-bang" impact in the NMPO framework. Example 1 depicts a constant trajectory and Example 2 depicts a trajectory that starts close to zero which later rapidly increases to a high value. Both trajectories generated for the optimization horizon ( $\left.\mathrm{t}_{2}\right)$ have similar integral values. However, in the NMPO framework only the trajectory up to the implementation horizon $\left(t_{1}\right)$ is applied to the system.

Therefore, a trajectory equivalent to Example 1 is preferred over the trajectory in Example 2 for the NMPO framework, as it remains balanced at all times. A behavior equivalent to Example 1 is detected in the modified Tchebycheff-based NMPO formulation. However, a behavior equivalent to Example 2 is detected in carbon captured trajectories generated by the $\epsilon$-constraint-based NMPO. Due to this behavior, often the implementation objective values and trajectories were approximately equal to the economic objective for the $\epsilon$-constraint-based NMPO.

To partially address this challenge, a constraint in the carbon captured trajectory is included. This constraint states that at no time the MOO trajectory can be equal to the trajectories generated by either the economic or the environmental optimization. 


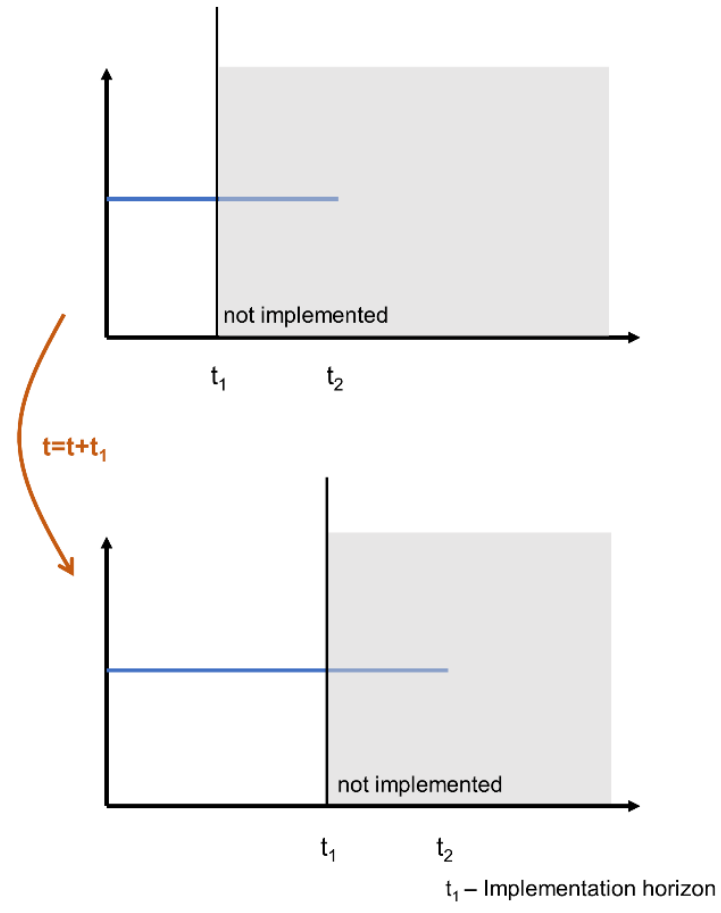

Example 1
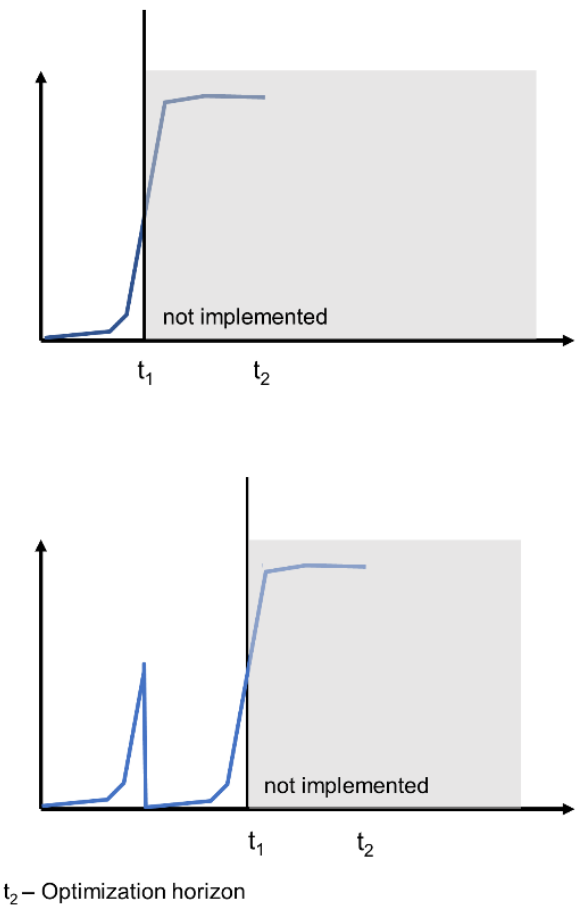

Example 2

Figure 4.31. Illustrative example of the "bang-bang" effect in the NMPO framework

For the purposes of this analysis, a shorter time horizon of $\mathrm{T}=6 \mathrm{~h}$ is performed for the $\epsilon$-constraint-based MOO. Figures 4.32 to 4.39 show the objective values for the optimization and implementation horizons of the economic, environmental, and the $\epsilon$-constraint-based MOO optimizations obtained using the NMPO for Cases 1 and 2. 


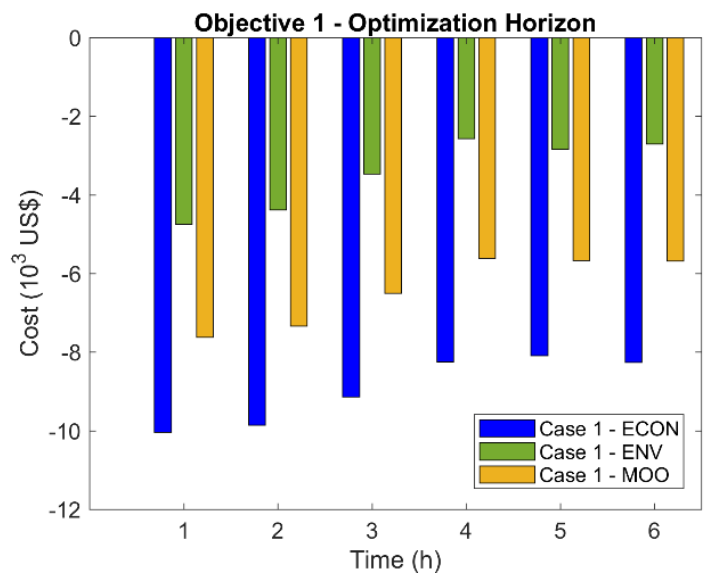

Figure 4.32. Economic objective value for optimization horizon of the economic, environmental, and $\epsilon$-constraint multiobjective optimizations - Case 1

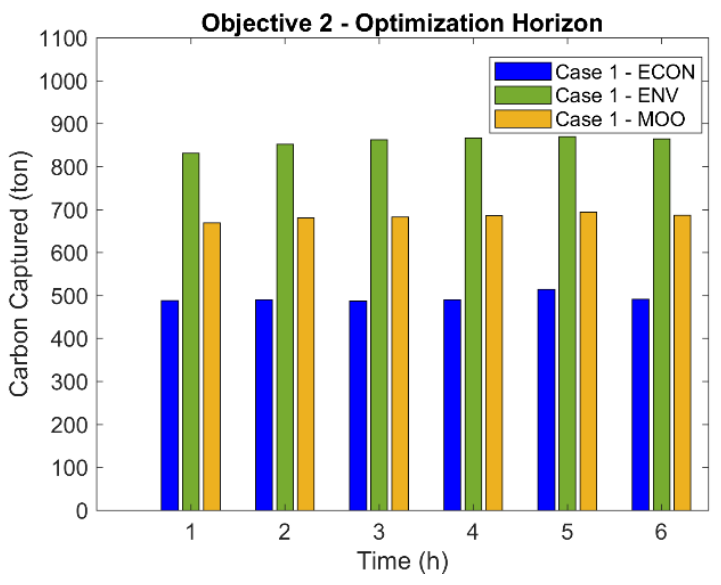

Figure 4.34. Environmental objective value for optimization horizon of the economic, environmental, and $\epsilon$ constraint multi-objective optimizations Case 1

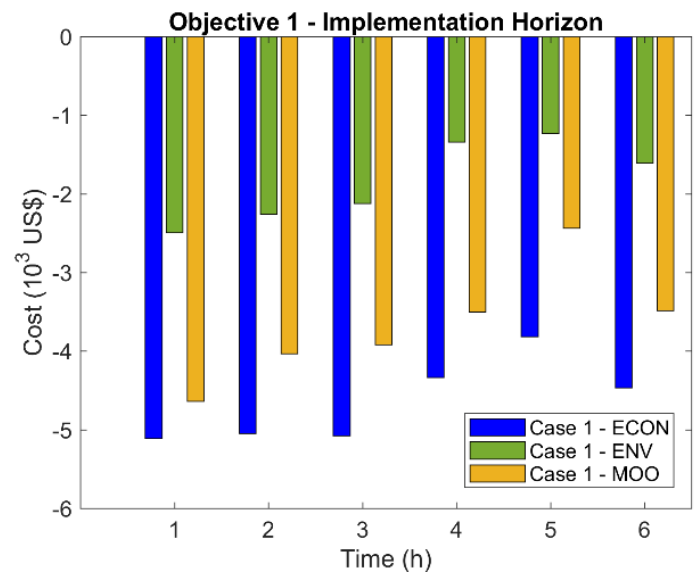

Figure 4.33. Economic objective value for implemented horizon of the economic, environmental, and $\epsilon$-constraint multiobjective optimizations - Case 1

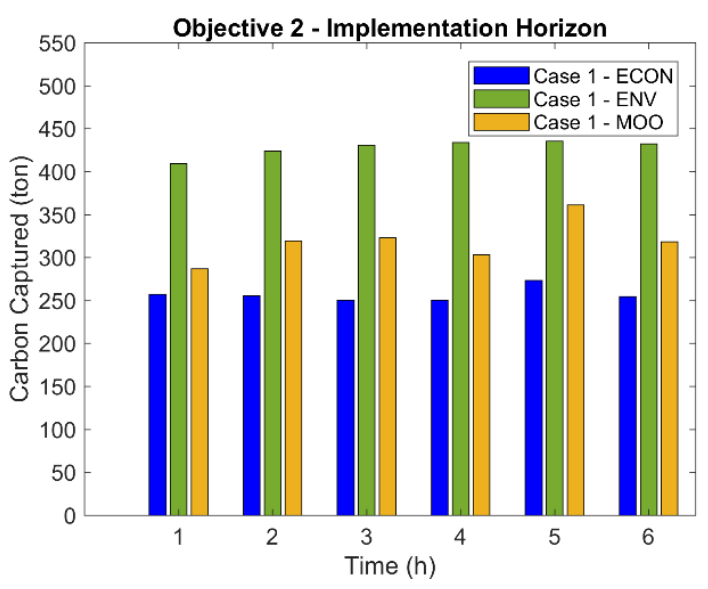

Figure 4.35. Environmental objective value for implemented horizon of the economic, environmental, and $\epsilon$ constraint multi-objective optimizations Case 1 


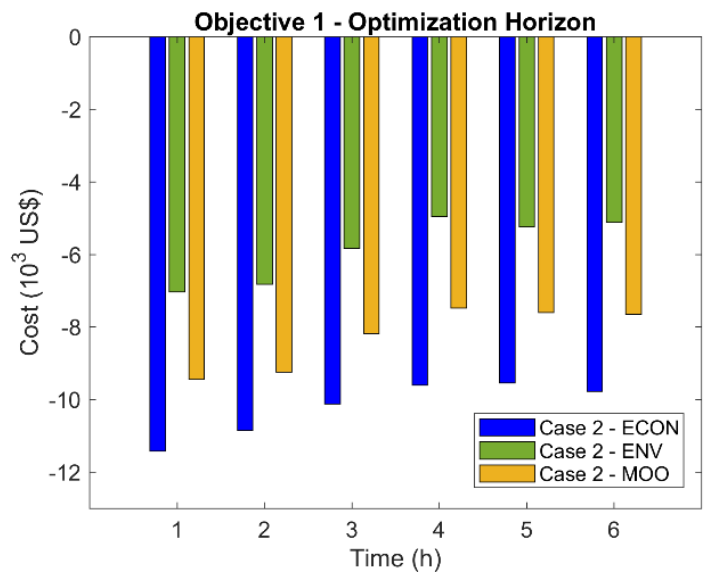

Figure 4.36. Economic objective value for optimization horizon of the economic, environmental, and $\epsilon$-constraint multiobjective optimizations - Case 2

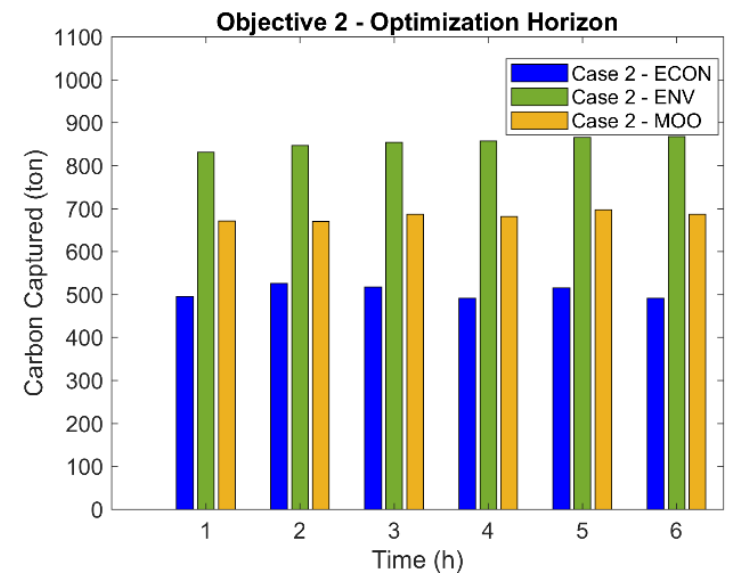

Figure 4.38. Environmental objective value for optimization horizon of the economic, environmental, and $\epsilon$ constraint multi-objective optimizations Case 2

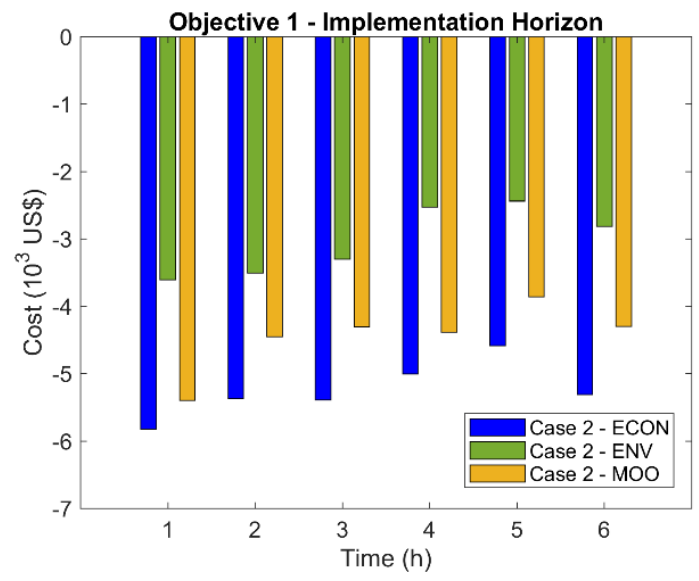

Figure 4.37. Economic objective value for implemented horizon of the economic, environmental, and $\epsilon$-constraint multiobjective optimizations - Case 2

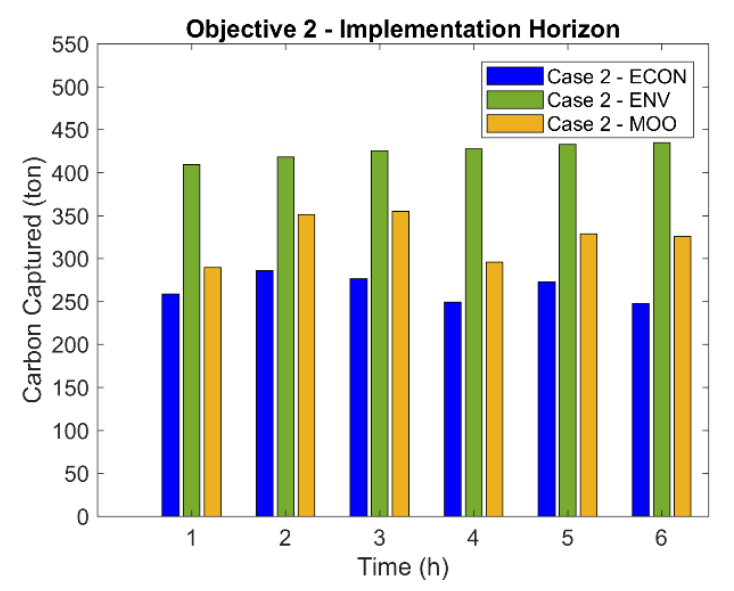

Figure 4.39. Environmental objective value for implemented horizon of the economic, environmental, and $\epsilon$ constraint multi-objective optimizations Case 2

The results show that the $\epsilon$-constraint-based MOO performs as expected for the optimization horizon (Figures 4.32, 4.34, 4.36, and 4.38), as the MOO remains in between the economic and environmental objectives. Regarding the implementation horizon (Figures $4.33,4.35,4.37$, and 4.39), the trajectory constraint can partially manage the challenge illustrated in Figure 4.31 for both performed cases. However, the objective 
values between the implemented and the optimization horizons are still not proportional, showing a bias towards the economic objective values and hindering the environmental performance.

This behavior indicates that, although the $\epsilon$-constraint-based $\mathrm{MOO}$ is a good candidate for a MOO-DRTO framework as it is capable of selecting a Pareto-based optimal compromise, the combination with the NMPO rolling horizon feature deems the performance as unsatisfactory. However, considering the performance for the optimization horizon, the $\epsilon$-constraint-based $\mathrm{MOO}$ should be explored in applications that are not updated as frequently in a rolling horizon manner, such as multi-objective scheduling and unit commitment problems. 


\section{Implementation of the MILP-based Dynamic Dispatch considering Power Plant Health}

In this chapter, the details of the MILP-based dynamic dispatch implementation considering power plant health are provided. Particularly, brief descriptions of the energy systems derived linear reduced-order models are given. Then the optimization results are presented.

\subsection{Energy Systems Dynamic Modeling}

The energy systems are linearized models from the high-fidelity models of the standalone supercritical coal-fired power plant (SCPC) (Zhang et al., 2016), charge and discharge models of the sodium sulfur (NaS) batteries (Schaefer et al., 2020), and the natural-gas combined cycle power plant (NGCC) (Wang et al., 2020), as represented in Figures 5.1 to 5.3. The wind and solar power generation, represented in Figure 5.4, are considered as boundary conditions, i.e., forcing functions based on historical data (PJM, 2019). Thus, the dispatchable energy set (ES) is defined as $E S=\{N a S, N G C C, S C P C\}$.

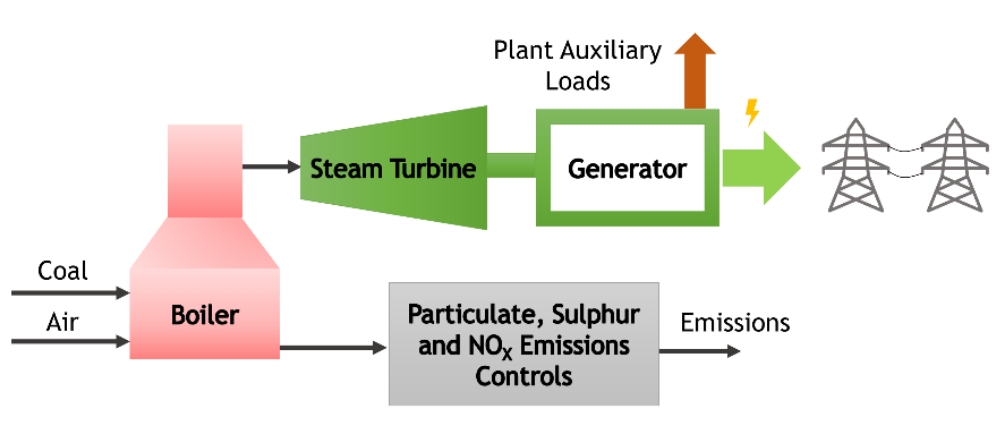

Figure 5.1. Schematic of the SCPC power plant (adapted from Kim et al., 2020)

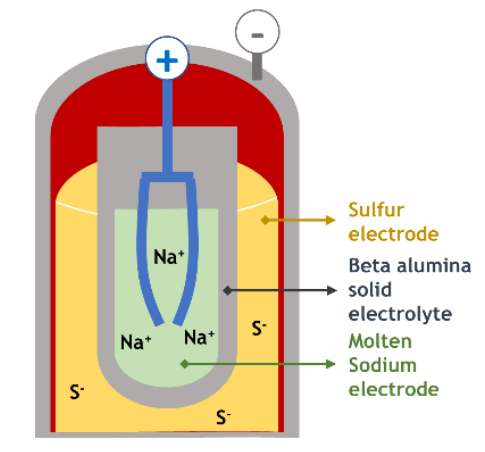

Figure 5.2. Schematic of a single NaS cell (adapted from Kim et al., 2020) 


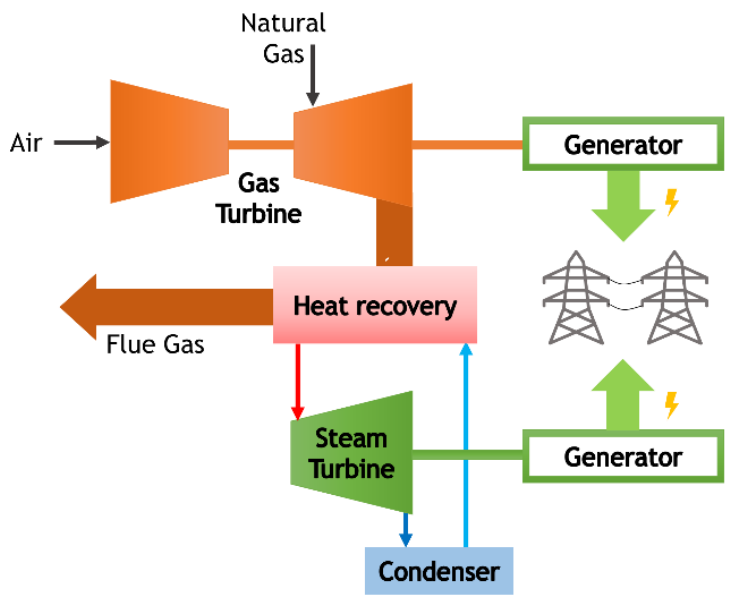

Figure 5.3. Schematic of the NGCC power plant (adapted from Kim et al., 2020)

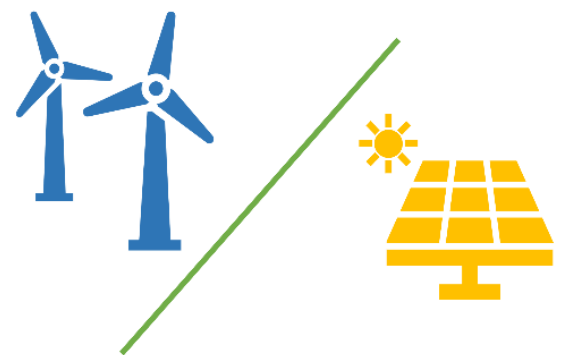

Figure 5.4. Representation of the wind and solar (adapted from Kim et al., 2020)

The data used to obtain the parameters for the linear models of the NaS batteries for charge and discharge cycles are shown in Figures 5.5 and 5.6.

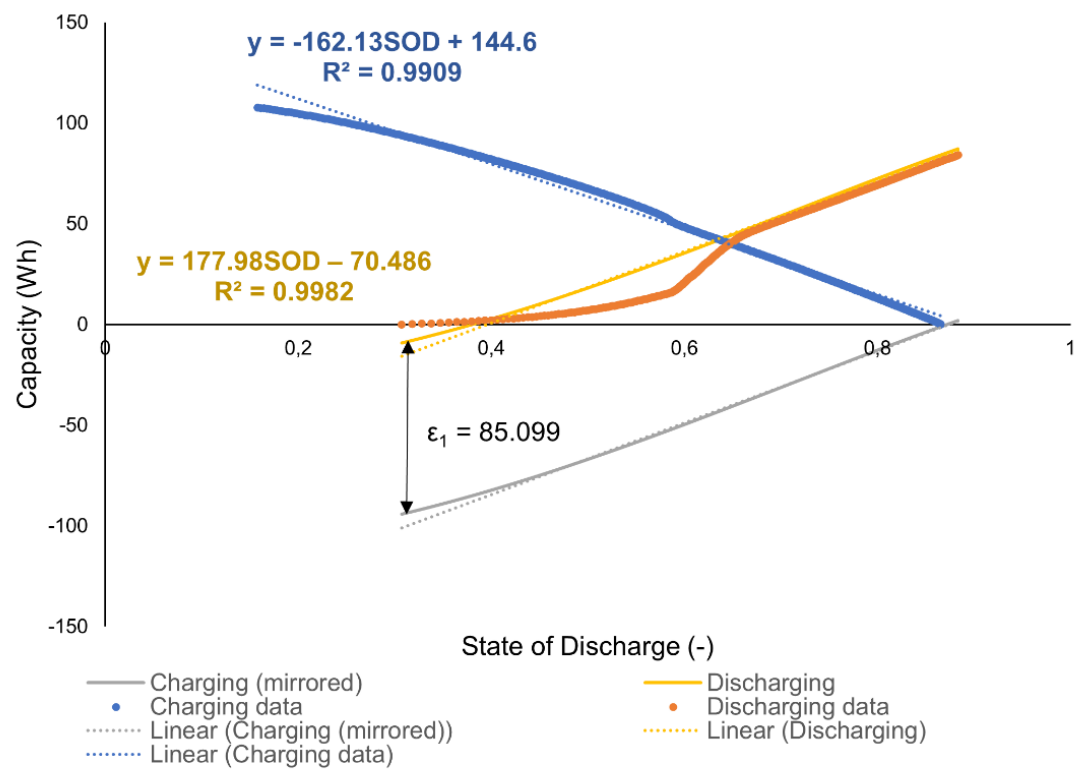

Figure 5.5. Plot of capacity (Wh) vs. state of discharge of a single NaS cell 


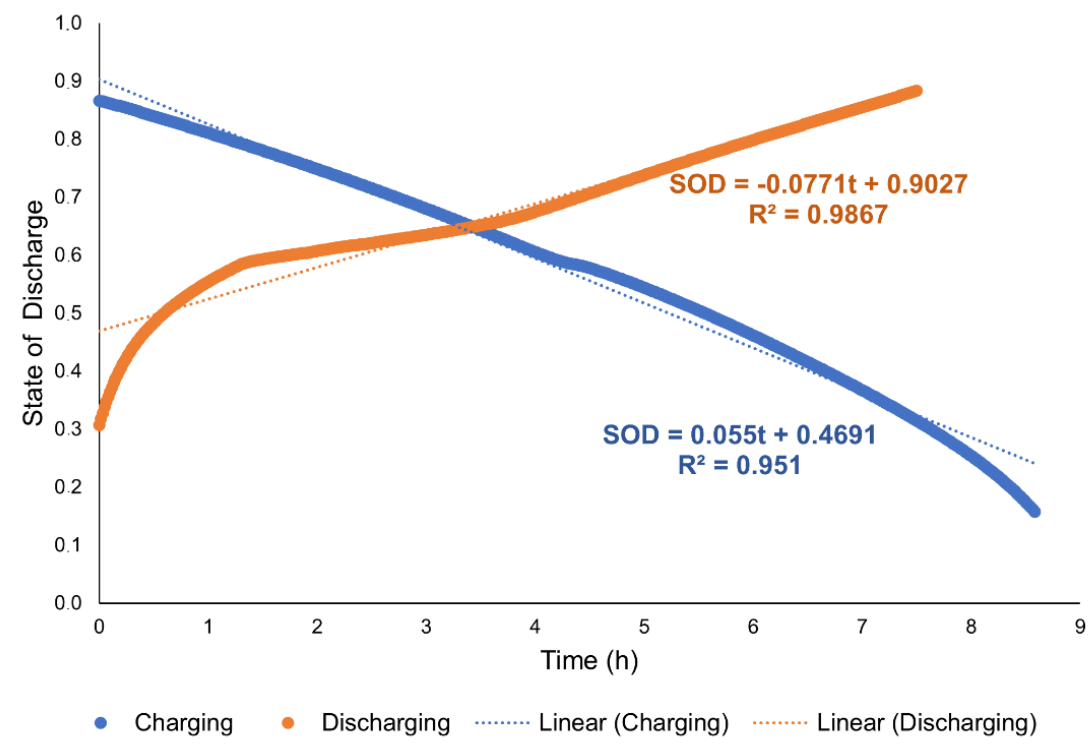

Figure 5.6. Plot of state of discharge vs. time for a single NaS cell

The NaS battery linear charging model parameters expressed in Equations (5.1) and (5.2) are calculated by linear regression of the charging data obtained by exciting the highfidelity model in Aspen Custom Modeler (Schaefer et al., 2020).

$$
\begin{aligned}
S O D & =0.055 t+0.46910 \\
\text { Capacity } & =-162.13 \text { SOD }+144.6
\end{aligned}
$$

SOD is the state of discharge (fractional), $t$ is the time in hour, and Capacity is the battery capacity in Wh.

The NaS battery linear discharging model parameters expressed in Equations (5.3) and (5.4) are calculated by considering the charging data and adding a deviation term $\left(\varepsilon_{1}\right)$ to shift the model predictions for alignment with the discharging data. The discharging data was also obtained from the high-fidelity model in Aspen Custom Modeler (Schaefer et al., 2020). A limitation in the linear representation of the discharging model is observed regarding the mismatch between the prediction and the data at lower levels of SOD. This issue arises due to the inherent phase change characteristics of $\mathrm{NaS}$ batteries during charging/discharging that alter their dynamic behavior (McKubre et al., 1989). 


$$
\begin{gathered}
S O D=-0.0771 t+0.9027 \\
\text { Capacity }=177.98 \text { SOD }-70.486
\end{gathered}
$$

The calculated $\mathrm{R}^{2}$ considering all of the linear regressions performed for the $\mathrm{NaS}$ batteries were higher than $95 \%$.

Regarding the NGCC, the reduced-order model (ROM) is obtained by linearization of a nonlinear high-fidelity model in Aspen Dynamics (Wang et al., 2020). The ROM is a statespace model as shown in Equations (5.5) and (5.6):

$$
\begin{gathered}
x_{N G C C}(t+1)=A_{N G C C} x_{N G C C}(t)+B_{N G C C} u_{N G C C}(t) \\
y_{N G C C}(t)=C_{N G C C} x_{N G C C}(t)+D_{N G C C} u_{N G C C}(t)
\end{gathered}
$$

in which, $y_{N G C C}$ is the output vector, $u_{N G C C}$ is the input vector, $x_{N G C C}$ is the state vector, and $\left(A_{N G C C}, B_{N G C C}, C_{N G C C}, D_{N G C C}\right)$ are the NGCC system matrices. The NGCC input is the natural gas flowrate $(\mathrm{kg} / \mathrm{h})$ and the outputs are the gross power output (MWe), gas turbine power output $(\mathrm{MWe})$, the main steam temperature $\left({ }^{\circ} \mathrm{C}\right)$, the reheat steam temperature $\left({ }^{\circ} \mathrm{C}\right)$, the drum body mechanical stress (MPa), and the thermal drum body stress (MPa). In summary, the mechanical and thermal stresses calculated in the high-fidelity model are a function of the material of construction, geometry of the equipment, operating pressure and temperature. During the optimization, considering the linear programming constraint, only the most stressed direction was considered in this work, i.e., the tangential direction.

The reduced-order linear model for the SCPC, obtained by linear regression using the generated data from the high-fidelity model in Aspen Dynamics (Zhang et al., 2016), is an autoregressive with exogenous inputs (ARX) model, previously shown in Equation (4.6). The SCPC regressors are composed of the past and current coal feedrates $(\mathrm{kg} / \mathrm{s})$ and the output vector contains the past and current gross power outputs (kWe). The regressors for the SCPC ARX model are shown in Equations (5.7) and (5.8).

$$
\begin{gathered}
y(t)=[\text { power output }(t, t-1, t-2, t-3)] \\
x(t)=[\text { coal feedrate }(t, t-1)]
\end{gathered}
$$


Figures 5.7 to 5.13 show the correspondence between the model predictions and the generated datasets from the high-fidelity models. For the NGCC models, FOM is the fullorder model, ROM is the reduced-order model, and APD is the Aspen Plus Dynamic data. Linearization of the high-fidelity nonlinear models inherently introduces inaccuracies in the representation of the energy systems. In particular, the mechanical stress is underestimated at full load, and the thermal stress is underestimated at peaks. However, the overall dynamic behavior of the integrated energy system is still captured by the reduced-order model.
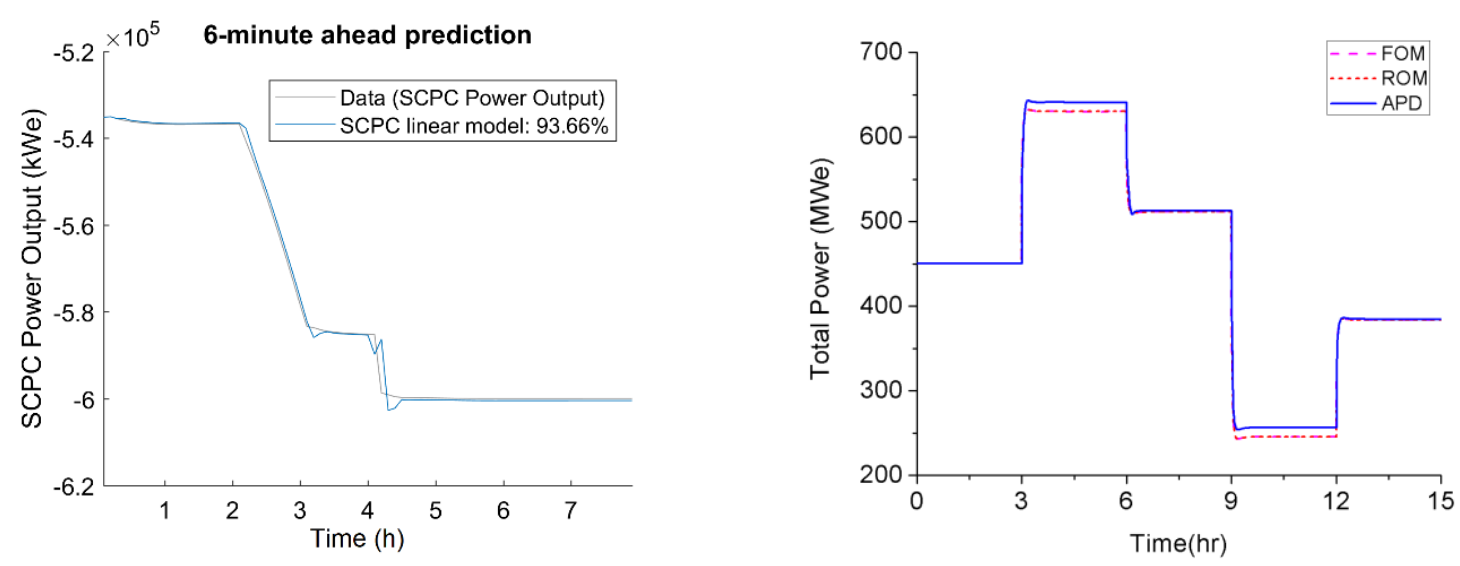

Figure 5.7. Comparison between 6

minute ahead model prediction with data from high-fidelity Aspen model - SCPC total power output

Figure 5.8. Comparison between simulations and data from high-fidelity Aspen model - NGCC total power output 


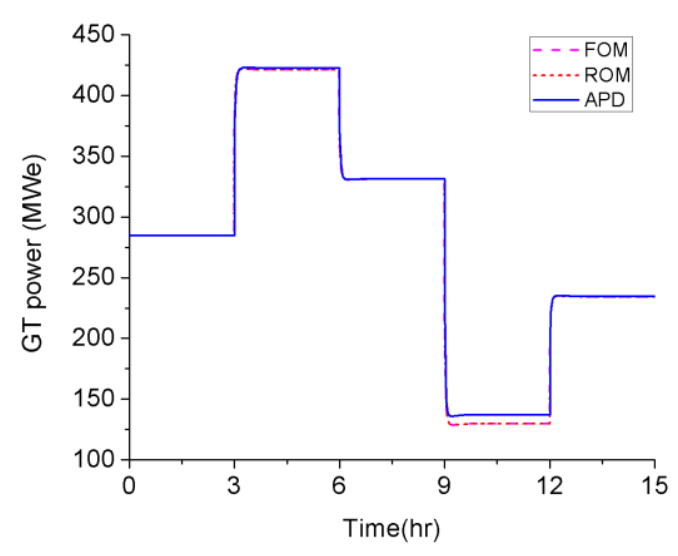

Figure 5.9. Comparison between simulations and data from high-fidelity Aspen model - NGCC gas turbine power output

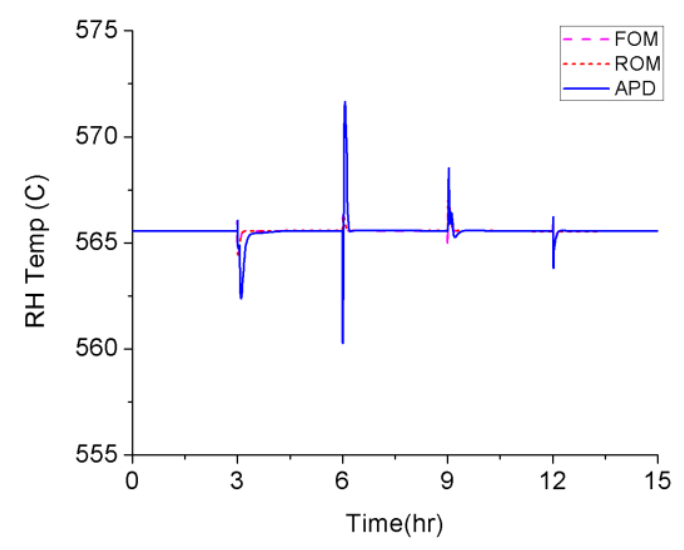

Figure 5.11. Comparison between simulations and data from high-fidelity Aspen model - NGCC gas reheat steam temperature

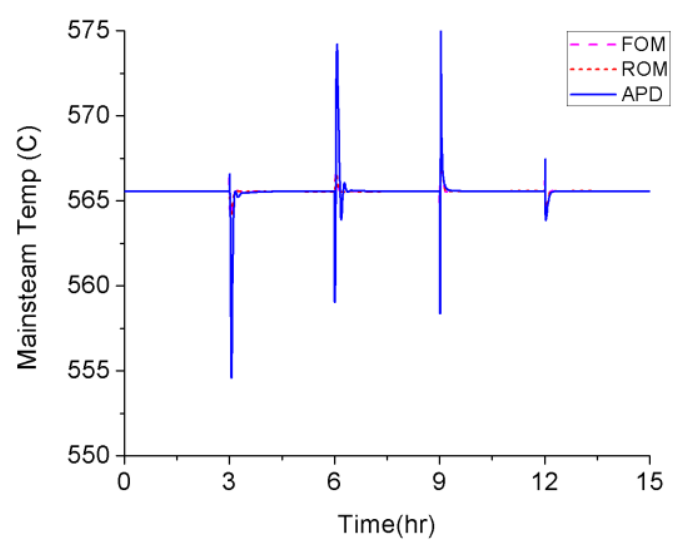

Figure 5.10. Comparison between simulations and data from high-fidelity Aspen model - NGCC main steam temperature

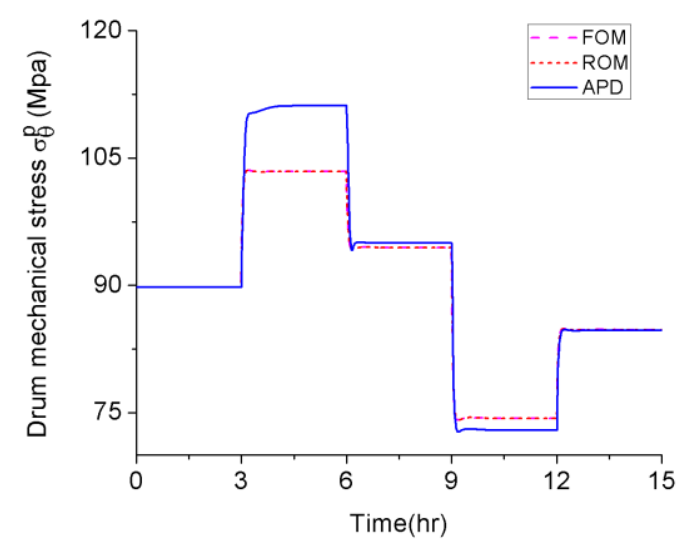

Figure 5.12. Comparison between simulations and data from high-fidelity Aspen model - NGCC drum mechanical stress 


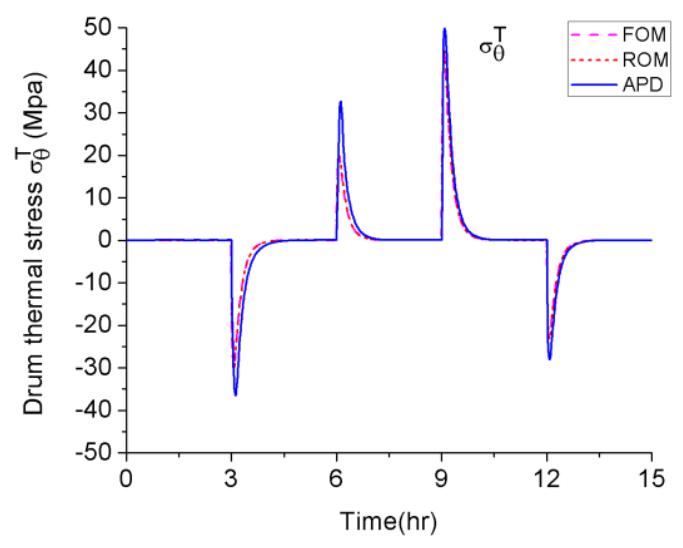

Figure 5.13. Comparison between simulations and data from high-fidelity Aspen model - NGCC drum thermal stress

\subsection{Dynamic Dispatch Optimization}

\subsubsection{Problem Statement}

The objective of the dynamic dispatch optimization is to minimize the overall cost of dispatch defined in Equation (5.9):

$$
\min \Phi=\text { Cost }_{E S}-\text { Revenue }_{E S}
$$

in which ES represents the dispatchable energy set in the grid. The "Cost Es" and "RevenueEs" variables are further defined in Equations (5.10) and (5.11).

$$
\begin{gathered}
\text { Cost }_{E S}=\text { Variable operation and maintenance }_{E S}+\text { Cost of fuel } \\
\text { Penalty }_{E S}+ \\
\text { Pevenue }_{E S}=\text { Wholesale of generated power } \\
\text { RevS }_{\text {ensumption }},
\end{gathered}
$$

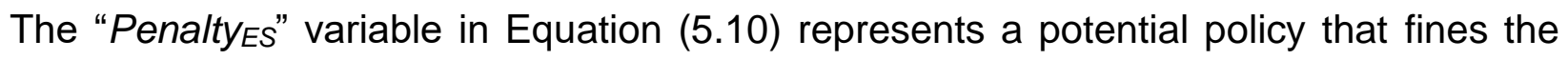
curtailment of renewables or the investment cost per cycle of the battery. The nominal power generation for the NGCC is $641 \mathrm{MWe}$, for the SCPC is $600 \mathrm{MWe}$, and for the NaS batteries is $99.4 \mathrm{MW} / 845 \mathrm{MWh}$ of storage capacity considering a single sodium sulfur cell capacity equal to $102 \mathrm{Wh}$. The maximum discharge and charge rates of a single NaS cell are assumed to be $12.5 \mathrm{Wh}$ and $8.9 \mathrm{Wh}$, respectively. Furthermore, the optimization problem statement also includes the constraints expressed in Equations (5.12) to (5.23). 
The smart-grid power balance is expressed in Equation (5.12).

$$
\begin{gathered}
\sum_{i \in E S}\left(\text { Electricity produced }_{i}-\text { Electricity consumed }_{i}\right)+\text { Renewables } \\
=\text { Demand }
\end{gathered}
$$

The "Electricity consumed" variable refers to the case in which the energy system requires electricity from the grid that cannot be supplied from within the energy system (for instance, when the battery requires electricity to charge). The "Renewables" variable considers the wind and solar power generation as forcing function profiles built from PJM Regional Transmission Organization data (PJM, 2019). The data corresponds to 30 days of hourly aggregate demand loads and the electricity price in August 2019, which were linearly interpolated for the 6 min time step. The absolute values of the demand and the renewable power generation are adjusted to be proportional to the available energy sources' nominal maximum and minimum power generations, while maintaining the load demand slopes. These actions are taken to guarantee feasibility to the current forcing functions that use 2019 information. However, further modifications to the forcing functions (the modified 2019 load and the 2050 renewables projection as shown in Figures 5.14 and 5.15) might render the problem infeasible due higher energy supply than demand. Thus, to partially manage this issue, renewables curtailment is allowed and flexibility requirements of the grid analyzed. The proportionality factors used for the scaling of renewable generation in 2050 are based on information in Table 5.1.

Table 5.1. Electricity generation from selected fuels (EIA, 2019a)

\begin{tabular}{|c|c|c|}
\hline Fuel & $\mathbf{2 0 1 8}$ & $\mathbf{2 0 5 0}$ \\
\hline Natural gas & $34 \%$ & $39 \%$ \\
\hline Coal & $28 \%$ & $17 \%$ \\
\hline Renewables & $18 \%$ & $31 \%$ \\
\hline Wind & $6.66 \%$ & $7.77 \%$ \\
\hline Solar & $2.34 \%$ & $14.88 \%$ \\
\hline Other ${ }^{1}$ & $9.00 \%$ & $8.35 \%$ \\
\hline Nuclear & $19 \%$ & $12 \%$ \\
\hline Total & $99 \%$ & $99 \%$ \\
\hline
\end{tabular}

${ }^{1}$ Other renewable sources, e.g., hydro and geothermal. 
Figures 5.14 and 5.15 show the power demands and renewable penetration levels based considered for most of the case studies.

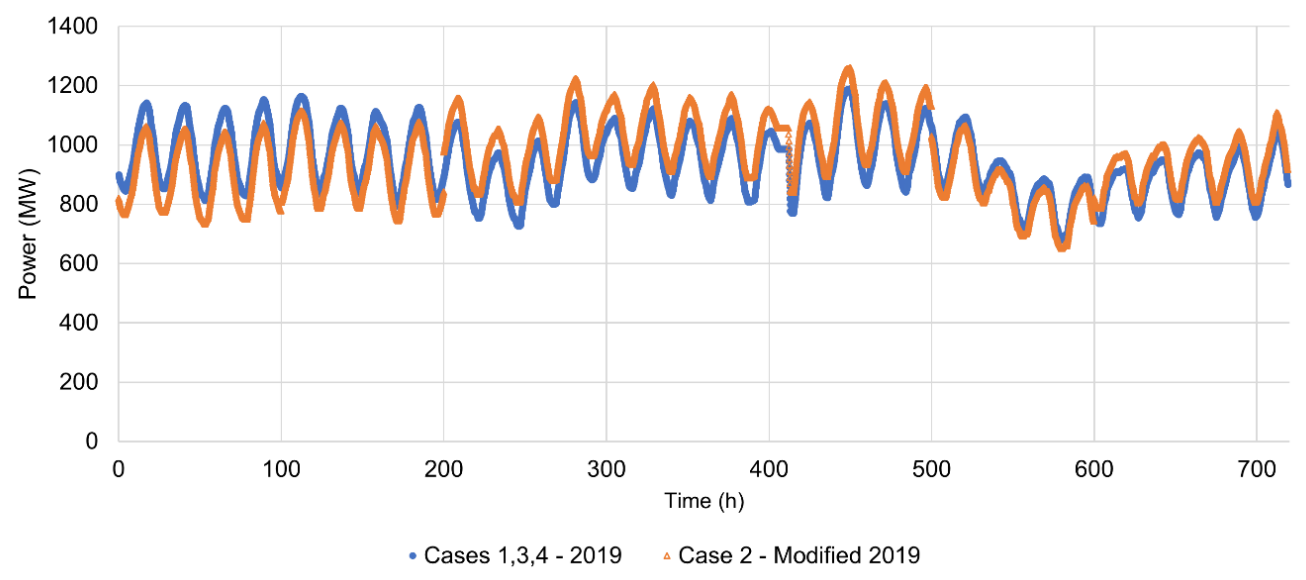

Figure 5.14. 2019 total and modified electricity demand load

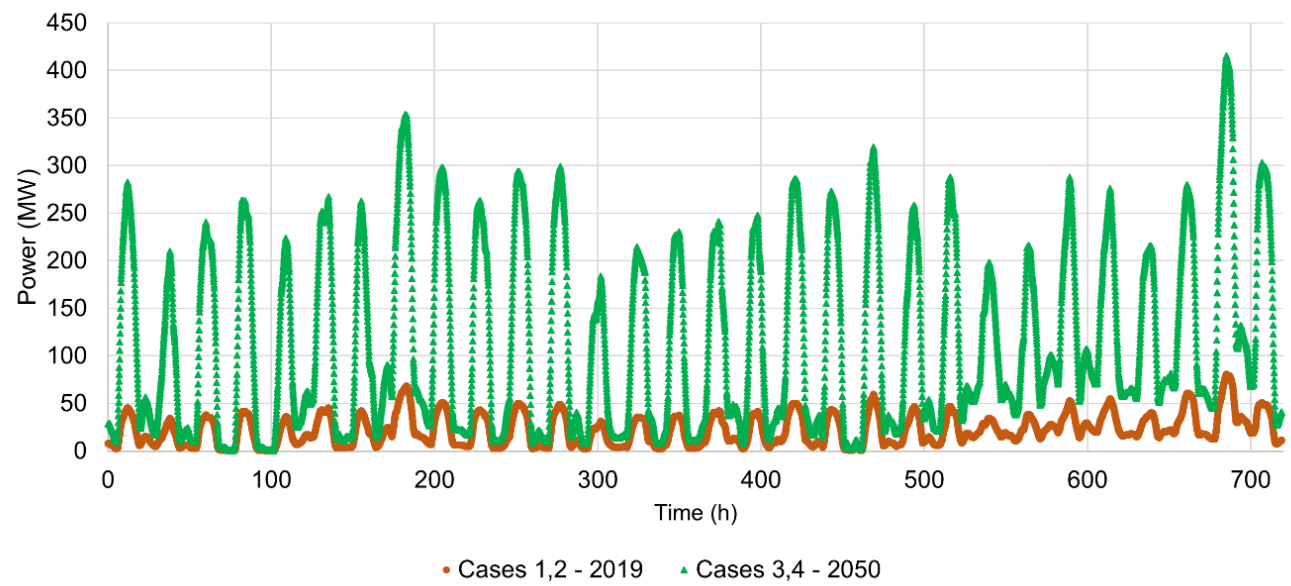

Figure 5.15. 2019 and 2050 variable renewable energy penetrations (solar/wind)

A logical constraint using disjunctive programming is also included, as it is considered that the NaS batteries cannot charge and discharge at the same time. This constraint is expressed in Equations (5.13) and (5.14).

$$
\begin{gathered}
v \in \mathbb{Z} \mid v_{\text {discharge }}+v_{\text {charge }} \leq 1, v \in\{0,1\} \\
v_{\text {discharge/charge }}=\left\{\begin{array}{lr}
0 & \text { discharge } / \text { charge model not selected } \\
1 & \text { discharge } / \text { charge model selected }
\end{array}\right.
\end{gathered}
$$


In summary, the decision variables for the dynamic dispatch are the SCPC coal feedrate, the NGCC natural gas flowrate, the two integer variables $v_{\text {discharge/charge, }}$, the level of renewables curtailment, and the depreciation of the battery.

The NaS batteries range of operation considering the regression data fit is shown in Equation (5.15).

$$
40 \% \leq S O D \leq 89 \%
$$

Finally, the damage-related constraints of the drum, the superheater, and reheater are also included. In summary, two distinct wear and tear mechanisms can influence and change the material lifetime, namely creep and fatigue. Creep is caused by the prolonged exposure to high temperature, while fatigue is due to the repeated and fluctuating stress that results from both temperature and pressure transients under load-following conditions (Kim et al., 2010).

In the optimization problem, the maximum stress that is allowed for the drum is considered as a constraint along with a constraint on the maximum temperature to avoid overheating of the NGCC superheater and reheater. Stress analysis of the NGCC drum was based on the European norm (European Committee for Standardization, 2002), design rules for steam boilers (Technical Rules for Steam Boilers, 1996), and available literature (Kim et al., 2010). The dimensions of the drum main body and branches are shown in Table 5.2.

Table 5.2. High-pressure drum specification with $\mathrm{MOC}^{1}$ being SA- 515 grade 70

\begin{tabular}{|c|c|c|c|}
\hline Parameter & Symbol & Value & Unit \\
\hline Branch radius & $r_{\text {inb }}$ & 0.20 & $\mathrm{~m}$ \\
\hline Main body radius & $r_{\text {inms }}$ & 0.8382 & $\mathrm{~m}$ \\
\hline Branch thickness & $s_{b}$ & 0.04 & $\mathrm{~m}$ \\
\hline Main body thickness & $s_{m s}$ & 0.1524 & $\mathrm{~m}$ \\
\hline Thermal transmittance for steam & $h$ & 1000 & $\mathrm{Wm}^{-2} \mathrm{~K}^{-1}$ \\
\hline
\end{tabular}

${ }^{1}$ MOC: Material of Construction.

The tangential stress in the drum body is defined as a sum of the mechanical and thermal stresses, as shown in Equation (5.16) and it is used to represent the limiting stress as it is the highest stress component. 


$$
\sigma_{\theta, b o d y}=\sigma_{\theta, b o d y}^{P}+\sigma_{\theta, b o d y}^{T}
$$

In which, $\sigma_{\theta, b o d y}$ is the overall tangential stress on the body of the drum, $\sigma_{\theta, b o d y}^{P}$ is the tangential stress due to pressure (mechanical stress), and $\sigma_{\theta, b o d y}^{T}$ is the tangential stress due to temperature (thermal stress).

To define the threshold for the stress constraint on the body of the drum, the maximum allowable stress (MAS) is defined in Equation (5.17). Specifically, the material SA-515 grade 70 requires the tensile strength to be within the range of $485-620 \mathrm{MPa}$ and the minimum yield strength of $260 \mathrm{MPa}$ (Henan Steel Guang International Trade Co, 2020).

$$
M A S=\frac{\text { tensile } \text { strengt }_{\text {min }}}{4}
$$

The calculated MAS value is about $120 \mathrm{MPa}$. The MAS value is used to determine the maximum tangential stress value during dispatch according to Equations (5.18) and (5.19):

$$
\begin{aligned}
P_{c} & =\frac{2 M A S}{1+\frac{2 r_{i n m s}}{s_{m s} E}} \\
\text { Threshold } & =\frac{\left(P_{c}-p_{0}\right)\left(2 r_{i n m s}+s_{m s}\right)}{2 s_{m s}} .
\end{aligned}
$$

in which $p_{0}$ is the outside pressure (MPa) and $E$ is the joint efficiency of the shell-branch connection, with $E=0.95$. Then, the constraint on the overall stress that the drum body endures is given by Equation (5.20).

$$
\sigma_{\theta, b o d y} \leq \text { Threshold }
$$

Finally, to avoid overheating of the superheater and reheater, temperature constraints given by Equations (5.21) to (5.23) are considered:

$$
\begin{gathered}
T_{\text {superheater }} \leq T_{\max } \\
T_{\text {reheat }} \leq T_{\max } \\
T_{\text {max }}=635^{\circ} \mathrm{C} .
\end{gathered}
$$

Additionally, two post-optimization analyses are carried out: the stress of the most stressed part of the drum and the equivalent $\mathrm{CO}_{2}$ emission. The post-optimization analysis of the most stressed part of the drum is performed by using Equations (5.24) to 
(5.31) along with Equation (5.16) after the optimal dispatch is determined. Equations (5.24) and (5.25) are developed by multiplying the tangential thermal and mechanical body stresses using stress concentration factors. The concentration factors were developed considering the shell-branch connections (e.g., drum-downcomer junction). A diagram of the steam drum is shown in Figure 5.16.

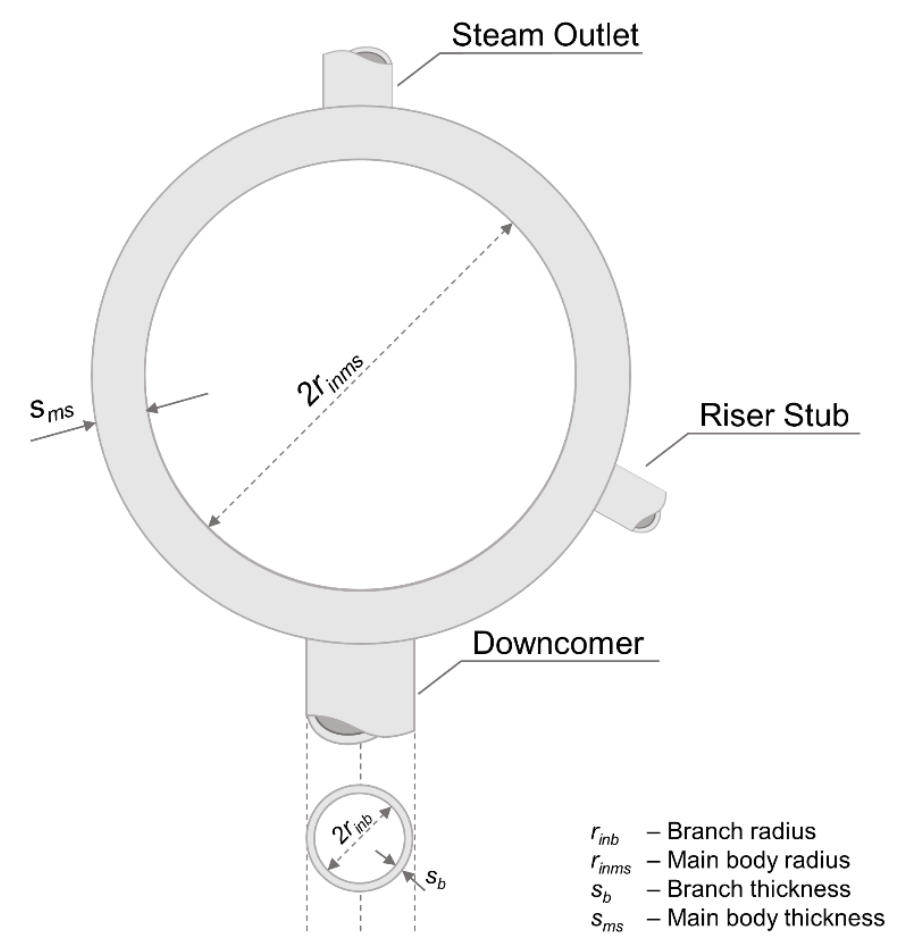

Figure 5.16. Diagram of a steam drum (including the drum-downcomer junction)

$$
\begin{gathered}
\sigma_{\theta, \text { most stressed }}^{P}=\lambda_{m} \sigma_{\theta, \text { body }}^{P} \\
\sigma_{\theta, \text { most stressed }}^{T}=\lambda_{t} \sigma_{\theta, \text { body }}^{T}
\end{gathered}
$$

In Equations (5.24) and (5.25), $\lambda_{m}$ is the stress concentration factor due to pressure and is calculated using Equations (5.26) to (5.29), while $\lambda_{t}$ is the stress concentration factor due to temperature and is calculated using Equations (5.30) to (5.31).

$$
\begin{gathered}
\lambda_{m}=2.2+e^{A} * \zeta^{B} \\
A=-1.14\left(\frac{s_{b}}{s_{m s}}\right)^{2}-0.89\left(\frac{s_{b}}{s_{m s}}\right)+1.43
\end{gathered}
$$




$$
\begin{gathered}
B=0.326\left(\frac{s_{b}}{s_{m s}}\right)^{2}-0.59\left(\frac{s_{b}}{s_{m s}}\right)+1.08 \\
\zeta=\frac{d_{m b}}{d_{m s}} \sqrt{\frac{d_{m s}}{2 s_{m s}}} \\
\lambda_{t}=\left\{\left[2-\frac{h_{t}+2700}{h_{t}+1700} \xi+\frac{h_{t}}{h_{t}+1700}\left(\exp ^{-7 \xi}-1\right)\right]^{2}+0.81 \xi^{2}\right\}^{1 / 2} \\
\xi=\frac{d_{m b}}{d_{m s}} \\
d_{m b}=2 r_{\text {inb }}+s_{b} \\
d_{m s}=2 r_{\text {inms }}+s_{m s}
\end{gathered}
$$

In Equations (5.32) and (5.33), $d_{m b}$ and $d_{m s}$ are the branch and main body mean diameters.

Regarding the environmental equivalent $\mathrm{CO}_{2}$ analysis, complete combustion of the fossilfired power plants' fuel (natural gas and coal) is assumed for the calculation of the $\mathrm{CO}_{2}$ emissions. The coal considered in this work is the bituminous Illinois no. 6 with Table 5.3 showing its composition (NETL, 2019). Table 5.4 shows the composition used for the natural gas (NETL, 2019).

Table 5.3. Bituminous Illinois No.6 coal composition

\begin{tabular}{|c|c|}
\hline Component & Weight (\%) \\
\hline Moisture & 11.12 \\
\hline Carbon & 63.75 \\
\hline Hydrogen & 4.50 \\
\hline Nitrogen & 1.25 \\
\hline Chlorine & 0.15 \\
\hline Sulfur & 2.51 \\
\hline Ash & 9.70 \\
\hline Oxygen & 7.02 \\
\hline
\end{tabular}


Table 5.4. Natural gas composition

\begin{tabular}{|c|c|}
\hline Component & Volumetric (\%) \\
\hline Methane & 93.1 \\
\hline Ethane & 3.2 \\
\hline Propane & 0.7 \\
\hline n-Butane & 0.4 \\
\hline Carbon Dioxide & 1.0 \\
\hline Nitrogen & 1.6 \\
\hline Methanethiol(A) & 0.00000575 \\
\hline
\end{tabular}

\subsubsection{Optimization Results}

The optimization problem is solved for different renewable power generations and power grid components. In summary, five case studies are simulated. Initially, four case studies consider the dispatch of NGCC, SCPC, and NaS batteries to accommodate different levels of renewable penetrations into the grid, while supplying the demand. Figures 5.14 and 5.15 show the power demands and renewable penetration levels considered in these four case studies. Then, for the fifth case study, a more challenging renewable penetration scenario is designed and an in-depth stress analysis is performed.

A technology-invariant curtailment penalty of $110 \mathrm{US} \$ / \mathrm{MWh}$ based on reference (Schill et al., 2017) is considered in all case studies. The main purpose of this penalty is to maintain the curtailment at minimum levels and the wind/solar supply is only curtailed when the lack of curtailment renders the dispatch infeasible due to oversupply of energy. The battery lifecycle is assumed to be relatively much shorter than the lifetime of the power plants, as it depends on the degradation and the number of cycles. In this study, a penalty term is considered in the objective to take into account each cycle completed by the $\mathrm{NaS}$ batteries with a cost of $300 \mathrm{US} \$ / \mathrm{kWh}$ and a maximum number of 2500 cycles. A complete charge/discharge of the battery is considered as a cycle. The costs of fuel for the SCPC and NGCC are considered constant in the timespan of 30 days and equal to 40.20 US\$/ton for the Illinois Basin coal (EIA, 2019b) and $2.67 \mathrm{US} \$ / \mathrm{ft}^{3}$ for the natural gas (EIA, 2019c). Also, for the purposes of this simulation, the batteries are initialized at $S O D=60 \%$ to allow the battery to either charge or discharge from the initial point. 
Figures 5.17 to 5.21 show the results as stacked plots, i.e., the plot is shown as cumulative in the order of appearance of each technology (SCPC, NGCC, NGCC 2, NaS batteries, and renewables). For instance, if at some time instance a second line superimposes the first line, the energy system of the second line is not providing energy at that instance. The amount of energy being provided by a specific energy system is the subtraction between the previous and current energy system. This type of plot is chosen to rapidly examine if the power of the grid is balanced. If the line that represents the last energy source to be added matches the contour of the gray shaded area (demand), that means the power is balanced.

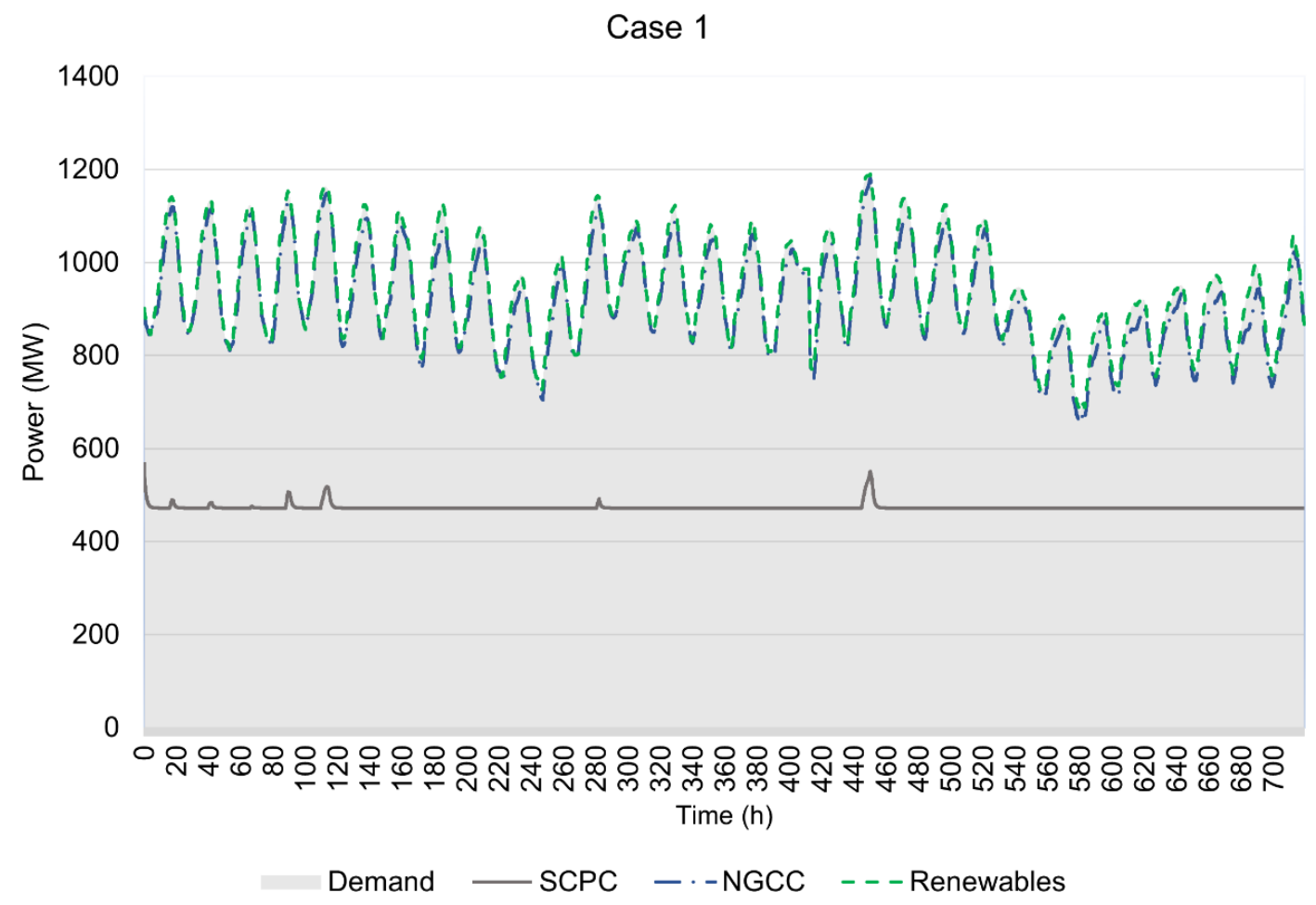

Figure 5.17. Power dispatch optimization result for Case 1 


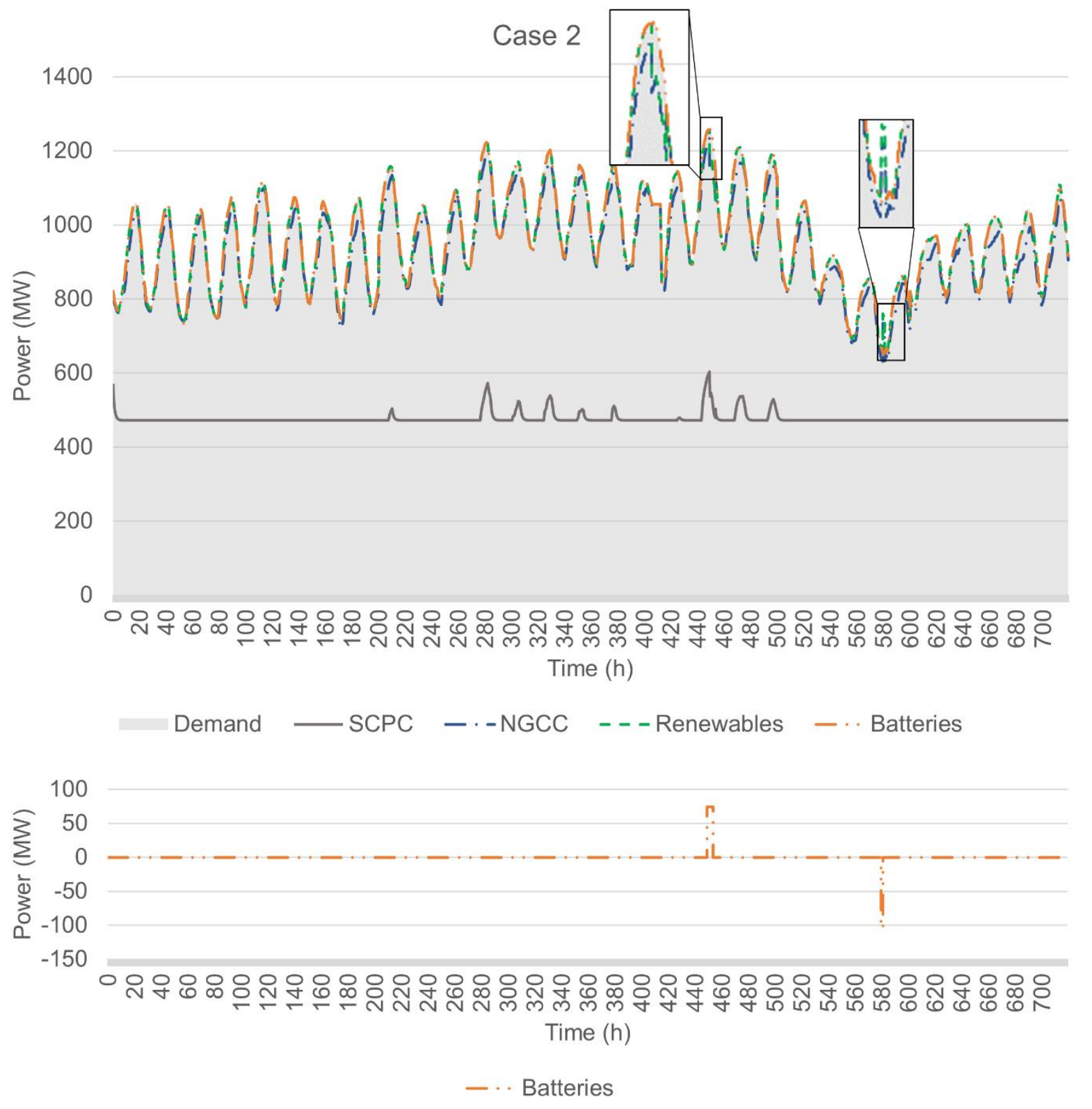

Figure 5.18. Power dispatch optimization result for Case 2 


\section{Case 3}

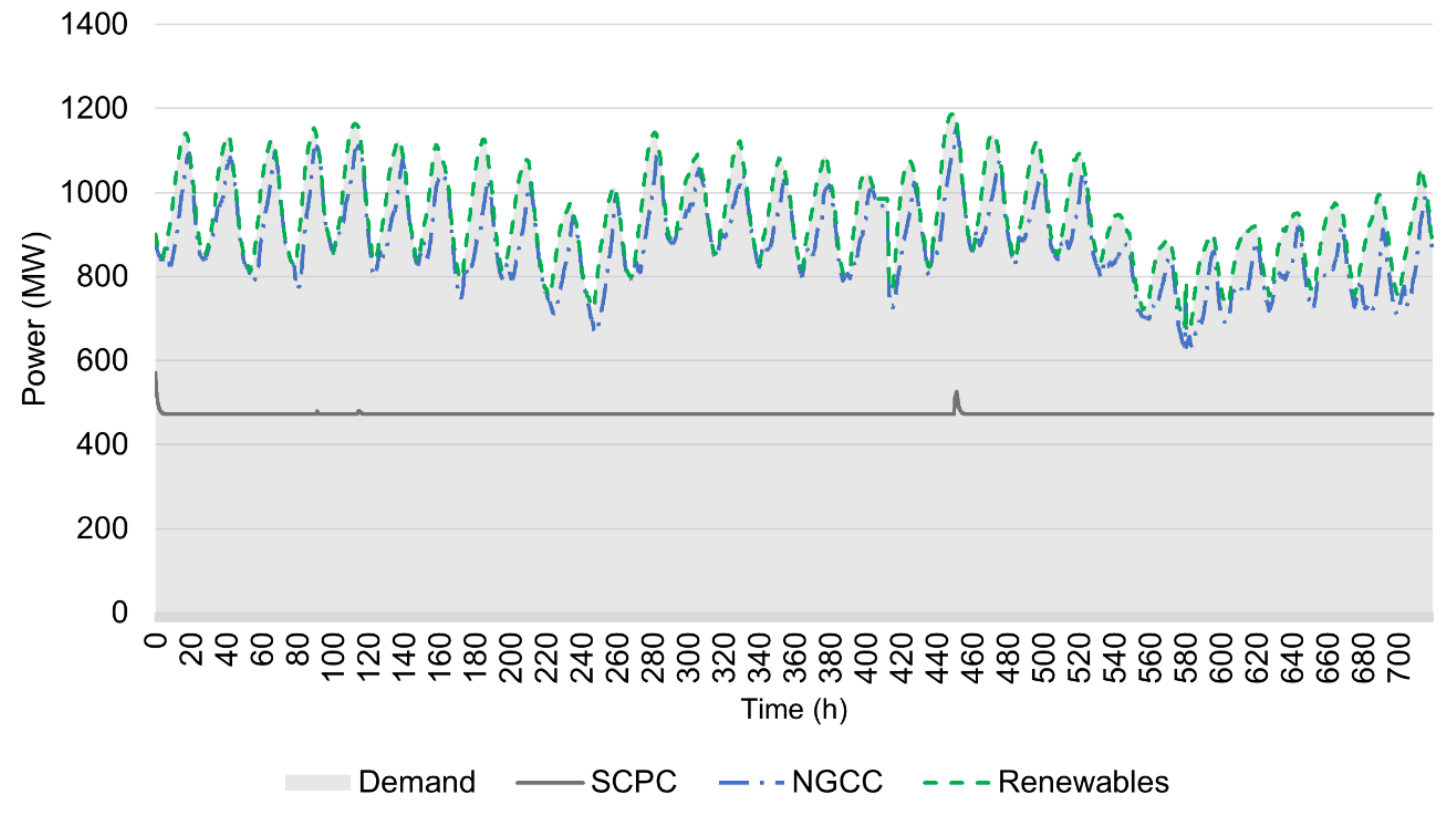

Figure 5.19. Power dispatch optimization result for Case 3

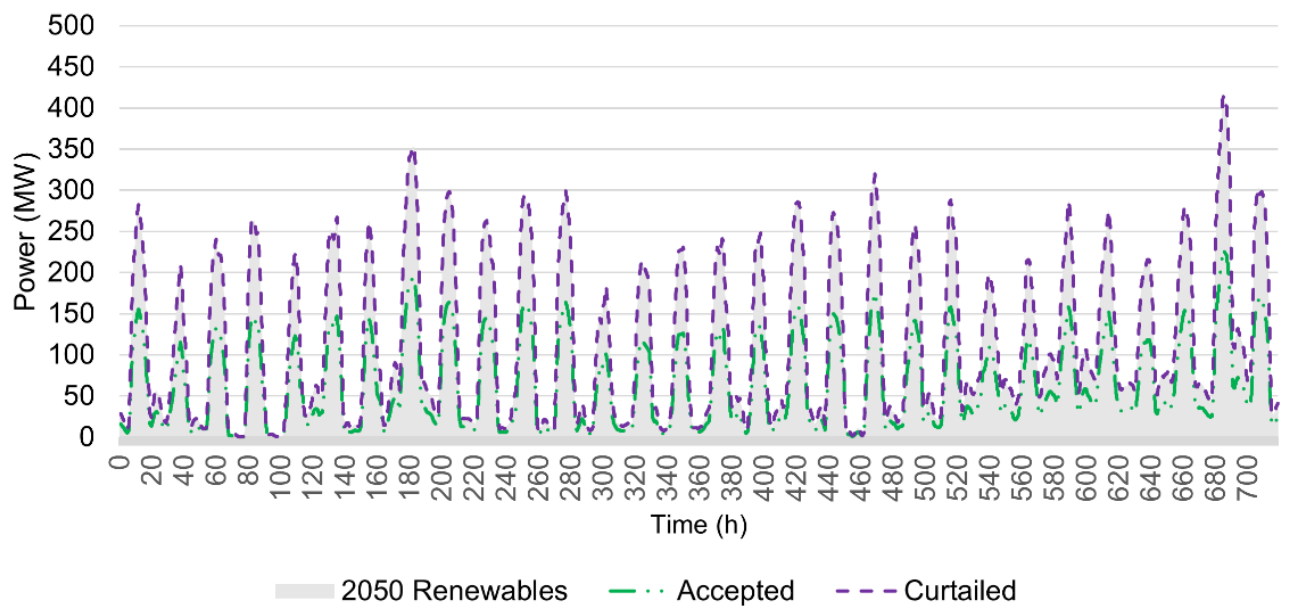

Figure 5.20. 2050 renewable levels accepted and curtailed for Case 3 


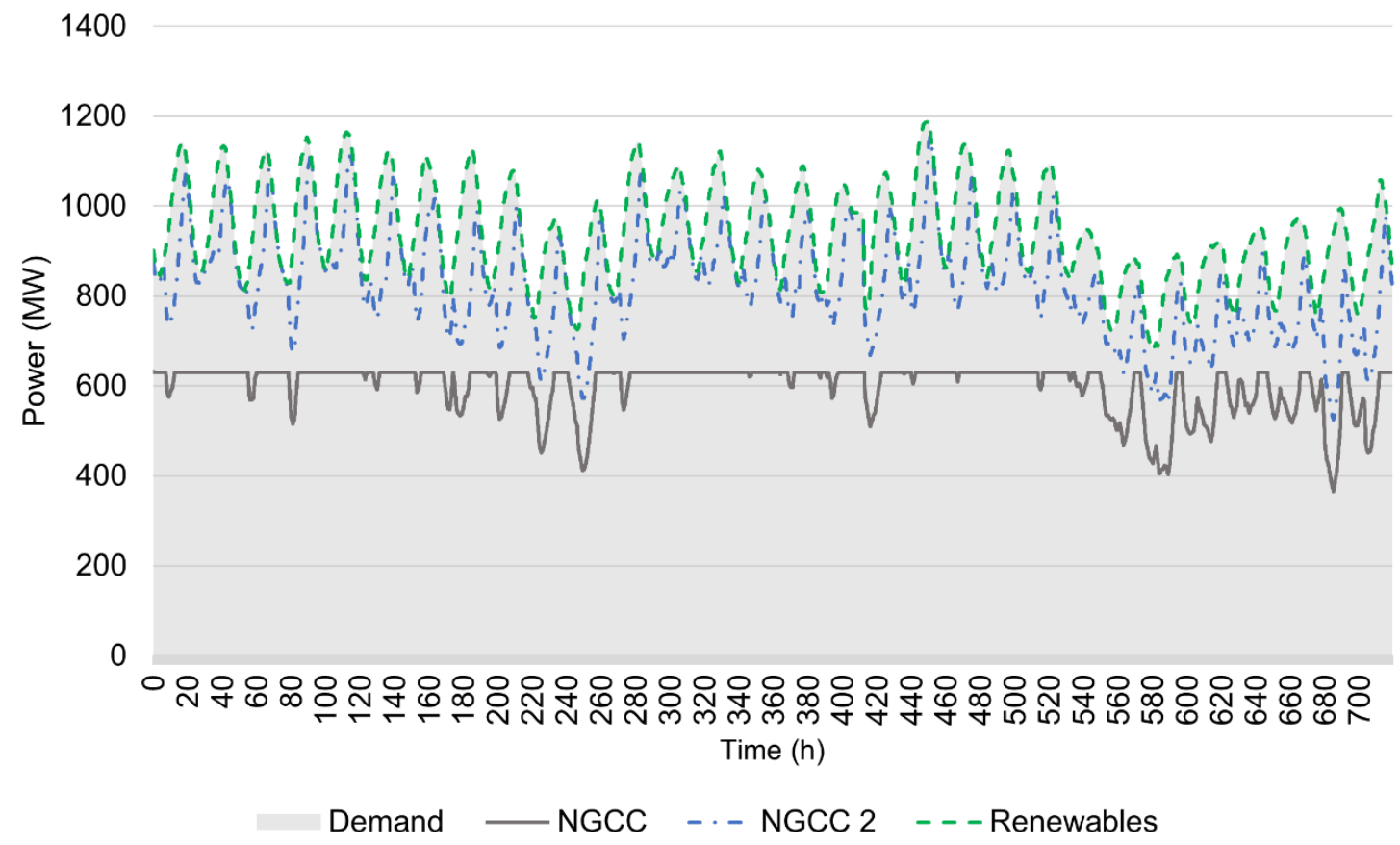

Figure 5.21. Power dispatch optimization result for Case 4

Case 1 considers the year 2019 for electricity price, demand, and solar/wind penetration into the grid. The power grid in this case is composed of SCPC, NGCC, and NaS batteries. The main reason for simulating Case 1 is to check the optimizer's ability to dispatch the energy systems considering the power demand without curtailment of renewables. Results depicted in Figure 5.17 show that for Case 1, the load demand is supplied by the SCPC and NGCC with no curtailment of the renewable energy sources while considering potential damage for the NGCC power plant. The NaS batteries are not utilized at any point. The results obtained are in agreement with the usual strategy employed nowadays, as there is no large-scale deployment of NaS batteries. Essentially, the results indicated that at the 2019 renewables penetration levels, the already installed SCPC and NGCC are sufficient to supply the energy within the acceptable planned damage maximum without requiring curtailment or usage of $\mathrm{NaS}$ batteries.

Case 2 considers a modification to the 2019 demand by increasing the minimum and maximum loads, i.e., the valleys and peaks, which would require faster ramping abilities from the grid. The electricity price and solar/wind penetrations into the grid are considered 
to be the same as in Case 1. The rationale in Case 2 is to verify the grid's capability to supply the demand under a more challenging demand peak/valley scenario. The results of this case are shown in Figure 5.18. In particular, the batteries power flow is plotted separately to facilitate the visualization. Figure 5.18 shows that the load demand is supplied mainly by the SCPC and NGCC, while the NaS batteries are used only twice (as highlighted in Figure 5.18 and plotted separately) to smooth the loads at times of $450 \mathrm{~h}$ and $580 \mathrm{~h}$. Specifically, at approximately $580 \mathrm{~h}$, the sum of energy provided by the SCPC, NGCC, and renewables (green dashed line) surpasses the total load demand (shaded area). The reason is that there is an excess of electricity in the grid, which is used to recharge the batteries and the power remains balanced (orange line matches with shaded area). Due to the battery operating costs, they are used only as a last option to prevent infeasibility, such as disruption of energy supply. As a perfect demand forecast of 6 min is assumed by the dispatcher, it is considered that any sudden deviation that was to happen could be supplied by costlier spinning reserves. The results obtained in Case 2 are in accordance with the literature, in which the batteries are often employed in the absence of transmission structure or isolated areas where energy sources are scarce (IRENA, 2015).

In Case 3, the electricity price, grid components, and demand are held constant and equal to those in Case 1, while the solar/wind penetrations are switched from 2019 to 2050 values. The 2050 wind/solar penetrations are shown in Figure 5.15. The 2050 intermittent renewable energy penetration is based on the 2019 penetration and Table 5.1 proportionality factors. The goal of simulating Case 3 is to verify the potential of 2019 grid components to supply the demand considering higher penetration of wind/solar sources. The results for Case 3 depicted in Figure 5.19 show that the load demand is supplied by the SCPC and NGCC components. However, to avoid oversupply of energy during dispatch, a $45 \%$ maximum curtailment is allowed. Figure 5.20 shows the curves for the accepted and curtailed renewable levels in Case 3. Under these circumstances, the batteries are not enough to avoid curtailment. To lower levels of curtailment, the optimizer stopped mostly after utilizing the energy available in the battery. This result indicates that considering the 2050 renewables penetration projection, the current grid is not sufficiently flexible to prevent renewable curtailment. The reasons may be because the power plants 
are not capable to ramp up/down fast enough, the minimum load of the power plant is too high, and the batteries do not have enough capacity available to manage the variability introduced by the renewables.

Finally, Case 4 considers the same forcing functions as in Case 3; however, the grid is revised by retiring the SCPC and including another 641 MWe NGCC. The goal of this case study is to assess how the change in grid components may impact renewable curtailment and grid flexibility. The results depicted in Figure 5.21 show that the demand load is supplied by the two NGCC power plants: one operated mostly as a baseload power plant and the other operated as a load-following power plant. This modification allowed the dispatch to achieve 0\% renewable curtailment levels for 2050 . This result indicates that for the grid to absorb higher variable renewable integration, there is a need to substantially increase grid flexibility. In Case 4 , the simulated scenario is to replace the SCPC by an NGCC, but other alternatives include retrofitting the SCPC, increasing NaS batteries capacity-albeit not being necessarily cost effective, or introducing an alternative energy storage system with higher capacity than the NaS batteries for a lower cost, such as pumped hydro, thermal energy storage, compressed air storage, and others.

Overall, it is observed that the SCPC is mostly dispatched at a constant power output while the NGCC is dispatched as load-following power plant when both technologies are available. This behavior is expected as the SCPC used in this work was designed as baseload and it is a less flexible technology than the NGCC. The average calculation time to determine the optimal dispatch for Case 1 to 4 was approximately $1200 \mathrm{~s}$.

Then, after the optimal dispatch was determined, $\mathrm{CO}_{2}$ equivalent and concentrated stress analyses are performed for each case study. The calculated $\mathrm{CO}_{2}$ equivalent is for the 30 day timespan and is depicted in Figure 5.22. 


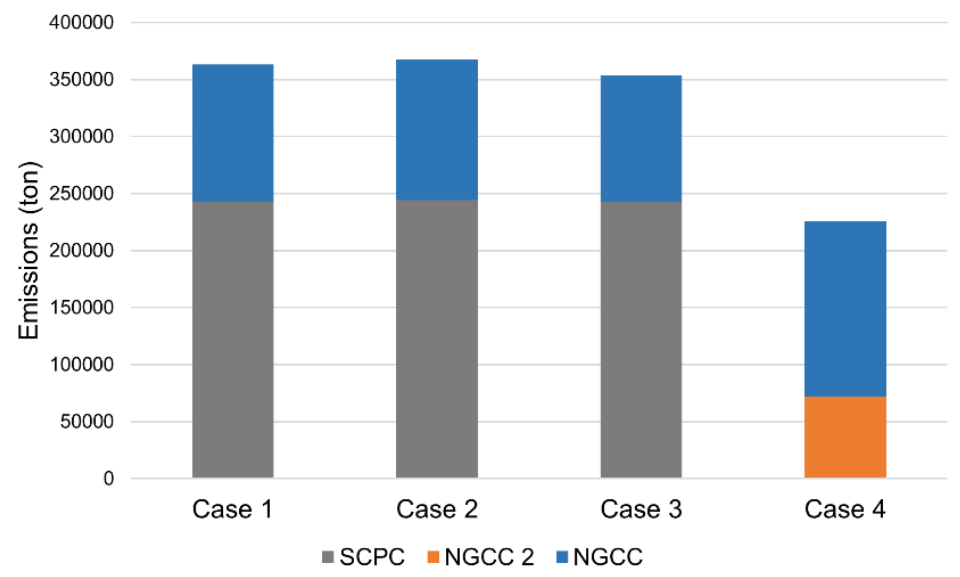

Figure 5.22. $\mathrm{CO}_{2}$ equivalent emissions by case study

In Figure 5.22, note that most of the $\mathrm{CO}_{2}$ emissions derive from the SCPC power plant, as the NGCC has a lower rate of $\mathrm{CO}_{2}$ emissions per generated unit of power. In particular, even though Case 3 has the 2050 renewable power generation, the amount of $\mathrm{CO}_{2}$ emissions is similar to that in Cases 1 and 2, as renewables curtailment was allowed in Case 3. As a remark, Cases 1 and 2 both had $0 \%$ curtailment of the 2019 renewables. Therefore, the results of Case 3 indicate that 2050 renewables were curtailed almost to the same levels as of 2019. This result implies that increasing the renewable production should be done along with increasing grid flexibility with cleaner and more efficient energy systems; otherwise, the environmental performance may not improve as expected when compared to the 2019 level of renewable penetration. A possibility to lower the emissions would be to combine the SCPC with a carbon capture technology. The carbon capture unit would have an energy penalty for the SCPC as mentioned in Chapter 4, but it would substantially decrease the $\mathrm{CO}_{2}$ emissions from SCPC.

Figure 5.23 shows both stress results on the body and at the most stressed part of the NGCC drum for all case studies. As Case 4 has two NGCCs in operation, stress analysis is performed to both power plants. 


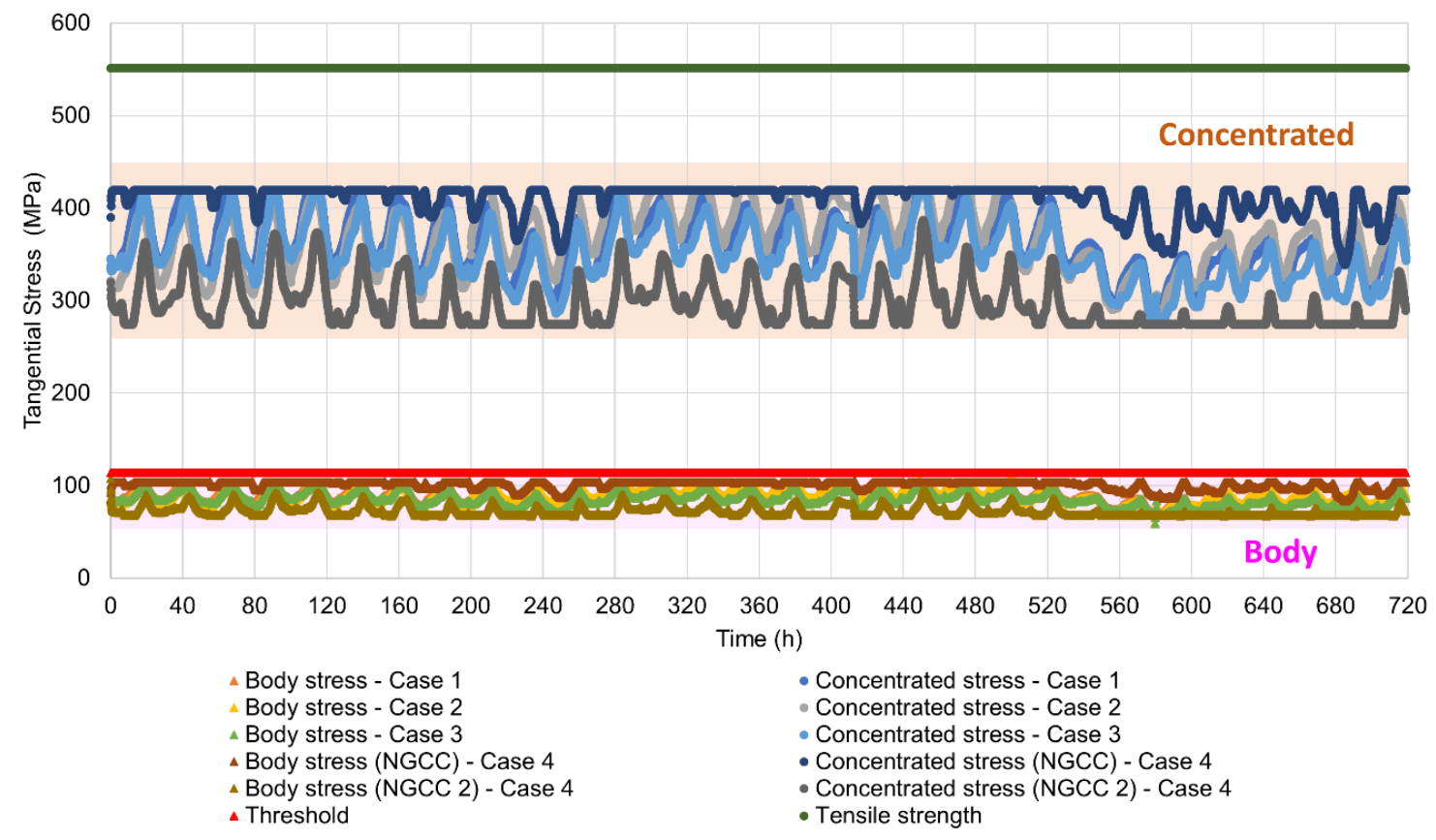

Figure 5.23. Drum body and concentrated tangential stresses during dispatch for Cases 1 to 4

In Figure 5.23, there are two shaded regions, namely "Body" (highlighted in pink in Figure 5.23) and "Concentrated" (highlighted in orange in Figure 5.23). The "Body" section refers to the overall stress that the body of the drum is subjected to, and the maximum value of this stress is considered as a constraint in the optimal dispatch algorithm. Although close to the threshold value, the optimal dispatch was determined without the body stress constraint ever becoming active. The "Concentrated" section in Figure 5.23 refers to the post-optimization stress analysis in the most stressed part of the drum. The stress in this specific part remained higher than the body stress threshold as well as the yield strength (260 MPa for the current MOC), but lower than the tensile strength. Thus, local uniform plastic deformation is expected to occur during a number of initial cycles in the most stressed part of the NGCC drum. However, the stress does not exceed the elastic shakedown limit, which is defined as twice the yield strength (520 MPa), and fully elastic behavior is anticipated in subsequent cycles.

Based on Case 4 results, Case 5 was elaborated under an even more challenging scenario regarding renewable penetration that demands up to $30 \% / \mathrm{min}$ ramping rates 
from the power plants and shorter time steps (36 sec). Therefore, a shorter overall time horizon load and renewable penetration curves are created and shown in Figures 5.24 and 5.25. The grid components are the same as Case 4, i.e., the two NGCCs and the NaS batteries.

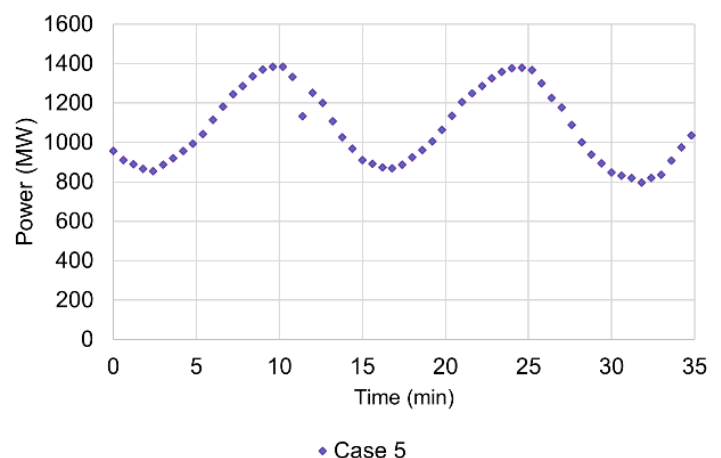

Figure 5.24. Modified electricity demand load for Case 5

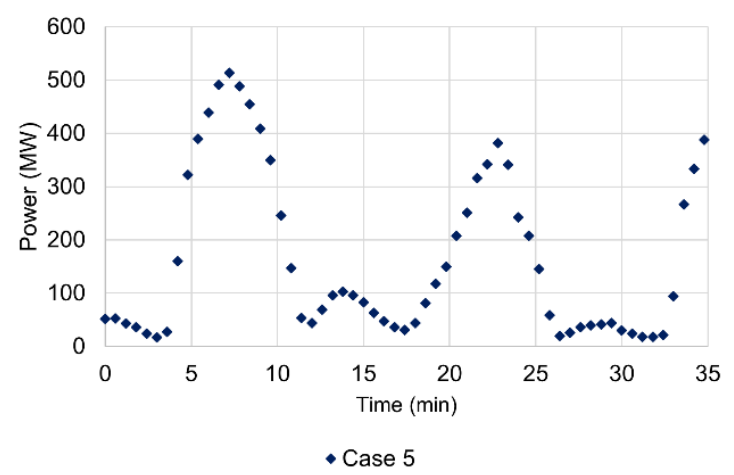

Figure 5.25. Modified variable renewable energy penetrations (solar/wind) for Case 5

The goal of this new case study is to give an insight about the NGCC drum thickness influence on the optimization objective function as well as the stresses on the NGCC's drums under high ramping rates scenario. Therefore, a sensitivity study is conducted initially considering a range of $[0.1461 \mathrm{~m}, 0.1905 \mathrm{~m}]$ for both drums' thicknesses (from NGCC and NGCC 2), while holding all other forcing functions constant. The results of the sensitivity study are shown in Figure 5.26. The results indicate that for cases in which the cycling leads to prohibitive stresses, the optimization objective showed some sensitivity to the drum thickness. It is expected for the objective to become more sensitive as the number of NGCC's considered in the grid increases. It was also observed that the thicknesses are not the same for baseload and load-following power plants to achieve the strict minimum value in the sensitivity analysis. At drum thicknesses higher than 0.1905 $\mathrm{m}$, the dispatch was not completed, as prohibitive stresses reduced the feasible space to null at certain time instances. 


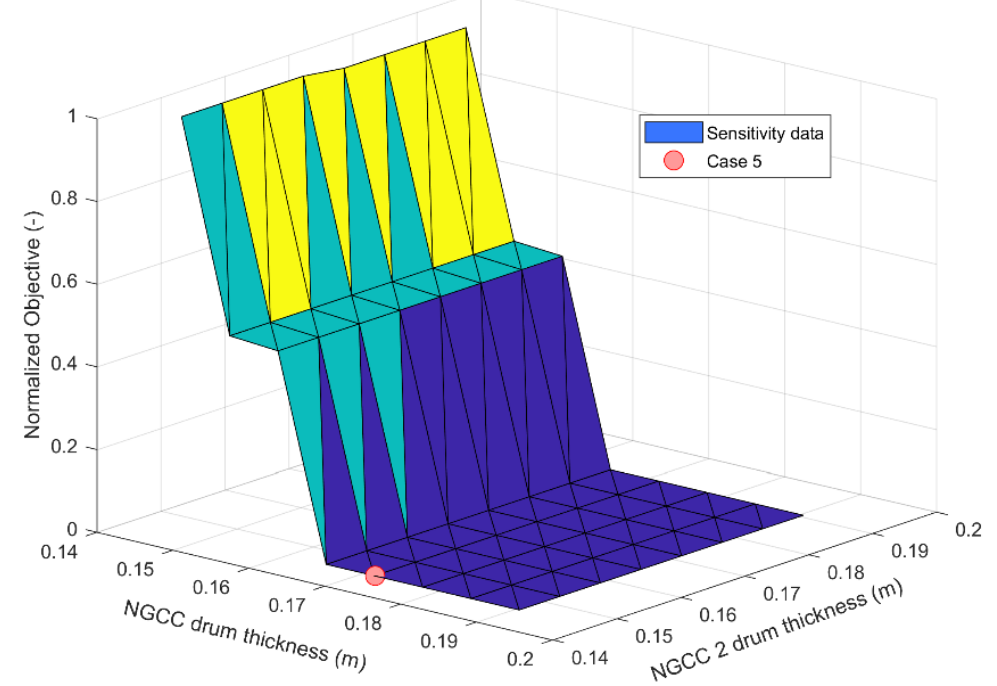

Figure 5.26. Sensitivity analysis of optimization objective due to NGCC and NGCC 2 drums' thicknesses

After the sensitivity analysis was completed, a point for the normalized objective equal to zero is chosen for the optimal dispatch simulation. The drum thickness for the NGCC is $0.17145 \mathrm{~m}$ and for the NGCC 2 , it is equal to $0.1461 \mathrm{~m}$. The results of the optimal dispatch and corresponding stresses are shown in Figures 5.27 and 5.28. Figure 5.27 shows that under a highly variable renewables scenario that demands faster ramping rates, the $\mathrm{NaS}$ batteries are used more often, discharging at approximately $t=9.6 \mathrm{~min}$ and $t=24 \mathrm{~min}$. These results indicate that under more aggressive ramp rates $(>30 \% / \mathrm{min})$, the use of batteries could have a larger participation in the grid. Curtailment is still avoided under this scenario, while NGCC acts as baseload power plant, ramping down when there is excess of renewable energy, and NGCC 2 acts as load following power plant, cycling its load more often than the other NGCC. Figure 5.28 shows that the drum body stress constraint was active at times for NGCC 2, while for the other NGCC, the stress value was close but below the threshold. In addition, as the threshold is dependent on drum thickness, the thresholds for NGCC and NGCC 2 are slightly different. 


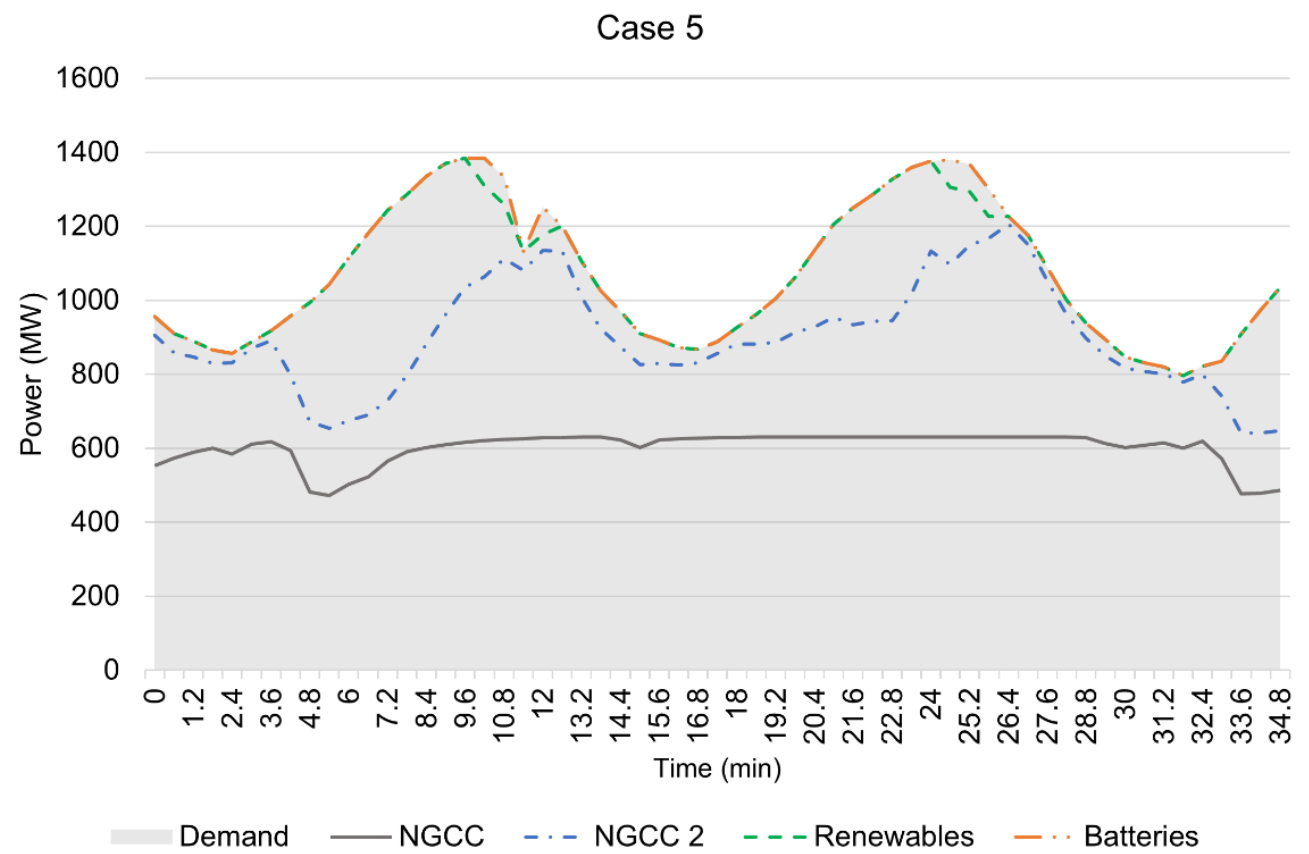

Figure 5.27. Power dispatch optimization result for Case 5

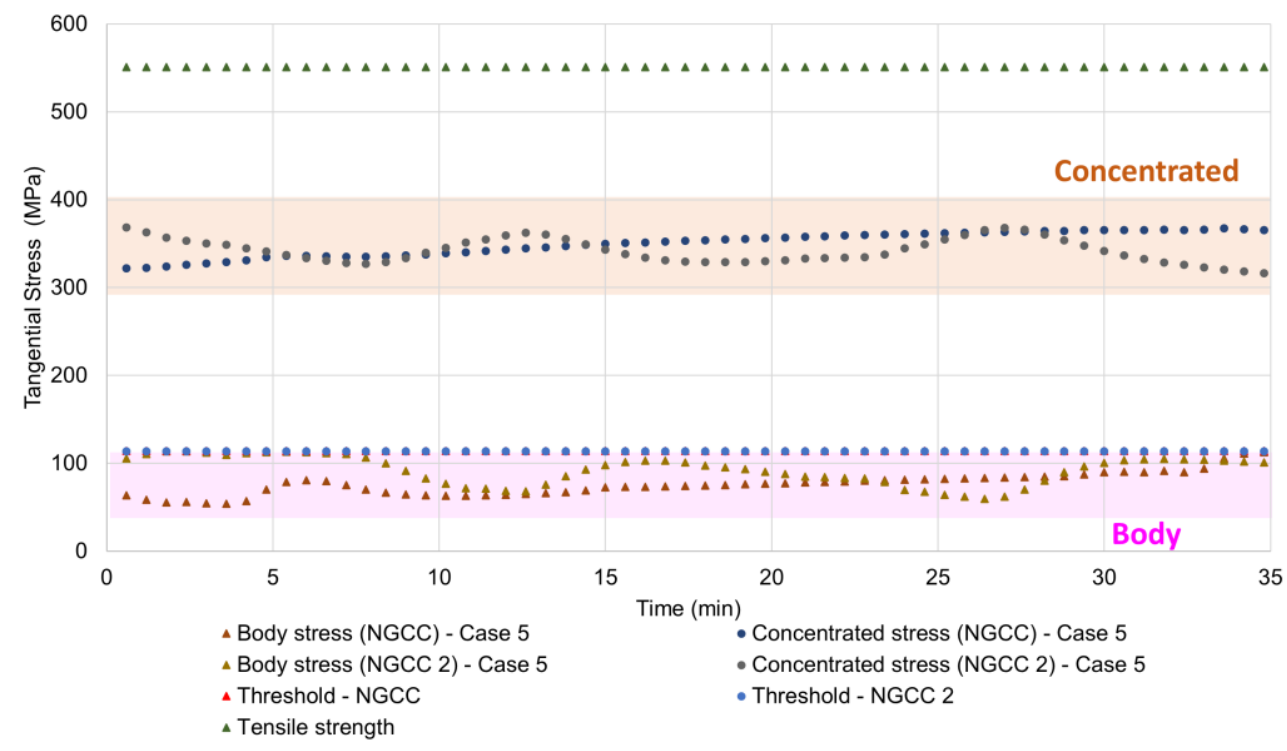

Figure 5.28. Drum body and concentrated tangential stresses during dispatch for Case 5

Further analysis is performed considering the individual components of the body stress depicted in Figures 5.29 and 5.30. For the NGCC, the mechanical stress, which is tensile 
in nature, was mostly at its design point, with the thermal stress frequently being compressive and relieving the overall body stress. This behavior agrees with the NGCC dispatch, which is employed often as baseload with minimal cycling. For the NGCC 2, the mechanical stress was often lower than its design point, which allowed a larger margin for thermal stress to vary between tensile and compressive stresses. This behavior agrees with the NGCC 2 dispatch, which is employed often as load-following and with more frequent cycling. This difference in mechanical and thermal stresses behavior between baseload and load-following NGCC power plants reinforces the need to include a equipment health-related constraint when cycling is substantial.

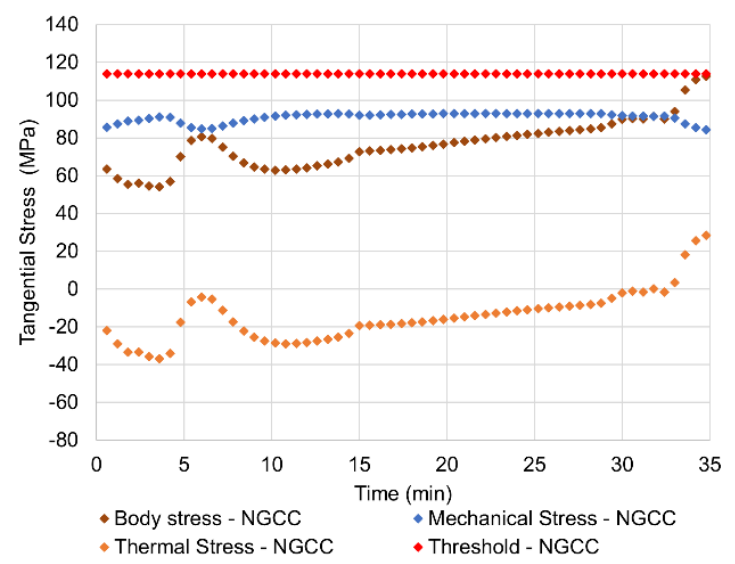

Figure 5.29. Mechanical and thermal stresses for NGCC for Case 5

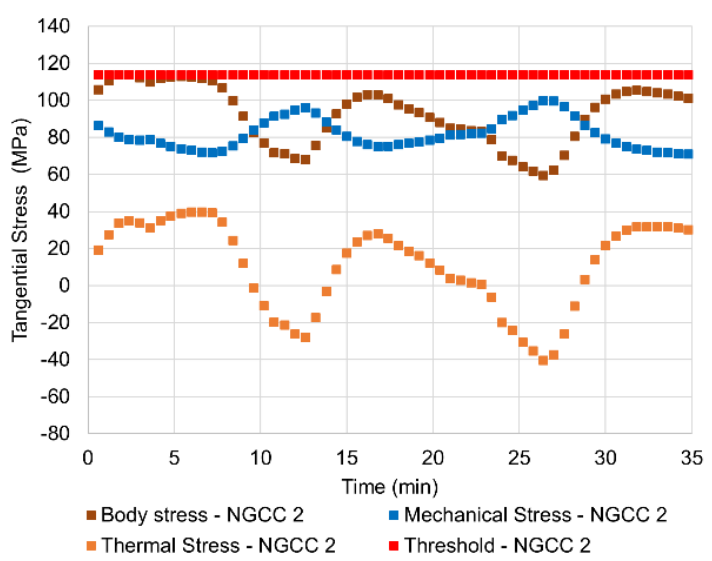

Figure 5.30. Mechanical and thermal stresses for NGCC 2 for Case 5 


\section{Conclusions}

In this dissertation, dynamic optimization algorithms were developed for optimal operations and dispatch of cycling baseload energy systems under VRE penetration. Particularly, two different dynamic optimization strategies were established and implemented to fill the identified gaps: the nonlinear multi-objective dynamic real-time predictive optimization (NMPO) and the mixed-integer linear programming (MILP) formulation for dynamic dispatch of energy systems while considering the power plant health.

The hourly-level strategy is a NMPO and it was successfully implemented in a supercritical pulverized coal-fired (SCPC) power plant with a postcombustion carbon capture system (CCS), considering economic and environmental objectives. Different strategies were employed and explored to improve computational tractability, such as mathematical reformulations, automatic differentiation (AD), and parallelization of a metaheuristic particle swarm optimization (PSO) component. The parallelization of the PSO in a single desktop improved the computational time in 4-folds when compared to the traditional PSO. The AD further improved the computational time within the range of 80 to 120 -folds when compared to a default finite-differences method.

The minute-level strategy is based on a mixed-integer linear programming (MILP) formulation for the dynamic dispatch of energy systems while considering equipment health-related constraints. This MILP framework was successfully implemented in a suite of systems that includes natural gas-fired power plant, coal-fired power plant, and sodium sulfur batteries in addition to the VRE systems. Five case studies were simulated considering different loads and renewable penetration levels. In general, the results showed that grid flexibility was mostly provided by the natural gas power plant, while the batteries were used sparingly. Additionally, considering the post-optimization equivalent carbon analysis, the environmental performance was intrinsically connected to grid flexibility and the level of VRE penetration. The stress results reinforced the necessity of further considering and including equipment health-related constraints during dispatch, as power plant design and stress behavior were distinct if the power plant acted either as at constant power output or load-following operations. 
The results of the NMPO successfully implemented for a large-scale SCPC-CCS showed the benefit of having an optimization horizon longer than the implementation horizon. In particular, a prediction capability allowed the algorithm to set up optimal conditions ahead of time to reach lower costs while maintaining the environmental performance. Results also showed that the optimal compromise from the Pareto front was chosen according to a set of weights for the objectives with minimal interaction between the framework and the decision maker. The results of the modified Tchebycheff-based multi-objective component demonstrated that the optimal trajectories were selected automatically based on the decision maker weight vector and remained in balance between both objectives at all times. The $\epsilon$-constraint method was also briefly explored in this dissertation and it was concluded that this method would be more suitable to strategies that do not have a rolling horizon feature, such as scheduling and unit commitment problems. Finally, the market and carbon policies had impact on the optimal compromise between the economic and environmental objectives. When considering carbon capture economic incentives, results showed an increase of approximately 63 ton of $\mathrm{CO}_{2}$ captured over $12 \mathrm{~h}$ operation for the optimal compromise when compared to an unfavorable market condition.

In conclusion, the developed dynamic optimization algorithms for baseload power plant cycling under VRE were successfully developed and implemented for the minute- and hourly-basis time scale of power system operation. The algorithms encompassed strategies to improve computational time, power plant health-related constraints, and power systems dynamic information for power plant optimal performance under cycling. The results also indicated a path forward to include non-economic concepts during operations optimization, which can lead to higher preparedness and resilience of the grid. 


\section{Future Recommendations}

\subsection{Coding Environment}

In this dissertation, the NMPO and MILP-based dynamic dispatch algorithms and models are built in MATLAB, which constrained the choice of solvers and parallelization structures. To overcome these challenges, free third party software packages are used such as the ADiMAT and OPTI toolbox and the ParPSO and PSO were recreated and adapted for MATLAB environment. Another option that should be explored is to investigate Python programming language due to its multi-threading friendly, objectoriented programming, and extensive open-source libraries.

\subsection{Optimization Formulation and Connection with MPC-based Controller}

Currently, most of the employed surrogate dynamic models are discretized linear and nonlinear ARX models and the NMPO was applied only to two objectives. In the future, a third or more objectives such as power plant safety could be included in the NMPO structure. The varying operating temperature and pressure motivate the implementation of an equipment stress-based constraint that considers the pipeline age and material of construction. The increase of computational time could be addressed by using other surrogate models such as Gaussian process models and D-RM® in conjunction with Python programming. Also, the current framework is limited by the discretized domain and fixed time step. A ROM in the continuous domain should be explored for the presented optimization frameworks. Finally, the trajectories generated in this dissertation are open-loop trajectories. Therefore, for real world applications, it is recommended pairing the NMPO with an MPC-based controller to close the loop.

7.3. Unit Commitment Problem with Equipment Health-related Constraints and Nonlinear Programming

In this dissertation, a MILP-based optimization framework is developed to dispatch energy systems under VRE while considering power plant health. The linear programming introduced inherent inaccuracies to the high-fidelity models, which a nonlinear programming framework should be able to address. Inclusion of fatigue-based damage models during unit commitment (UC) problem should also be explored as the complete 
cycle damage could be accounted during scheduling. Furthermore, energy reserve management and grid resilience scenarios should be included while solving a UC problem. At last, a $\epsilon$-constrained UC-MOO formulation should be further investigated as this $\mathrm{MOO}$ method is ideal for a non-rolling horizon implementation and requires a lower number of optimization evaluations when compared to the employed Modified Tchebycheff MOO method.

\subsection{Dynamic Stochastic Optimization}

In this dissertation, all the optimizations are deterministic. However, the VRE, power demands, and electricity prices have intrinsic variability and often their predictions vary from the realized values. Thus, one way to address this challenge would be to include the stochasticity of these functions in the optimizer. This strategy would increase the computational time and it should be addressed by either employing similar approaches used in this work or the suggestions mentioned in items 7.1 and 7.2. 


\section{References}

Abouhawwash, M., Jameel, M., Deb, K., 2020. A Smooth Proximity Measure for Optimality in Multi-objective Optimization using Benson's Method. Computers and Operations Research, 117. doi:10.1016/j.cor.2020.104900.

Angeli, D., Amrit, R., Rawlings, J. B., 2012. On Average Performance and Stability of Economic Model Predictive Control. IEEE Transactions on Automatic Control, 57(7):1615-1626. doi: 10.1109/TAC.2011.2179349.

Bankole, T., Jones, D., Bhattacharyya, D., Turton, R., Zitney, S. E., 2018. Optimal Scheduling and Its Lyapunov Stability for Advanced Load-Following Energy Plants with $\mathrm{CO}_{2}$ Capture. Computers and Chemical Engineering, 109:30-47. doi: 10.1016/j.compchemeng.2017.10.025.

Bentek Energy LLC, 2010. How less became more.... Wind power, and unintended consequences in the Colorado energy market. https://www.westernenergyalliance.org/wp-

content/uploads/BENTE_IPAMS_Wind_Study.pdf (accessed on 01.30.2019).

Biegler, L.T., 2009. Technology Advances for Dynamic Real-Time Optimization. Proceeding of 10th International Symposium on Process System Engineering PSE 2009, August, Bahia, Brazil 2009.

Bischof, C.H., Bücker, H.M., Lang, B., Rasch, A., Vehreschild, A., 2002. Combining Source Transformation and Operator Overloading Techniques to Compute Derivatives for MATLAB Programs. Proceedings of the Second IEEE International Workshop on Source Code Analysis and Manipulation, Montreal, QC, Canada, 6572, doi: 10.1109/SCAM.2002.1134106.

Câmara, M. M., Quelhas, A. D., Pinto, J. C., 2016. Performance Evaluation of Real Industrial RTO Systems. Processes, 4(4):44. doi: 10.3390/pr4040044.

Carrasco, J.C., Lima, F.V., 2018. Bilevel and Parallel Programing-based Operability Approaches for Process Intensification and Modularity. AIChE Journal, 64(8):3042-3054. doi:10.1002/aic.16113. 
CEP, 2021. Chemical Engineering Magazine. Chemical Engineering Plant Cost Index. www.chemengonline.com/pci (accessed on 02.06.2021).

CCSI, 2018. D-RM Builder - User Manual version 2.0.0. March 2018. https://github.com/CCSI-Toolset/drmbuilder/tree/master/docs (accessed on 02.25.2021).

Coello, C. A. C, Lamont, G. B., Van Veldhuisen, D. A., 2007. Evolutionary algorithms for solving multi-objective problems. 2nd edn. New York: Springer (Genetic and evolutionary computation series).

CTC, 2021. Carbon Tax Center: Where Caron is Taxed. www.carbontax.org/wherecarbon-is-taxed/. (accessed on 02.06.2021).

Currie, J., Wilson, D.I., 2012. OPTI: Lowering the Barrier Between Open Source Optimizers and the Industrial MATLAB User. Proceeding of Foundations of Computer-Aided Process Operations, FOCAPO/CPC, Georgia, USA.

Diwekar U. M., 2008. Introduction to Applied Optimization. 2nd edn. New York: Springer (Springer Optimization and its Applications, v. 22).

Dowell, N. M., Shah, N.,2014. Optimisation of Post-Combustion $\mathrm{CO}_{2}$ Capture for Flexible Operation. Energy Procedia, 63:1525-1535.

EIA, 2019a. Annual Energy Outlook 2019: With Projections to 2050, https://www.eia.gov/outlooks/aeo/pdf/AEO2019.pdf (accessed on 02.02.2021).

EIA, 2019b. Energy Information Administration. Coal - Recent Data. https://www.eia.gov/coal/. (accessed on 06.01.2020).

EIA, 2019c. US Natural Gas Prices. https://www.eia.gov/dnav/ng/ng_pri_sum_dcu_nus_m.htm (accessed on 06.01.19)

EIA, 2021. Energy Information Administration. Coal - Recent Data. https://www.eia.gov/coal/. (accessed on 02.13.2021).

Ela, E., Kemper, J., 2009. Wind Plant Ramping Behavior. December 2009. NREL/TP550-46938. https://www.nrel.gov/docs/fy10osti/46938.pdf (accessed on 02.15.2021). 
Elaiw, A. M., Xia, X., Shehata, A. M., 2013. Hybrid DE-SQP and hybrid PSO-SQP methods for solving dynamic economic emission dispatch problem with valvepoints effects. Electric Power Systems Research, 103:192-200.

ERCOT, 2021a. Grid operator requests energy conservation for system reliability. $\begin{array}{llll}\text { Published on } & \text { February } & 14^{\text {th }} \text {, } & 2021 .\end{array}$ http://www.ercot.com/news/releases/show/225151 (accessed on 02.17.2021).

ERCOT, 2021b. ERCOT calls for rotating outages as extreme winter weather forces generating units offline. Published on February $15^{\text {th }}, 2021$. http://www.ercot.com/news/releases/show/225210 (accessed on 02.17.2021).

European Committee for Standardization, 2002. EN 13345 Part 3, Unfired Pressure Vessels, Clause 17; Simplified Assessment of Fatigue Life, and Clause 18; Detailed Assessment of Latigue https://www.unm.fr/medias/files/107_FICHIER_0.pdf (accessed on 05.23.2020)

FERC, 2011. Outages and Curtailments During the Southwest Cold Weather Event of February 1-5, 2011. Causes and Recommendations. Prepared by the staff of the Federal Energy Regulatory Commission and the North American Electric Reliability Corporation. August 2011. https://www.ferc.gov/sites/default/files/202004/08-16-11-report.pdf (accessed on 02.17.2021).

FUTURE Act, 2017. S.1535 Bill - 115th Congress (2017-2018). https://www.congress.gov/bill/115th-congress/senate-bill/1535/text. (accessed on 03.09.2021).

Grol, E., Tarka, T. J., Myles, P., Bartone, Jr., Leonard, M., Simpson J., Rossi, G., 2015. Impact of Load Following on the Economics of Existing Coal-Fired Power Plant Operations. June 3, 2015. DOE/NETL-2015/1718. Web. doi:10.2172/1513827.

He, X., Lima, F.V., 2020. A Modified SQP-based Model Predictive Control Algorithm: Application to Supercritical Coal-fired Power Plant Cycling. Industrial and Engineering Chemistry Research, 59(35):15671-15681. doi:10.1021/acs.iecr.0c01843. 
Henan Steel Guang International Trade Co, LTD, 2020. http://www.steelplatecuttingpart.com/products/asme-sa515-boiler-or-pressurevessel-steel-plate.html (accessed on 04.01.2020).

Houser, T., Bordoff, J., Marsters, P., 2017. Can Coal Make a Comeback. Columbia SIPA, Center on Global Energy Policy.

Intergovernmental Panel on Climate Change - IPCC. (2018). Summary for Policymakers. In: Global warming of $1.5^{\circ} \mathrm{C}$. An IPCC Special Report on the impacts of global warming of $1.5^{\circ} \mathrm{C}$ above pre-industrial levels and related global greenhouse gas emission pathways, in the context of strengthening the global response to the threat of climate change, sustainable development, and efforts to eradicate poverty [V. Masson-Delmotte, P. Zhai, H. O. Pörtner, D. Roberts, J. Skea, P.R. Shukla, A. Pirani, W. Moufouma-Okia, C. Péan, R. Pidcock, S. Connors, J. B. R. Matthews, Y. Chen, X. Zhou, M. I. Gomis, E. Lonnoy, T. Maycock, M. Tignor, T. Waterfield (eds.)].

IRENA, 2015. Battery Storage for Renewables: Market Status and Technology Outlook. January 2015. https://www.irena.org/documentdownloads/publications/irena_battery_storage_re port_2015.pdf (accessed on 12.12.2019).

Jamaludin, M. Z, Swartz C. L. E., 2017. Dynamic Real-Time Optimization with ClosedLoop Prediction. AIChE Journal, 63(9):3896-3911.

Kim, R., Wang, Y., Vudata, S. P., Bhattacharyya, D., Lima, F. V., and Turton, R., 2020. Dynamic Optimal Dispatch of Energy Systems with Intermittent Renewables and Damage Model. Mathematics 8(868). doi:10.3390/math8060868.

Kim, T. S., Lee, D.K., Ro, S.T., 2010. Analysis of thermal stress evolution in the steam drum during start-up of a heat recovery steam generator. Applied Thermal Engineering, 20:977-992.

Ljung L., 2021. System Identification Toolbox ${ }^{\mathrm{TM}}$. User's Guide. Revised on March 2021. https://www.mathworks.com/help/pdf_doc/ident/ident_ug.pdf. (accessed on 04.09.2021) 
Li, H., Swartz, C. L. E., 2018. Approximation Techniques for Dynamic Real-time Optimization (DRTO) of Distributed MPC Systems. Computers and Chemical Engineering, 118:195-209. doi:10.1016/j.compchemeng.2018.08.002.

Ma, J., Mahapatra, P., Zitney, S. E., Biegler, L. T., \& Miller, D. C., 2016. D-RM builder: a software tool for generating fast and accurate nonlinear dynamic reduced models from high-fidelity models. Computers and Chemical Engineering, 94:60-74. https://doi.org/10.1016/j.compchemeng.2016.07.021.

Ma, X., Zhang, Q., Tian, G., Yang, J., Zhu, Z., 2018. On Tchebycheff Decomposition Approaches for Multiobjective Evolutionary Optimization. IEEE Transactions on Evolutionary Computation, 22:226-244.

Mathworks, 2021.

Wavenet

Documentation. https://www.mathworks.com/help/ident/ref/wavenet.html (accessed on 02.24.2021)

McKubre, M. C. H., Tanzella, F. L., Smedley, S. I., 1989. The Electromotive Force of the $\mathrm{Na} / \mathrm{S}$ Cell. Journal of Electrochemical Society, 136:303-305, doi:10.1149/1.2096625.

Morales-España, G., Gentile, C., Ramos, A., 2015. Tight MIP formulations of the powerbased unit commitment problem. OR Spectrum, 37:929-950. doi: 10.1007/s00291015-0400-4.

Narzisi, G., 2008. Classic Methods for Multi-objective Optimization. Courant Institute of Mathematical Sciences, New York University. 31 January 2008.

NERC, 2020. North American Electric Reliability Corporation. 2020 State of Reliability. An Assessment of 2019 Bulk Power System Performance. July 2020. https://www.nerc.com/pa/RAPA/PA/Performance\%20Analysis\%20DL/NERC_SO R_2020.pdf (accessed on 03.01.2021).

NETL, 2015. Cost and Performance for Fossil Energy Plants. Volume 1a: Bituminous Coal (PC) and Natural Gas to Electricity. Revision 3. July 6, 2015. DOE/NETL2015/1723.

NETL, 2019. Cost and Performance Baseline for Fossil Energy Plants Volume 1: Bituminous Coal and Natural Gas to Electricity, NETL-PUB-22638. 
https://netl.doe.gov/projects/files/CostAndPerformanceBaselineForFossilEnergyP lantsVol1BitumCoalAndNGtoElectBBRRev4-1_092419.pdf. (accessed on 04.08.2020).

Oyama, H., Durand, H., 2020. Interactions Between Control and Process Design under Economic Model Predictive Control. Journal of Process Control, 92:1-18. doi:10.1016/j.jprocont.2020.05.009.

Pang, M., Shi, Y., Wang, W., Pang, S., 2019. Optimal sizing and control of hybrid energy storage systems for wind power using hybrid Parallel PSO-GA algorithm. Energy Exploration \& Exploitation, 37(1):558-578).

Pattison, R. C., Touretzky, C. R., Johansson, T., Harjunkoski, I., Baldea, M., 2016. Optimal Process Operations in Fast-Changing Electricity Markets: Framework for Scheduling with Low-Order Dynamic Models and an Air Separation Application. Industrial and Engineering Chemistry Research, 55:4562-4584 doi: 10.1021/acs.iecr.5b03499.

PJM, 2019. Markets \& Operations. Available online: https://www.pjm.com/markets-andoperations.aspx (accessed on 12.16.2019).

Pontes, K. V., Embirucu, M., Wolf, I. J., Marquardt, W., 2015. Dynamic Real-time Optimization of Industrial Polymerization Processes with Fast Dynamics. Industrial and Engineering Chemistry Research, 54(47):11881-11893. doi:10.1021/acs.iecr.5b00909.

REN21, 2018. Renewable Energy Policy Network for $21^{\text {st }}$ Century. http://www.ren21.net/status-of-renewables/global-status-report/ (accessed on 04.16.2018).

Rubin, E. S., Davison, J. E., Herzog, H. J., 2015. The Cost of $\mathrm{CO}_{2}$ Capture and Storage. International Journal of Greenhouse Gas Control, 40:378-400. doi: 10.1016/j.ijggc.2015.05.018.

Schaefer, S.; Vudata, S. P.; Bhattacharyya, D.; Turton, R., 2020. Transient modeling and simulation of a nonisothermal sodium-sulfur cell. Journal Power Sources, 453. doi: 10.1016/j.jpowsour.2020.227849. 
Schill, W., Pahle, M., Gambardella, C., 2017. Start-up costs of thermal power plants in markets with increasing shares of variable renewable generation. Supplementary Information. Nature Energy, 2, doi:10.1038/nenergy.2017.50.

Sengupta, S., Basak, S., Peter II, R.A., 2018. Particle Swarm Optimization: A survey of historical and recent developments with hybridization perspectives. Cornell University Library Arxiv, Neural and Evolutionary Computing. https://arxiv.org/abs/1804.05319. (accessed on 12.2018).

Sharafi, M., EIMekkawy, T., 2014. A dynamic MOPSO algorithms for multiobjective optimal design of hybrid renewable energy systems. International Journal of Energy Research, 38:1949-1963.

Sola M.C., 2010. Parallel Processing for Dynamic Multi-objective Optimization. Dissertation for the Degree of Doctor of Philosophy. Universidad de Granada. https://www.researchgate.net/profile/Julio_Ortega/publication/220951202_Paralle I_Processing_for_Multi-

objective_Optimization_in_Dynamic_Environments/links/Ofcfd50929ab23eb7900 0000.pdf (accessed on 02.02.2021).

Technical Rules for Steam Boilers, 1996. TRD 301, Annex 1, Design. Calculation for Cyclic Loading Due to Pulsating Internal Pressure or Combined Changes of Internal Pressure and Temperature. https://kupdf.net/download/trd-301-annex-1design_58c5ef24dc0d609909339028_pdf (accessed on 05.23.2020)

Thierry, D., Biegler, L.T., 2019. Dynamic Real-time Optimization for a $\mathrm{CO}_{2}$ Capture Process. AIChE Journal, 65(7).

Tosukhowong, T., Lee, J.M., Lee, J.H., Lu, J., 2004. An Introduction to a Dynamic Plantwide Optimization Strategy for an Integrated Plant. Computers and Chemical Engineering, 29(1):199-208. doi:10.1016/j.compchemeng.2004.07.028.

Tran, T., Ling, K., Maciejowski, J., 2014. Economic Model Predictive Control - A Review. Proceeding of 31st International Symposium on Automation and Robotics in Construction and Mining, ISARC. doi: 10.22260/ISARC2014/0006. 
Trifkovic, M., Marvin, W. A., Daoutidis, P., Sheikhzadeh, M., 2014. Dynamic Real-time Optimization and Control of a Hybrid Energy System. AIChE Journal, 60(7):25462556. doi:10.1002/aic.14458.

US DOE, 2017. Quadrennial Energy Review. Transforming the Nation's Electricity System: The Second Installment of the QER. January 2017. https://www.energy.gov/sites/prod/files/2017/02/ł34/Quadrennial\%20Energy\%20 Review--Second\%20Installment\%20\%28Full\%20Report\%29.pdf (accessed on 02.02.2021).

USAID-NREL Partnership, 2019. Greening the Grid. Demand response and storage. http://www.greeningthegrid.org/integration-in-depth/demand-response-andstorage (accessed on 04.07.2019).

Venter, G., Sobieszczanski-Sobieski, J., 2005. A Parallel Particle Swarm Optimization Algorithm Accelerated by Asynchronous Evaluations. Proceeding of $6^{\text {th }}$ World Congresses of Structural and Multidisciplinary Optimization, May, Rio de Janeiro, Brazil, 2005.

Vigerske, S., Wächter, A., 2021. Ipopt Documentation. https://coinor.github.io/lpopt/index.html\#Overview (accessed on 03.03.2021).

Wächter, A., Biegler, L.T., 2006. On the Implementation of a Primal-dual Interior Point Filter Line Search Algorithm for Large-scale Nonlinear Programming. Mathematical Programming, 106(1):25-57. Preprint at http://www.optimizationonline.org/DB_HTML/2004/03/836.html.

Wang, Y., Bhattacharyya, D., Turton, R., 2020. Evaluation of Novel Configurations of Natural Gas Combined Cycle (NGCC) Power Plants for Load-Following Operation using Dynamic Modeling and Optimization. Energy \& Fuels 34(1):1053-1070. doi: 10.1021/acs.energyfuels.9b03036.

Weber, P. J., Bleed, J., 2021. Power outages linger for millions as another icy storm looms. Published on February 17th, 2021. https://apnews.com/article/100-millionamericans-more-ice-snow-404012f8d8885259968b8d37b13899d6 (accessed on 02.17.2021). 
Wei, H. L., Billings, S. A., Balikhin, M. A., 2003. Wavelet Based Nonparametric NARX Models for Nonlinear Input-Output System Identification. Research Report No 831.

Zhang, Q., Turton, R., Bhattacharyya, D., 2016. Development of Model and Modelpredictive Control of an MEA-based Postcombustion $\mathrm{CO}_{2}$ Capture Process. Industrial and Engineering Chemistry Research, 55(5):1292-1308. doi:10.1021/acs.iecr.5b02243. 